\title{
Les croyances des futurs enseignants : est-il possible de les faire évoluer en cours de formation initiales et, si oui, comment?
}

Future teachers' beliefs and values: can these evolve during initial teacher training, and if so, how?

\section{Fanny Boraita et Marcel Crahay}

\section{(2) OpenEdition}

\section{Journals}

Édition électronique

URL : http://journals.openedition.org/rfp/4186

DOI : $10.4000 / \mathrm{rfp} .4186$

ISSN : 2105-2913

Éditeur

ENS Éditions

Édition imprimée

Date de publication : 16 décembre 2013

Pagination : 99-158

ISBN : 978-2-84788-494-4

ISSN : 0556-7807

\section{Référence électronique}

Fanny Boraita et Marcel Crahay, « Les croyances des futurs enseignants : est-il possible de les faire évoluer en cours de formation initiales et, si oui, comment? », Revue française de pédagogie [En ligne], 183 | avril-mai-juin, mis en ligne le 16 décembre 2016, consulté le 10 décembre 2020. URL : http:// journals.openedition.org/rfp/4186; DOI : https://doi.org/10.4000/rfp.4186 


\section{NOTE DE SYNTHÈSE}

\section{Les croyances des futurs enseignants : estili possible de les faire évoluer en cours de formation initiale et, si ovi, comment? Fanny Boraita et Marcel Crahay}

L'objet de cette note de synthèse est d'approfondir la question de l'évolution des croyances posée par Crahay, Wanlin, Issaieva et Laduron (2010) en s'interrogeant sur l'efficacité des programmes de formation initiale à faire évoluer les croyances des futurs enseignants. Cette question reste en effet controversée et il est malaisé de tirer une conclusion franche. Les raisons de cet état de fait sont plurielles. Il convient d'abord de relever que la notion même de changement n'est pas clairement définie. En outre, nonobstant cette lacune conceptuelle majeure, les chercheurs concluent souvent à des modifications de surface éphémères et parfois à des transformations substantielles et durables. Il faut en sus relever l'hétérogénéité des recherches menées dans ce champ : études longitudinales ou transversales, quantitatives ou qualitatives, sur un ensemble d'enseignants ou sur des cas isolés, certaines portant sur les croyances de façon générale et d'autres sur des croyances spécifiques. Dans cette note, il s'agit d'étudier l'effet de la formation initiale en tenant compte de cette variabilité.

Dans un premier temps, nous tâchons de recenser les études portant sur l'évolution des croyances des enseignants en formation initiale en tenant compte de leur cadre respectif. Ainsi, nous passons successivement en revue les effets de la formation initiale en nous focalisant sur les évaluations générales des dispositifs de formation pour ensuite examiner l'impact des cours théoriques, des programmes spécifiques et des stages pratiques. Afin d'aboutir à des conclusions aussi précises que possible, nous esquissons une taxonomie des changements possibles et dressons les cadres méthodologiques de chacune des études recensées. Nous nous intéressons ensuite aux solutions invoquées par les chercheurs pour pallier la résistance des croyances aux effets de la formation initiale. Dans la discussion finale, nous nous interrogeons sur l'adéquation des dispositifs de formation et, dans la foulée, esquissons des pistes à explorer pour favoriser le développement professionnel des futurs enseignants. Nous terminons par un retour sur la notion de croyance en posant la question cruciale à nos yeux : qu'est-ce qu'une "bonne » croyance?

Mots-clés (TESE) : savoir, enseignant stagiaire, formation des enseignants, développement des capacités professionnelles. 


\section{INTRODUCTION : À PROPOS DE LA MALLÉABILITÉ DES CROYANCES DES ENSEIGNANTS}

Dans une note de synthèse intitulée "Fonctions, structuration et évolution des croyances (et connaissances) des enseignants ", Crahay, Wanlin, Issaieva et alii (2010) posent la question suivante : les croyances et les connaissances des enseignants peuvent-elles évoluer? Se fondant sur une importante revue de la littérature, ils proposent une réponse positive mais nuancée à cette question. Ils notent que les chercheurs ne s'entendent pas sur ce qu'il faut considérer comme un changement dans les croyances. La conception la plus courante est celle de la substitution : la ou les croyance(s) B remplace(ent) la ou les croyance(s) A. Ils relèvent également que l'on peut distinguer des modifications de surface (first order change dans la littérature anglo-saxonne, voir notamment Cuban, 1988) et des transformations en profondeur (second order change ou structural changes). Ainsi, lorsque Lanier et Little (1986) concluent que changer le comportement et les pensées des enseignants est possible, ils parlent principalement de modifications de procédures d'enseignement et, de surcroît, de changements de premier ordre ou de surface. En revanche, lorsque Richardson et Placier (2001) reprennent la question quinze ans plus tard, ils la posent dans un autre contexte conceptuel et envisagent presque uniquement les transformations en profondeur. Entre la revue de Lanier et Little (1986) et celle de Richardson et Placier (2001), un changement de paradigme s'est opéré dans la recherche en éducation et, plus particulièrement, dans celle portant sur l'étude de l'enseignement et des enseignants. D'une part, le behaviorisme a laissé place au cognitivisme (Crahay, Wanlin, Issaieva et al., 2010) et, d'autre part, la conception de la professionnalité enseignante a été l'objet d'une sorte de révolution paradigmatique à laquelle Fenstermacher (1986) a beaucoup contribué. Pour ce philosophe de l'éducation, l'enseignant ne peut se contenter d'être un technicien mettant en œuvre des procédures efficaces ; il doit être un professionnel conscient de ses gestes pratiques et capable de les justifier, que ce soit en invoquant les recherches ou son expérience professionnelle. Par ailleurs, pour Fenstermacher (1986), si les résultats des recherches en éducation doivent nourrir la réflexion des enseignants, ceux-ci ne peuvent suffire à eux seuls à définir ce qu'est un « bon enseignement ». C'est dans ce cadrage conceptuel qu'il conviendrait idéalement de poser la question de savoir dans quelle mesure les croyances initiales des enseignants en formation évoluent et changent, notamment en se nourrissant de leurs connaissances des recherches. Malheureusement, la plupart des recherches portant sur les enseignants en formation n'assument pas pleinement ce positionnement théorique.

Dès lors que l'on envisage des transformations en profondeur dans les croyances des enseignants, rares sont les chercheurs qui les pensent possibles sur le court terme (cf. Crahay, Wanlin, Issaieva et al., 2010). Dans cette perspective, le point de vue défendu par Richardson et Placier (2001) nous interpelle : ces chercheurs concluent de leur large revue de la littérature que les croyances et connaissances des enseignants changent rarement suite à la formation initiale, mais évoluent chez les enseignants en fonction, soit de façon "spontanée ", c'est-à-dire indépendamment de tout processus de formation in service, soit lorsque ces formations s'inscrivent dans une approche de longue durée qui engage l'enseignant dans un processus de changement (Chin \& Benne, 1969). Cette conclusion doit être considérée avec sérieux vu la qualité de la recension de recherches à laquelle ont procédé ces chercheurs. Or, elle nous confronte à un paradoxe : ce serait en quelque sorte au moment où les enseignants sont censés construire des croyances professionnelles quant à l'enseignement et à l'apprentissage qu'ils le feraient le moins. Ce constat est étonnant car il conduit à s'interroger sur la pertinence et l'efficacité des dispositifs de 
formation initiale, dispositifs auxquels les pouvoirs publics consacrent d'importants financements. C'est cette question que nous souhaitons reprendre dans la présente note de synthèse ${ }^{1}$. Celle-ci s'inscrit dans la foulée de celle de Crahay, Wanlin, Issaieva et alii (2010) et reprend à son compte le positionnement conceptuel quant à la définition englobante de la notion de croyances².

Dans un premier temps, nous revisiterons la conclusion de Richardson et Placier (2001) selon laquelle les croyances des futurs enseignants (FE) résistent au changement pendant la formation initiale et, de ce fait, évoluent peu à ce moment-là. Cette conclusion nous paraît devoir être réexaminée eu égard à l'hétérogénéité des recherches menées sur cette problématique. Avec Crahay, Wanlin, Issaieva et alii (2010), nous pensons que Richardson et Placier (2001) n'ont pas suffisamment tenu compte de cette hétérogénéité. Pour nous, il convient de traiter les résultats selon leur cadre méthodologique respectif : type de mesures prises, échantillons, façon de concevoir le changement, etc. De plus, il faut relever que certaines études observent des croyances particulières (par exemple celles liées à l'enseignement des mathématiques) et d'autres des croyances plus générales (la façon de concevoir l'enseignement en général). Avec Crahay, Wanlin, Issaieva et alii (2010), nous reprochons à Richardson et Placier (2001) d'avoir mis toutes les recherches sur le même pied comme si les conclusions ne dépendaient pas de la méthodologie de recherche utilisée, ni du type de croyances considérées.

Dans un second temps, nous envisagerons les raisons invoquées par les chercheurs pour expliquer la résistance à la formation des croyances des FE, ce qui nous poussera à examiner les critiques qui ont été adressées aux dispositifs de formation. Dans la foulée, nous passerons en revue les solutions qui ont été envisagées au problème posé par l'apparente faible efficacité de la formation initiale quant au changement de croyances. Ceci nous amènera donc à discuter la question : comment envisager le changement de croyances des FE en cours de formation initiale?

Enfin, dans une dernière partie, nous esquisserons une discussion et proposerons des pistes de recherche pour le futur.

\section{LES EFFETS DE LA FORMATION INITIALE SUR LES CROYANCES DES FUTURS ENSEIGNANTS}

Selon des chercheurs comme Richardson (1994), Borko et Putnam (1996) et Richardson et Placier (2001), les croyances des FE évoluent peu au cours de leur formation initiale. C'est peu ou prou la conclusion qu'ils tirent de leurs revues des études qui se sont intéressées à l'influence de la formation sur les croyances des FE. Cette conclusion ne fait cependant pas l'unanimité parmi les chercheurs. En effet, à côté d'une majorité de résultats concluant à la résistance des croyances, certains chercheurs sont plus nuancés dans leurs conclusions. Bolin (1990) et Markel (1995) retiennent de leurs recherches que certains FE modifient leurs croyances. Civil (1993) montre quant à lui qu'après une phase de résistance, certains FE changent leurs croyances alors que, chez d'autres, celles-ci restent les mêmes. Plus encore, des études concluent à des changements pour certaines croyances et pas pour d'autres (Feiman-Nemser, McDiarmid, Melnick et al., 1989 ; Olson, 1993 ; Woolfolk Hoy \& Burke Spero, 2005). Cette variabilité nous pousse donc à observer plus finement les recherches menées dans le domaine. Pour ce faire, en nous basant 
sur les apports de Shapiro (1991), Crahay, Wanlin, Issaieva et alii (2010) et Kagan (1992), nous proposons de distinguer divers types de changement ou d'évolution:

- évolution personnelle : les croyances des FE évoluent dans le sens d'un enrichissement car ils acquièrent et/ou développent de nouvelles connaissances sur eux-mêmes en tant qu'enseignants ;

- évolution managériale : les croyances des FE évoluent dans le sens d'un enrichissement car ils acquièrent et/ou développent de nouvelles connaissances managériales sur le processus d'enseignement-apprentissage ;

- changement de croyances : les croyances des FE ont changé, avant ils pensaient $A$ et maintenant ils pensent $B$ (cf. le processus de substitution évoquée par Crahay, Wanlin, Issaieva et al., 2010) ;

- renforcement de croyances initiales : les croyances initiales des FE se voient renforcées.

Il faut, parallèlement, noter les cas de statu quo, quel que soit le type de changement visé : les croyances des FE ne se modifient d'aucune façon pendant le processus de formation. On note également des phénomènes de retour en arrière ou d'effet " à rebours " : avant les FE pensaient $A$, puis ils ont pensé $B$ et maintenant ils pensent à nouveau $A$. Cette façon de catégoriser les résultats des recherches reste à la superficie des phénomènes qui sous-tendent probablement ces évolutions et changements. Ceux-ci reflètent - supposons-nous - des apprentissages et/ou des processus de développement professionnel, voire identitaire. Par souci de rigueur, nous en restons à cette façon de décrire les résultats des recherches, laissant à des recherches futures le soin de préciser les processus psychologiques qui sont sous-jacents à ces évolutions et changements ; dans l'état actuel, il ne nous paraît pas possible d'aller plus en profondeur sans verser dans la supputation.

Le tableau 1 en annexe présente les 44 études passées en revue dans cette partie. Celles-ci ont été retenues d'une part au terme de la consultation des articles de la bibliographie de Richardson et Placier (2001) "Teacher Change » issu du Handbook of research on teaching et, d'autre part, en consultant les articles datant de 1995 à nos jours dans plusieurs revues, à savoir majoritairement Journal of Teacher Education, Teaching and Teacher Education, La Revue française de pédagogie, La Revue suisse des sciences de l'éducation, La Revue québécoise des sciences de l'éducation. Pour ce faire, nous avons utilisé les mots-clés suivants : croyance, futur enseignant, enseignant en formation, formation initiale, belief, conception, representation, preservice teacher, student teacher, initial training. Bien que nous ayons exploré plusieurs revues francophones, force est de constater que ce champ thématique - les effets de la formation initiale sur les croyances des FE - est fort peu exploré par les chercheurs de langue française. Ce constat est - sans doute en partie - explicable par l'usage du terme " croyances ", peu prisé par les chercheurs francophones en sciences de l'éducation.

Les différentes recherches retenues ont été classées selon le type de formation envisagée (formation au sens large, cours théoriques, etc.), mais aussi en fonction du type de croyances concernées. À cet égard, nous avons distingué quatre catégories (voir tableau 1 en annexe) :

- le processus d'enseignement-apprentissage, soit de façon générale, soit selon qu'il concerne plus spécifiquement :

a. le style et/ou la façon d'enseigner ainsi que le processus d'apprentissage des élèves ;

b. les rôles respectifs de l'enseignant et des élèves au sein du processus et leur relation ;

c. l'enseignement des mathématiques ;

d. l'enseignement de la lecture ;

e. l'enseignement de l'écriture ; 
- la recherche : dans le domaine de l'éducation, la façon dont elle est perçue et considérée ;

- l'évaluation : sa forme, ses fonctions et sa mise en place ;

- la pratique du redoublement : ses effets sur les élèves et leurs résultats.

Ces catégories sont hétérogènes, mais elles reflètent l'état du champ ; les formateurs et les chercheurs ont étudié la catégorie de croyances correspondant à leurs intérêts propres : les mathématiques ou la lecture pour ceux qui sont experts de l'un ou l'autre de ces domaines, l'évaluation pour ceux qui tentent de modifier les habitudes dans ce domaine, le redoublement pour ceux qui luttent contre l'échec scolaire, etc.

Notons également la diversité des contextes, des institutions et des disciplines concernées. Ceci est probablement le fruit, pour une part, de la diversité des dispositifs de formation de par le monde et, d'autre part, du manque d'unification du champ de la recherche portant sur les effets de la formation initiale des enseignants. Comme discuté par Crahay, Wanlin, Issaieva et alii (2010), il n'est pas garanti que la nature des croyances dans ces différents domaines soit de même nature. Ainsi, les débats sont bien plus vifs à propos de la lecture et du redoublement qu'à propos des mathématiques ou de l'écriture. II faut constater, hélas, que cette question est fort peu investiguée par les chercheurs.

Dans cette partie, nous présenterons donc les études ayant mesuré les effets de la formation initiale sur les croyances des FE en synthétisant leurs résultats selon la taxonomie décrite ci-dessus. Dans un premier temps, nous aborderons les effets de la formation dans sa globalité. Ensuite, nous distinguerons les effets des cours théoriques et des stages pratiques. Nous aborderons également les effets de cours spécifiquement conçus pour provoquer un changement de croyances et l'impact de la pratique enseignante chez les enseignants en formation.

\section{Les effets des programmes de formation}

Nous avons recensé 14 études portant sur les effets des programmes de formation eu égard à l'évolution des croyances. Dix d'entre elles ont trait aux croyances relatives au processus d'enseignement, dont certaines portent sur des croyances relatives à des disciplines spécifiques et, plus particulièrement, les mathématiques (4 études) et la lecture (1 étude). Deux études s'intéressent respectivement à la place de l'enseignant et à celle des élèves dans le processus d'enseignement, une étude porte sur les conceptions de l'évaluation et une autre sur celles concernant la pratique du redoublement. Le tableau 2 en annexe donne une vue synthétique mais plus précise des recherches passées en revue ci-dessous.

Plusieurs études ont mis en évidence des changements dans les croyances des FE au fil de leur formation. C'est notamment le cas de Markel (1995) qui a observé et interviewé $5 \mathrm{FE}$ primaires lors de leur participation à des séminaires de cours et qui constate des changements chez chacun d'eux, ainsi que de Mattheoudakis (2007) qui montre que les croyances de 66 FE secondaires interrogés via des questionnaires évoluent année après année au fil de la formation. Lorsque cette chercheuse compare les croyances des FE au début et à la fin de leur formation, elle constate même de réels changements pour la majorité d'entre eux. C'est le cas également de Hollingsworth (1989) qui a observé 14 FE primaires et secondaires durant 5 ans en combinant l'utilisation de questionnaires avec des interviews et des observations. La chercheuse remarque qu'à l'entrée en formation les étudiants considèrent que les élèves sont peu impliqués dans le processus d'apprentissage de la lecture, c'est plutôt grâce aux instructions du professeur que leur apprentissage 
s'accomplit. À la fin de la formation, elle constate que les FE pensent que les élèves construisent activement leurs propres connaissances. De plus, ceux qui avaient une vision globale de l'enseignement de la lecture en début de formation évoluent vers une compréhension plus fine du processus spécifique de l'apprentissage des élèves. Hoy et Rees (1977) montrent quant à eux qu'au fil de la formation les FE secondaires qu'ils ont interrogés à partir de questionnaires se focalisent davantage sur les élèves et leur processus d'apprentissage, alors qu'en début de formation, ils ne s'intéressent qu'à la gestion de la classe. Les résultats de la recherche de $\mathrm{Ng}$, Nicholas et Williams (2010) vont également dans ce sens. Les croyances des $115 \mathrm{FE}$ secondaires ayant répondu aux questionnaires quant au rôle de l'enseignant et à sa place au sein du processus d'enseignement-apprentissage changent pendant la formation. Au début, ils le considèrent comme le maître de la classe, celui qui doit gérer l'ordre et la discipline alors qu'à la fin des cours, ils le conçoivent comme étant avant tout en relation avec ses élèves. McDiarmid (1990) a aussi constaté des changements chez ses propres étudiants qui se destinent à l'enseignement des mathématiques. En combinant l'utilisation de questionnaires avec des interviews et des observations, le chercheur remarque qu'au fil de la formation, les FE prennent petit à petit conscience qu'il y a plusieurs façons d'enseigner un même concept et qu'il ne suffit pas de connaître un sujet pour pouvoir l'enseigner alors qu'ils n'en ont pas conscience au début de la formation. En revanche, dans le cadre d'études de cas portant respectivement sur 1 et 2 FE primaires, Bolin (1990) et Olson (1993), qui recourent tous deux à l'observation et aux interviews, concluent à une résistance au changement : les conceptions de FE relatives à l'enseignement ne changent pas.

Plusieurs études s'inscrivent dans le cadre du programme TELT (Teacher Education and Learning to Teach). Ce programme s'est intéressé aux changements dans les conceptions de l'enseignement des mathématiques et de l'écriture de 700 enseignants en formation et en fonction, à travers une étude longitudinale portant sur les effets de 11 programmes de formation. Des questionnaires leur ont été soumis à leur entrée en formation et à divers moments en cours de formation, un sous-groupe de FE a également été interviewé de façon plus intensive. Au fil des années, les FE ont été réinterrogés. Borko et Putnam (1996) ont recensé les résultats du programme TELT et débouchent sur des résultats plutôt mitigés : les conceptions de certains FE évoluent en cours de formation, ceux-ci approfondissant leurs connaissances des contenus; cependant, d'autres FE traversent leur formation en voyant toujours les mathématiques comme un ensemble de règles et de signes abstraits, sans comprendre leurs réels liens et significations (Wilcox, Schram, Lappan et al., 1991). Au final, aux yeux de Borko et Putnam (1996) mais aussi de Wilcox, Schram, Lappan et alii (1991) qui se sont concentrés sur l'observation de 23 FE primaires ayant également répondu à un questionnaire, les changements observés restent trop superficiels pour conclure à un réel changement. Toujours concernant le programme TELT, Civil (1993) ne remarque pas, lui non plus, de changement de croyances parmi les 8 FE primaires qu'il a observés et interviewés par rapport à leurs conceptions de l'enseignement des mathématiques. Les FE semblent toujours vouloir enseigner les mathématiques de la même façon qu'ils les ont eux-mêmes apprises lorsqu'ils étaient élèves, et ce même si la plupart d'entre eux ont eu des difficultés à apprendre les mathématiques à l'école.

Bramald, Hardman et Leat (1995) ont comparé les conceptions que détenaient 162 FE secondaires de plusieurs disciplines au début et à la fin de leur première année de formation en recourant à la fois à la passation de questionnaires et à la réalisation d'interviews. En début d'année, ils ont tous une conception assez traditionnelle de l'enseignement : l'enseignant est au centre du processus d'apprentissage. En fin d'année, les FE destinés à l'enseignement de l'anglais et de la géographie ont 
une vision du processus d'enseignement selon laquelle l'élève est au centre des apprentissages. En revanche, les autres FE conservent leur représentation initiale.

Graham (2005) s'est intéressé à l'impact de la première année de formation sur les conceptions de l'évaluation en combinant l'utilisation de questionnaires, d'observations et d'interviews. En entrant en formation, les 38 FE secondaires concernés par l'étude voient l'évaluation comme quelque chose qui arrive à la fin d'une séquence ou de l'année scolaire, privilégiant une perspective certificative de l'évaluation. Au terme de leur première année de formation, les FE considèrent que l'évaluation peut se faire au quotidien et sous de multiples formes. Pour eux, les évaluations servent désormais à obtenir des informations sur les progrès des élèves et pas seulement à leur mettre une note. Les FE attribuent principalement ces changements dans leurs conceptions de l'évaluation aux textes théoriques distribués lors des cours et des débats sur ceux-ci avec leurs professeurs et les autres FE. Ceci témoigne du rôle joué par les connaissances dans l'évolution et la modification des croyances.

Crahay, Ory et Boraita (2012) ont étudié l'évolution des croyances de $650 \mathrm{FE}$ primaires sur le redoublement. Ces chercheurs ont interrogé à deux reprises (chaque fois en fin d'année scolaire à l'aide d'un questionnaire) plusieurs cohortes de FE se situant à différents points du cursus de formation. Leurs résultats montrent qu'en tout début de formation, les étudiants sont totalement « pour » la pratique du redoublement alors que ceux de deuxième et de dernière année ont des avis plus mitigés. En fait, il apparaît que les croyances des FE convergent de plus en plus en cours de formation avec les croyances de leur formateur. Cependant, ces changements paraissent superficiels dans la mesure où les FE n'expriment guère de modifications sur les raisons pour lesquelles ils finissent par juger le redoublement inefficace.

Parmi les 14 études recensées ci-dessus, on observe autant de changements que de statu quo dans les résultats; plus précisément, 8 recherches concluent à des changements face à 6 recherches qui n'en montrent pas. Ce sont les croyances relatives à l'enseignement au sens large qui sont majoritairement investiguées. Parmi les croyances qui subissent des changements, on trouve donc celles sur l'enseignement, mais également celles concernant le rôle de l'enseignant et celui des élèves, ainsi que les conceptions de l'évaluation. De plus, les changements établis renvoient essentiellement au processus de substitution défini par Crahay, Wanlin, Issaieva et alii (2010).

La difficulté de conclure quant à l'effet des programmes de formation vient de ce que les auteurs fournissent peu d'indications sur les caractéristiques de ces programmes. Seuls Mattheoudakis (2007) et Graham (2005) précisent l'importance des apports théoriques et leur rôle dans l'évolution et le changement des conceptions des FE. Globalement, on sait donc peu de choses sur les moyens mis en œuvre pour faire évoluer et changer les croyances sur lesquelles les FE sont interrogés. De plus, le nombre de FE auquel s'intéressent les études mises en évidence varie d'un seul étudiant à plus de 700 , et les outils pour récolter les données relèvent autant de méthodes qualitatives que quantitatives. De façon générale, parmi les études recensées ci-dessus, ce sont davantage les recherches quantitatives menées sur un échantillon assez conséquent de FE qui semblent fournir des résultats positifs en termes de changements de croyances au cours de la formation initiale. Nous reviendrons plus en détail sur la variabilité des échantillons et des méthodes utilisées pour récolter les données par la suite. 


\section{Les effets des cours théoriques}

Nous avons recensé 10 études dans lesquelles les chercheurs examinent les effets de cours théoriques sur les croyances des FE. Ces cours sont majoritairement dispensés en début de formation et servent plutôt d'introduction aux différents courants pédagogiques ou aux sciences de l'éducation. Seules deux études font exceptions : celle de Cabaroglu et Roberts (2000) porte sur les effets d'un cours de langues étrangères et celle de Holt-Reynolds (1992) a trait à l'enseignement de la lecture. Les croyances auxquelles les chercheurs de ces études se sont intéressés sont essentiellement celles relatives au processus d'enseignement. Seule une d'entre elles concerne la recherche en éducation. Le tableau 3 en annexe présente les 10 études auxquelles nous nous sommes intéressés.

Trois de ces dix études concluent à des effets positifs ou mitigés des cours théoriques. C'est le cas notamment de l'étude de Feiman-Nemser, McDiarmid, Melnick et alii (1989) qui combine plusieurs méthodes (la passation de questionnaires, la réalisation d'interviews et l'observation) et qui met en évidence une évolution dans les conceptions de 91 FE primaires entre le début et la fin d'un cours d'Introduction aux sciences de l'éducation, cours dispensé lors de la première année de formation. Au début, les FE conçoivent l'enseignement comme le simple fait que « l'enseignant enseigne » et que les élèves apprennent, alors qu'à la fin du cours, ils ont pris conscience qu'enseigner est un processus beaucoup plus complexe et ils distinguent les concepts d'enseignement et d'apprentissage. Ils abordent également le contexte de la classe et le niveau que les élèves doivent atteindre à l'issue de l'enseignement donné.

D'autres études fournissent des résultats plutôt mitigés. C'est le cas notamment de l'étude de Cabaroglu et Roberts (2000) qui ont interviewé 20 FE secondaires pendant leur cours de Langues modernes étrangères. Leurs résultats montrent des évolutions pour 17 d'entre eux; celles-ci concernent les croyances relatives à l'enseignement et à l'apprentissage. Ils observent également des changements pour 2 FE mais, pour 1 seul FE, aucune modification n'apparaît. Rappelons que tous les " formés " ont été soumis au même dispositif. Tanase et Wang (2010) ont suivi 4 FE primaires tout au long du cours de Méthodes en éducation en les interrogeant via des questionnaires et en analysant leurs différentes productions (portfolio, notes). Seuls 2 d'entre eux changent leurs croyances initiales : au début du cours, ils considèrent l'enseignement comme une transmission de connaissances par un expert-enseignant, l'élève y est passif et reçoit simplement l'information, alors qu'à la fin du cours ils conçoivent l'enseignement comme un processus d'interactions entre l'élève et l'enseignant ou entre les élèves eux-mêmes. L'organisation de la classe et le climat sont également perçus différemment par ces deux FE : d'un climat plutôt strict dans lequel chaque élève est assis et écoute en silence, les FE se le représentent comme un lieu d'échanges et d'interactions.

À l'opposé, plusieurs autres études ne montrent quant à elles pas de changement dans les croyances. McDiarmid (1992) conclut de son étude par questionnaires et interviews que, même à la suite d'une séquence de cours portant spécifiquement sur l'Éducation multiculturelle et proposant aux FE une série d'exposés sur le sujet, leurs conceptions changent vraiment peu. McDaniel (1991, cité dans Kagan, 1992) a examiné dans quelle mesure $3 \mathrm{FE}$ primaires et $19 \mathrm{FE}$ secondaires intégraient et assimilaient le sens des contenus d'un cours de Philosophie et histoire de l'éducation. Ses résultats issus d'observations et d'interviews montrent qu'aucun des contenus de cours ne modifie leurs conceptions initiales. Les FE ont plutôt tendance à retirer du cours les contenus qu'ils peuvent lier à leurs propres croyances et à leur vécu personnel de la classe. Book, Byers et Freeman (1983) aboutissent à une 
conclusion similaire en n'utilisant que la technique du questionnaire : les cours de Psychologie de l'éducation et ceux sur les Fondements socio-philosophiques de l'éducation n'affectent pas les conceptions des 473 FE primaires. II en va de même de l'étude de Hoy et Woolfolk (1990) qui montre que peu importe le cours suivi, les 191 FE primaires du cours de Méthodes en éducation et de celui de Psychologie du développement, qui ont été observés et interrogés via des questionnaires, conservent leurs conceptions initiales. Ils restent très confiants dans leur capacité à motiver les élèves et à surmonter les différences socioculturelles au sein du groupe-classe et, ce qui compte pour eux, c'est d'abord le contrôle de la classe et la gestion des problèmes entre les élèves. De même, Gitlin, Barlow, Burbank et alii (1999) n'observent aucun changement dans les conceptions sur la recherche en éducation de 37 FE (dont 17 primaires et 20 secondaires), celles-ci étant mesurées à l'aide de questionnaires et d'interviews, au début et à la fin d'un cours typiquement focalisé sur cette thématique. En effet, les FE attribuent aux recherches les mêmes limites qu'au début du cours ; pour eux, elles ne sont ni accessibles ni objectives car qu'elles ne tiennent pas compte de la réalité. Et c'est encore une conclusion analogue que tire Holt-Reynolds (1992) qui a observé l'évolution des conceptions de $191 \mathrm{FE}$ en les invitant à répondre à un questionnaire et en les interviewant lors d'un cours de Méthodologie de l'enseignement de la lecture. Ses résultats montrent que les FE considèrent toujours l'enseignant comme l'élément central du processus d'enseignement bien que le cours soit focalisé sur l'élève. Holt-Reynolds (1992) étaye ses résultats en concluant que les conceptions personnelles des FE quant au rôle de l'enseignant et leurs images des leçons idéales sont plus fortes et déterminantes que les conceptions enseignées lors de la formation. Les croyances initiales se révèlent donc bien être le ciment des conceptions des FE et de leur éventuelle évolution. Une idée qui est appuyée par Feiman-Nemser et Buchmann (1989) qui, au terme d'une étude portant sur les observations et interviews de 6 FE primaires, concluent que leurs croyances initiales en matière d'enseignement se voient renforcées par les cours théoriques qu'ils ont reçus.

En définitive, la tendance qui se dégage de ces recherches est assez nette: les cours théoriques affectent fort peu les croyances personnelles des FE. Bien plus, deux études (celle de Feiman-Nemser et Buchmann et celle de McDaniel) montrent que les croyances initiales se voient renforcées par les enseignements théoriques. II convient, toutefois, de rester prudent tant les études diffèrent par leurs échantillons, qui varient de 4 à $473 \mathrm{FE}$, et par leurs méthodologies; celles-ci vont de l'usage du questionnaire à la combinaison d'observations, de prises de notes, de portfolios, de questionnaires avant et après le cours chez Tanase et Wang (2010). II faut également souligner le caractère global des conclusions tirées par les chercheurs. On constate cependant que les recherches les plus récentes sont nettement plus nuancées. Ainsi, Cabaroglu et Roberts (2000) d'une part, et Tanase et Wang (2010) d'autre part fournissent les nombres de FE qui progressent et de ceux qui ne le font pas. Il est regrettable que les recherches précédentes ne fournissent pas ce type d'information. Notons encore que, dans le cas de ces deux études, on n'a pas d'information concernant les élèves qui changent leurs croyances et ceux qui ne le font pas. Enfin, on regrettera le peu d'informations que donnent ces recherches concernant les cours théoriques évalués.

\section{Les effets de programmes spécifiquement conçus pour changer les croyances}

Nous avons recensé 6 recherches dans lesquelles des programmes spécifiques sont mis en place pour faire changer les croyances des FE. Cinq d'entre elles s'intéressent aux croyances relatives à l'enseignement, dont deux concernent plus 
spécifiquement l'enseignement des mathématiques. Seule une étude s'est focalisée sur les conceptions des FE eu égard aux effets de la pratique du redoublement. Le tableau 4 en annexe présente une brève synthèse de ces recherches.

Tillema et Knol (1997) ont formulé l'hypothèse que les programmes de formation peuvent avoir des effets substantiels sur les croyances de FE à condition de prendre en considération leurs croyances initiales et de les impliquer dans la construction de nouvelles structures de connaissances. Dans une étude de type quasi expérimental, ces chercheurs ont comparé les effets de deux programmes de formation sur les croyances de 153 FE primaires:

- le Conceptual Change Program, qui favorise l'apprentissage auto-régulatif et qui considère le changement des croyances comme le moteur majeur du développement des compétences ; dans ce programme, 85 FE sont encouragés à expliciter leurs croyances et à les mettre en lien avec les nouvelles perspectives pédagogiques exposées lors des cours;

- le Direct Instruction Program, plus classique, qui consiste en la transmission des connaissances et en leur application par $68 \mathrm{FE}$.

Peu importe le programme suivi, les résultats ne montrent pas de changements conséquents dans les croyances des FE. Néanmoins, pour Tillema et Knol (1997), le Conceptual Change Program peut produire des changements superficiels comparativement au Direct Instruction Program qui n'en apporte aucun.

Plusieurs chercheurs ont tenté de moduler leurs programmes de formation en changeant leurs méthodes ou en insérant des séquences spécifiques dans le but de provoquer une évolution dans les croyances des FE. Bullough et Stokes (1994) ont mis en place une séquence de travail intensif basé sur l'utilisation des métaphores de leurs étudiants. Au début de la séquence, les 22 FE secondaires construisent des métaphores essentiellement empreintes de leurs expériences personnelles vécues avec des enfants et/ou des adolescents. Celles-ci sont parfois fort naïves ; par exemple, certains $\mathrm{FE}$ se représentent l'enseignant comme un père ou une mère, comme un transmetteur de savoir pur et dur. À la fin de la séquence, les chercheurs remarquent que la naïveté a laissé place à une certaine maturité. Les FE abordent les préoccupations des enseignants, leur fonctionnement en classe et avec les élèves. Bullough et Stokes (1994) ne concluent pas à un réel changement mais plutôt à une évolution subtile : les croyances des FE ne changent pas mais elles évoluent vers un niveau plus complexe, plus sophistiqué, moins traditionnel. À travers l'analyse de toutes les métaphores récoltées, Bullough et Stokes (1994) observent une " perte de l'innocence " qui succéderait à " l'optimisme irréaliste " dont font preuve les FE (Weinstein, 1989).

Calderhead et Robson (1991) ont suivi intensivement 12 FE primaires durant leur première année de formation. Les FE ont été interrogés à plusieurs reprises sur leurs craintes, leurs images d'eux-mêmes en tant qu'enseignants et leurs conceptions de l'apprentissage des élèves. Au début de la formation, les conceptions qu'ils ont du bon enseignement sont construites sur la base de leurs expériences de classe en tant qu'élèves. Ces images se rapportent uniquement à un ou deux modèles d'enseignement et ne semblent pas être flexibles pour d'autres contextes de classe. Calderhead et Robson (1991) ont mis en place des tâches expérimentales étalées sur toute l'année. Lors de certains cours de la formation, les FE avaient l'occasion de visionner des vidéos qui montraient des enseignants dispensant des leçons de mathématiques ou d'écriture selon différents styles. Ils étaient alors invités à commenter et critiquer ces vidéos et à imaginer comment eux pourraient enseigner une matière similaire. À la fin de l'année de formation, Calderhead et Robson (1991) ont demandé à chaque étudiant de créer une préparation de leçon 
qu'il pourrait enseigner de façon très détaillée, notamment avec les dialogues entre l'enseignant et les élèves. Malgré les tâches expérimentales insérées dans le programme de formation, les résultats des chercheurs montrent que les FE se concentrent uniquement sur leurs propres actions d'enseignant plutôt que sur celles des élèves. Ils semblent être incapables d'adapter leurs images de l'enseignant et celles des leçons à des situations différentes et à des besoins d'élèves différents.

D'autres chercheurs ont modulé leurs propres cours de façon à expliciter et à remettre en question les croyances des FE. C'est le cas de Ball (1988) qui montre une évolution dans les conceptions de ses 25 FE primaires quant à l'enseignement des permutations en mathématiques. Levin et Ammon (1992) constatent aussi une évolution dans les conceptions de 4 FE primaires suivant un programme de formation conçu selon une perspective piagétienne de l'apprentissage et du développement. Ces chercheurs démontrent que les croyances des FE peuvent changer et évoluer lors de la formation initiale si celle-ci se penche spécifiquement sur cet objectif de changement de conceptions.

Nicolas (2002) a mené une étude quasi expérimentale en Belgique dans laquelle elle a suivi l'évolution des croyances relatives au redoublement de 36 FE primaires, dont la moitié (groupe expérimental [GE]) a bénéficié d'une séquence d'activités dont l'objectif est d'amener à réfléchir sur la pertinence de cette pratique, à laquelle la majorité des enseignants belges recourent et ceci malgré le fait que de nombreuses recherches ont montré son inefficacité (Crahay, 2004). Cette chercheuse observe un changement de croyances dans le sens souhaité pour 6 FE sur les 18 du GE, mais constate également l'évolution en sens contraire de 2 des FE de ce même groupe. Deux FE du groupe de contrôle (GC) évoluent dans le sens souhaité par la chercheuse. Par ailleurs, certains FE étaient convaincus de l'inefficacité du redoublement dès le début de la formation - 8 dans le GE et 3 dans le GC - et ne pouvaient donc que stabiliser leurs croyances en regard de l'orientation de la formation. D'autres FE (2 dans le GE et 4 dans le GC) étaient sans opinion claire en début de formation.

Quatre des 6 études recensées montrent des changements dans les croyances des FE, dont trois (Ball, 1988 ; Bullough \& Stokes, 1994 ; Levin \& Ammon, 1992) considèrent les changements de croyances de leurs FE quant à l'enseignement en terme d'évolution. Nicolas (2002), quant à elle, relate ses résultats en termes de substitution de conceptions eu égard aux effets du redoublement. Elle note de tels changements chez $10 \mathrm{FE}$ mais constate que, pour $2 \mathrm{FE}$, cette modification de croyances ne s'est pas opérée dans le sens souhaité par le programme mis en place par elle-même. Il est important de souligner pour la réflexion qui sera menée dans la deuxième partie de cet article que, dans ces quatre études, la formation met en œuvre des démarches basées sur l'explicitation et le travail des croyances initiales des FE. Comme nous y reviendrons plus loin, cette méthode de formation s'inscrit dans le paradigme de la dissonance cognitive (Festinger, 1957). Clairement, l'étude de Calderhead et Robson (1991) s'inscrit dans un autre courant de recherche, celui du Teacher planning : ces chercheurs ont demandé aux FE de préparer une leçon qu'ils ont filmée avant d'en débattre après visionnement en groupe-classe ${ }^{3}$. Les statu quo constatés par ces chercheurs ne vont pas à l'encontre de la conclusion positive que nous tirons de l'examen des études de Bullough et Stokes (1994), Ball (1988), Levin et Ammon (1992) et Nicolas (2002). En revanche, les conclusions mitigées tirées par Tillema et Knol (1997) à propos du programme Conceptual Change contrarient notre argumentation. Peut-être les résultats mineurs de Tillema et Knol (1997) sont-ils imputables à la méthode de mesure des effets utilisée. En effet, ces chercheurs ont utilisé des grilles d'observation en plus de questionnaires et, en définitive, évaluent autant les changements de pratiques que 
de croyances des FE. Par ailleurs, Bullough et Stokes (1994), qui observent des évolutions de croyances chez les FE de leur étude, ont filmé les FE pendant qu'ils enseignent et, donc, prennent aussi en considération les pratiques. Bref, vu le bémol apporté par l'étude de Tillema et Knol (1997), il est délicat de conclure de façon péremptoire. D'autres études en relation avec la discussion ouverte ici mériteraient d'être menées. Soulignons le fait que nous disposons de seulement 6 études pour mener cette discussion.

\section{Les effets de la formation pratique, des stages et de l'entrée en fonction}

Les étudiants en formation enseignante attendent beaucoup de l'apprentissage sur le terrain. Ils y prêtent une attention particulière, lui accordant une importance supérieure aux cours théoriques (Crahay, Wanlin, Issaieva et al., 2010). Tant les FE que les enseignants expérimentés affirment que l'expérience pratique est l'élément crucial de la formation des enseignants (Book, Byers \& Freeman, 1983 ; Lortie, 1975). Généralement, les images que les FE ont d'eux-mêmes en tant qu'enseignants et leurs conceptions de l'enseignement peuvent être modifiées par les premières expériences de terrain (Johnston, 1992). Nous avons recensé 18 études qui témoignent des effets de l'expérience de terrain. À cet égard, on peut distinguer quatre catégories d'études en fonction de la variable indépendante considérée :

- l'expérience pratique $4: 2$ études, et celles-ci concluent à des changements ;

- le premier stage : 8 études, dont 6 concluent à des changements ;

- les stages de façon générale : 6 études, dont 3 concluent à des changements ;

- l'entrée en fonction : 2 études, dont 1 conclut à des changements.

Parmi ces 18 études, 12 s'intéressent aux croyances concernant l'enseignement de façon générale, 2 sont relatives à la place des élèves et 3 à la place de l'enseignant ; seule une étude s'est penchée sur les croyances eu égard à la pratique du redoublement. Le tableau 5 en annexe présente une synthèse de l'ensemble de ces études et de leurs résultats.

\section{Effet de l'expérience pratique}

McDermott, Gormley, Rothenberg et alii (1995) ont suivi 2 groupes de FE primaires avant et après un stage en utilisant la technique des questionnaires. Le premier groupe de 45 FE n'avait encore jamais bénéficié d'expérience pratique et entrait donc sur le terrain pour la première fois alors que les 63 FE du deuxième groupe s'apprêtaient à entamer leur deuxième expérience pratique car ils avaient déjà bénéficié d'une expérience sur le terrain. Les résultats des chercheurs montrent qu'avant le stage, les deux groupes considéraient de la même façon l'enseignement, à savoir l'habileté à enseigner et à gérer une classe, alors qu'après le stage, les FE du premier groupe sont plus focalisés sur eux-mêmes et leur identité d'enseignant, et ceux du deuxième groupe plus concernés par l'apprentissage des élèves. McDermott, Gormley, Rothenberg et alii (1995) concluent de leur étude que les stages permettraient aux FE d'évoluer graduellement en acquérant d'abord des connaissances sur eux-mêmes en tant qu'enseignants et puis des connaissances plus centrées sur les élèves et le processus d'enseignement-apprentissage. Dans cette perspective, c'est avec des moments de terrain suffisants en formation initiale que les FE pourraient évoluer.

Leavy, McSorley et Boté (2007), qui ont également mené une recherche de type quasi expérimental sur $124 \mathrm{FE}$ primaires dont la moitié avait déjà été sur le terrain alors que l'autre n'avait été impliquée que dans une séquence de cours théoriques et didactiques, concluent de façon bien différente. Tous les FE de leur recherche 
ont été invités à livrer leurs métaphores sur leurs conceptions de l'enseignement et de l'apprentissage, avant et après le traitement (stages pour certains ; cours pour d'autres). Avant l'expérimentation, les métaphores béhavioristes étaient majoritaires dans les deux groupes, mais, après le traitement, les FE ayant été confrontés au terrain formulaient toujours majoritairement des métaphores béhavioristes, contrairement à ceux qui avaient suivi des cours théoriques et qui formulaient majoritairement des métaphores constructivistes de l'enseignement-apprentissage. Les chercheurs concluent de leur étude que l'expérience de terrain « effacerait » les acquis de la formation en termes de conceptions. Notons que cette interprétation de Leavy, McSorley et Boté (2007) est très discutable. Crahay, Wanlin, Issaieva et alii (2010) en proposent une autre. Ces chercheurs soulignent le jugement de valeur sous-jacent à la façon dont Leavy, McSorley et Boté (2007) lisent leurs résultats de recherche. Pour eux, il va de soi que les évolutions positives prennent la direction du socioconstructivisme. Ne remettant nullement en cause ce postulat, les chercheurs incriminent le processus de formation et, plus spécifiquement, le manque d'opportunités de réflexion quant à la façon dont les concepts enseignés sont liés aux réalités des classes. Cette hypothèse est recevable, écrivent Crahay, Wanlin, Issaieva et alii (2010), mais il est possible d'en proposer une autre. On peut en effet considérer que, " confrontés aux difficultés inhérentes à toute gestion de classe, les jeunes enseignants expérimenteraient l'aspect pratique des principes béhavioristes ainsi que leurs effets à court terme et, partant, les intégreraient à leur pratique quotidienne " (Crahay, Wanlin, Issaieva et al., 2010, p. 112). Car, ajoutent encore ces auteurs, il faut reconnaître que l'on ne dispose pas de preuves empiriques quant à la supériorité du socioconstructivisme par rapport au béhaviorisme en termes de gestion de la classe.

\section{Le premier stage}

De nombreux chercheurs (par exemple Bullough, Knowles \& Crow, 1991) constatent que, lorsque les enseignants entrent pour la première fois sur le terrain, ils ont peu de connaissances sur les élèves et sur la façon dont on enseigne. Ces connaissances et ces images évoluent et se peaufinent quand ils entrent en classe et qu'ils sont en interaction avec les élèves. La plupart des études qui ont investigué les effets du premier stage concluent d'ailleurs à des changements de conceptions. Ainsi, FlorioRuane et Lensmire (1990), qui ont observé et interviewé 6 FE primaires dispensant leurs premières leçons d'écriture, observent des substitutions de croyances entre le début et la fin du stage. Avant d'entrer sur le terrain, les FE se représentent l'enseignant comme la personne qui donne l'information aux élèves et qui leur enseigne les règles et codes de l'écriture. Au fil de leur stage, ces conceptions se sont modifiées et restructurées grâce à l'observation des élèves et à leurs nombreuses interactions avec eux. En se penchant sur la façon dont les élèves apprennent l'écriture, les FE ont pu prendre du recul sur leurs premières conceptions et reconnaître celles qui étaient incomplètes ou inexactes pour les reconstruire de façon plus appropriée. Borko et Putnam (1996) constatent également que c'est sur le terrain que les enseignants approfondissent leurs connaissances du processus d'enseignement-apprentissage et des rôles de l'enseignant et des élèves. Les 228 FE primaires et secondaires de l'étude combinant questionnaires et interviews de Cheng, Chan, Tang et alii (2009) et les 162 FE secondaires ayant répondu au questionnaire de Bramald, Hardman et Leat (1995) affirment que c'est l'expérience de terrain qui a modelé leurs conceptions de l'enseignement et de l'apprentissage. Avant leur premier stage, ces FE considèrent l'enseignement de façon transmissive alors qu'après, ils le conçoivent de façon plus dynamique avec un élève actif. Les $27 \mathrm{FE}$ primaires observés par Calderhead (1988) via plusieurs techniques (questionnaires, observations et interviews) rapportent aussi avoir beaucoup appris sur les élèves durant leur premier stage. En les observant et en interagissant avec eux, ils ont 
pu découvrir leurs comportements et leurs attitudes ainsi que leurs réponses à des tâches particulières. Avec ces nouvelles connaissances sur les élèves, les FE arrivent à formuler des idées personnelles pour présenter des tâches aux élèves en évitant les confusions et les incompréhensions de leur part. Les données récoltées par Evans (1986) en combinant questionnaires, interviews et observations montrent également que, grâce à leur stage, les 3 FE primaires qu'il a suivis ont mieux pu comprendre l'impact du comportement et de l'attitude de l'enseignant sur les élèves et mieux déceler les actions/réactions des élèves selon que l'enseignant adopte tel ou tel comportement. Les FE rapportent avoir vu suffisamment d'élèves différents pour comprendre que chaque élève a des comportements distincts. Ils disent en avoir appris davantage sur le comportement des élèves et ils ont parfois dû modifier ou changer les conceptions qu'ils avaient des élèves en fonction de ce qu'ils voyaient en classe. Cela leur a également permis d'avoir une vision de la manière dont ils aimeraient se comporter et ne pas se comporter avec leurs propres élèves dans leur future profession. Les FE ont également retenu que leur propre enseignement devait être relié aux niveaux des élèves et que connaître leurs performances était une étape cruciale dans l'élaboration de l'enseignement. À ce propos, le cas de Jack, FE secondaire, est assez représentatif (Borko, Livingston, McCaleb et al., 1988, cités par Borko \& Putnam, 1996). Pendant son premier stage et la première leçon qu'il enseigne, Jack s'est rendu compte qu'il couvrait trop de contenu et il a pris conscience de la réalité : il enseignait à des élèves qui n'avaient jamais appris cette matière auparavant. Cette prise de conscience lui a permis de changer son fonctionnement, de s'adapter aux élèves et à leur rythme.

Deux études portant sur l'effet du premier stage mettent en évidence un retour en arrière dans les croyances des FE lors de l'expérience de terrain. C'est le cas de Hoy et Rees (1977) et de McNeely et Mertz (1990, cités par Kagan, 1992), qui ont observé qu'au début de leur stage les FE qu'ils ont interrogés se sentaient confiants et avaient un sentiment d'auto-efficacité assez élevé. Ils passaient la plupart de leur temps à planifier et à organiser des leçons avec plusieurs activités en portant beaucoup d'attention aux élèves et à la façon dont ils réagissaient aux activités proposées. Alors qu'en fin de stage, ces FE se focalisaient sur le maintien de la discipline et passaient beaucoup moins de temps dans la préparation de leurs leçons. Ils n'organisaient plus qu'une seule activité de nature à ne pas trop encourager des perturbations de classe, ils devenaient moins indulgents avec les élèves et privilégiaient l'ordre et la discipline en se focalisant sur eux-mêmes.

\section{Les stages de façon générale}

Hollingsworth (1989) a mené une étude longitudinale de 5 ans impliquant 14 FE primaires et secondaires suivis à l'aide de questionnaires, d'interviews et d'observations. Elle a observé des évolutions vers une pratique d'enseignement centrée sur la discipline et la façon dont l'enseignant devrait tenir sa classe. La chercheuse a suivi les FE durant les 9 derniers mois de leur formation et a constaté que, lorsqu'ils sont en stage, ils mettent fortement l'accent sur la gestion de leur classe et deviennent excessivement fermes et inflexibles avec les élèves. C'est en discutant avec leur formateur de terrain qu'ils se rendent compte qu'en se focalisant sur la gestion de classe, ils en oublient les besoins des élèves. Perez-Roux (2008) a, quant à elle, observé et questionné Léa, une FE secondaire, de façon intensive pendant deux années de formation et plus spécifiquement pendant ses périodes de stage. Au début, elle se représente l'enseignant idéal comme quelqu'un ayant pour compétences essentielles la connaissance de la psychologie de l'enfant et de l'adolescent, le fait de savoir être en relation avec autrui, de réfléchir sur ses pratiques et d'analyser leurs effets. Alors qu'à la fin de la formation pratique, elle cite une nouvelle fois l'analyse des pratiques et de leurs effets, mais également le 
fait de mobiliser des savoirs pédagogiques et de construire des contenus adaptés à la diversité des élèves. Jones et Vesilind (1996) ont aussi examiné comment les connaissances de $23 \mathrm{FE}$ primaires s'organisaient et évoluaient au moment des stages. Sur la base d'interviews, les chercheuses ont constaté plusieurs changements chez les FE, particulièrement dans leur façon d'adapter et de planifier leurs leçons. Les FE arrivent à s'auto-évaluer et à faire le point sur leurs croyances initiales. Jones et Vesilind (1996) rapportent notamment que, lors des entretiens, ils s'expriment souvent en termes de « je pensais... mais maintenant, je... ». Les FE commencent à lier leurs différentes connaissances apprises lors des cours, ils les intègrent avec plus de sens et les mettent en parallèle avec leur pratique en stage. Ils ont d'ailleurs affirmé que c'est ce qu'ils ont appris pendant les cours théoriques qui leur a permis d'évoluer, alors qu'avant d'entrer dans la phase pratique, ils disent ne pas avoir besoin d'apprendre et que les cours leur sont de peu d'utilité. La coopération avec les maîtres de stage et les expériences que les FE vivent avec les élèves en stage restent les sources dominantes dans leurs changements et leur évolution. Ce sont les élèves qui leur permettent de prendre conscience de leur rôle d'enseignant. En interagissant avec eux en stage, les FE peuvent mieux constater leur efficacité en tant qu'enseignant (Jones \& Vesilind, 1996). Nettle (1998), quant à lui, retire des résultats plutôt mitigés de son étude par questionnaires auprès de 79 FE primaires après leurs premières semaines de stage pratique. Parmi les FE, le chercheur constate tant des évolutions et des changements que des statu quo. Doudin, Pfulg, Martin et alii (2001), utilisant également la technique des questionnaires, interprètent, quant à eux, leurs résultats comme des retours en arrière. Ces chercheurs se sont intéressés aux croyances de 372 FE primaires quant au rôle de l'enseignant dans l'apprentissage, la gestion des difficultés scolaires et l'échec scolaire. En début de formation, les FE expriment en majorité des conceptions socioconstructivistes de l'intelligence et un fort engagement dans la gestion des difficultés et de l'échec scolaire, alors que les FE plus avancés dans leur formation et ayant déjà bénéficié d'une expérience de terrain détiennent des conceptions plus hétérogènes et moins marquées vers un positionnement socioconstructiviste. Pour Doudin, Pfulg, Martin et alii (2001, p. 180), l'expérience de terrain aurait donc un « effet déstabilisateur, voire régressif ». Ng, Nicholas et Williams (2010), qui ont suivi 140 FE secondaires via des questionnaires tout au long d'un cours dans lequel s'inscrivent leurs premiers stages d'observations et de pratiques, concluent aussi à un retour en arrière dans leurs croyances sur le rôle de l'enseignant. Avant le cours, les FE conçoivent l'enseignant comme le maître de la classe, celui qui doit gérer l'ordre et la discipline ; à la fin du cours, ils le voient comme étant avant tout en relation avec ses élèves, mais, après les stages, ils le considèrent à nouveau comme le maître de la classe, celui qui est central et prioritaire dans le processus d'enseignement-apprentissage.

\section{L'entrée en fonction}

Bullough, Knowles et Stokes (1991) ont étudié l'effet de l'entrée en fonction sur trois enseignants secondaires débutants. À travers leur dispositif de recherche alternant des moments d'observation, des interviews et des participations à des séminaires, les chercheurs observent des évolutions chez les trois novices. Leurs conceptions de l'enseignement et de l'apprentissage se peaufinent en devenant plus réalistes et ils acquièrent de nouvelles connaissances sur les élèves qui leur permettent de mieux envisager leur enseignement et la façon de l'adapter à leur classe.

Les observations de Hausoul (2005) témoignent, par contre, d'un retour en arrière dans les croyances des FE. Dans cette étude, on constate effectivement que certains enseignants débutants renouent avec des idées qu'ils ont défendues à un moment de leur formation initiale, plus particulièrement avant un programme de formation 
spécifiquement dévolu à leur faire changer de croyances concernant les effets du redoublement. En effet, deux années après l'étude de Nicolas (2002) détaillée ci-dessus, Hausoul (2005) a pu retrouver 19 de ces 36 FE et les a interrogés à nouveau sur leurs croyances eu égard au redoublement. Tous ces FE avaient eu l'opportunité d'enseigner pendant les deux années écoulées, ce qui permet à Hausoul (2005) d'appréhender l'effet de l'insertion professionnelle sur les croyances d'enseignants débutants. Cette chercheuse constate que tous les enseignants qui étaient " pour " ou " plutôt pour " le redoublement à la fin de leur formation ont été renforcés dans leur conviction et pratiquent le redoublement. Pour la plupart de ceux-ci, la réalité du terrain a conforté leur point de vue. Les 3 enseignants « mitigés » le sont restés. Pour ceux-ci, le fait de faire redoubler un enfant est bénéfique dans certains cas et pas du tout dans d'autres. Enfin, parmi les enseignants qui étaient « plutôt contre " ou " contre " le redoublement, 3 ont changé d'opinion et 3 autres sont restés opposés à cette pratique. Cependant, même ces enseignants convaincus de l'inefficacité de la pratique ont, au cours des deux années écoulées, pris des décisions de redoublement. En définitive, l'étude menée par Hausoul (2005) tend à montrer, sur un petit échantillon, que les effets de la formation initiale ne résistent guère aux pressions sociales du milieu au sein duquel doivent s'insérer les jeunes enseignants, soit qu'ils adaptent leurs croyances à celles qui sont dominantes autour d'eux, soit qu'ils maintiennent leurs convictions personnelles tout en agissant comme l'institution attend qu'ils le fassent, ce qui les place dans une situation inconfortable de dissonance cognitive (Festinger, 1957).

Au regard de toutes ces études, on constate que si les stages sont bels et bien formateurs, les recherches passées en revue ci-dessus montrent qu'ils peuvent néanmoins avoir un effet tant positif que négatif sur les croyances des FE. Les résultats présentés témoignent à la fois d'évolutions et de changements (11 études sur 18) et de retours en arrière ou d'effets à " rebours " (7 études sur 18). Dans un tel contexte, il s'avère difficile de conclure sur l'impact des stages étant donné que l'on ne connaît que peu de chose sur le cadre dans lequel ceux-ci se sont inscrits. Une fois de plus, les différences méthodologiques doivent mener à la prudence. En effet, parmi ces études, les outils quantitatifs et qualitatifs sont tant utilisés séparément qu'ensemble et ce sur des échantillons allant de 1 à 372 FE.

\section{Les effets de la formation initiale au regard des choix méthodologiques}

Dans leur note de synthèse, Crahay, Wanlin, Issaieva et alii (2010) reprochent à Richardson et Placier (2001) de n'avoir pris en compte ni la méthodologie de recherche utilisée ni le type de croyances considérées dans leurs conclusions des études qu'ils recensaient. Cette remarque paraît justifiée. La question du choix d'une méthode qualitative ou quantitative fait, en effet, débat parmi les chercheurs, et certains craignent que les méthodes qualitatives ne constituent une façon d'évaluer pour mieux s'illusionner, pour reprendre le titre d'un article critique d'Huberman (1983).

Le tableau 6 en annexe établit une synthèse des méthodologies utilisées dans les recherches détaillées ci-dessus ainsi que des croyances auxquelles elles se sont intéressées. Il est difficile d'établir un lien strict entre la méthodologie utilisée et la nature des conclusions. On observera toutefois que parmi les 8 études qui ont eu recours à plus de 2 méthodes de mesure, 6 d'entre elles concluent à des effets. 7 sur 12 des études ayant utilisé des questionnaires concluent à des effets positifs. Par contre, en ce qui concerne les autres méthodes de mesure des effets, les ratios sont bas : 2 sur 4 pour les études par interviews, 2 sur 5 pour celles combinant questionnaires et interviews ainsi que pour celles couplant observations 
et questionnaires, et 7 sur 14 pour celles associant observations et interviews. Rappelons que la grande majorité de ces études s'intéresse essentiellement aux croyances sur l'enseignement de façon générale. Seules l'étude de Graham (2005) investigue les croyances sur l'évaluation et celle de Nicolas (2002) les conceptions relatives à la pratique du redoublement. Ce qui différencie le plus ces études, c'est l'échantillon pris en considération : le nombre de FE considérés varie de 1 à 650 . Les recherches administrant des questionnaires portent sur des groupes de FE allant de 36 à 650. Celles qui recourent à des interviews ou à des observations, mais sans questionnaires, investiguent les croyances des FE avec des échantillons allant de 1 à 36. Quant aux études combinant plusieurs techniques dont le questionnaire, leurs échantillons vont de 3 à 228 FE. Dans ce type d'études, c'est évidemment sur un échantillon plus restreint que les outils relevant des méthodes dites qualitatives sont utilisés.

On aurait pu espérer que la combinaison des méthodes de recueil de données tende à s'imposer au sein de recherches sur l'évolution des croyances des enseignants ${ }^{5}$; ce n'est pas le cas comme le montre le tableau 7 en annexe, dans lequel les recherches ont été classées à la fois selon la ou les méthode(s) de recueil de données utilisées et la décennie de réalisation. Notons que ce tableau fait apparaître une diminution du nombre de recherches sur cette thématique. En effet, nous avons pu recenser 11 études menées avant 1991, 21 pendant la décennie 1991-2000 et 13 entre 2001 et aujourd'hui. Ce déclin est regrettable car bien des questions restent non résolues quant au changement de croyances des enseignants.

Bien qu'aucun lien n'apparaisse entre la nature des conclusions et la méthode de mesure des croyances, il y a à ce sujet une question cruciale à traiter. Déjà en 1983, Huberman soulignait que les méthodes appelées qualitatives (interview, observation), par leur prise en compte de la subjectivité des interlocuteurs, de leurs états d'âme, de leurs expressions, de leurs émotions, de leur environnement, visent davantage la qualité de la compréhension que l'exactitude de l'information. Toutefois, le même auteur faisait remarquer que ce serait faire preuve d'une certaine naïveté et d'un manque d'objectivité que de prétendre que les analyses qualitatives des données sont plus valides que les données recueillies et traitées de façon quantitative. Bien plus, Huberman (1983) préconise de procéder si possible à des analyses statistiques rigoureuses sur les données quantitatives. Sur la base de ces réflexions méthodologiques, Verloop, Van Driel et Meijer (2001) rappellent que les connaissances et les croyances des enseignants englobent autant d'éléments personnels que d'éléments partagés par l'ensemble de la communauté enseignante. Ce raisonnement les conduit à rejoindre la position de Huberman (1983) et à préconiser la combinaison des approches : une investigation quantitative à large échelle permettant d'appréhender des éléments partagés et une enquête qualitative permettant d'investiguer les croyances sur un plan plus personnel.

Par ailleurs, on rappellera qu'il ne ressort pas de tendance nette quant aux effets de la formation initiale sur les croyances des FE : seulement 8 études sur 14 tirent des conclusions positives. Les cours théoriques semblent affecter fort peu les FE: 3 études sur 10 concluent à des effets. 11 des 18 études portant sur les stages pratiques et l'expérience de terrain concluent à des effets positifs, mais nous avons constaté que ceux-ci peuvent également faire régresser les FE. Quant aux études portant sur les effets des programmes spécifiquement ciblés sur le travail des croyances initiales des FE, 4 sur 6 sont concluantes. De manière générale, les recherches sont peu informatives sur la mise en œuvre des dispositifs des cours et des stages ainsi que sur l'encadrement dont ont bénéficié les FE ; ceci rend l'interprétation des modifications observées (lorsqu'il y en a) quasi impossible et nous oblige à rester au niveau du simple constat. Notons aussi que les chercheurs 
dans le domaine restent dominés par une vision manichéenne du changement: lorsqu'un changement est établi par les chercheurs, il est synonyme de progrès. Dès lors que les changements ou les modifications dans les croyances des FE ne vont pas dans la direction attendue par les formateurs, les chercheurs parlent de "retour en arrière » ou d'" effet à rebours ». Dans ces études, le changement des croyances des FE au fil de la formation initiale s'apparente donc à une étape dans le développement professionnel tel qu'attendu et voulu par les formateurs. Or, rien n'exclut de penser que le développement professionnel des enseignants puisse emprunter des trajectoires diverses en raison de leurs caractéristiques personnelles et des propriétés du dispositif. Par ailleurs, ces recherches en disent peu sur les raisons des changements observés et sur les difficultés rencontrées pour faire changer les croyances des FE en formation initiale. Certains chercheurs se sont toutefois avancés dans une démarche de type explicative cherchant à comprendre et/ou à spéculer sur les dynamiques de changement et sur les raisons des échecs des tentatives de faire évoluer les croyances des FE. Ce sont ces tentatives que nous examinerons dans la partie suivante de cette note.

\section{CHANGER LES CROYANCES EN FORMATION INITIALE : OUI, MAIS COMMENT ?}

\section{Apprendre à enseigner : un processus complexe}

Apprendre à enseigner est un processus complexe. Durant leur formation, les FE doivent s'approprier de multiples connaissances et habiletés : les contenus et la façon de les transmettre, la façon dont les élèves apprennent, leur motivation et leur façon de s'engager dans les tâches, la gestion d'une classe et d'un groupe d'élèves tous différents par maints aspects, l'évaluation de leurs progrès et bien d'autres aspects qui font partie du quotidien de la profession enseignante (Borko \& Putnam, 1996). Ainsi, tout au long de la formation enseignante, on attend des FE un travail et une réflexion exigeants (Eisenhart, Behm \& Romagnano, 1991) en leur demandant d'acquérir des compétences pédagogiques, de relier la théorie à la pratique, d'utiliser des stratégies pédagogiques innovantes, de résoudre les problèmes qu'ils rencontrent en stage. II leur est demandé simultanément d'élaborer une conception de l'enseignement qui soit à la fois personnelle et compatible avec les prescriptions officielles ainsi qu'avec les savoirs des sciences de l'éducation et de développer une pratique efficace et respectueuse des enfants. Or, au fil de la formation, les FE sont confrontés à des visions de l'enseignement et de l'apprentissage contradictoires (Kagan, 1992 ; Fenstermacher, 1994 ; Tillema \& Knol, 1997). Quant à la réalité de l'enseignement, elle est elle-même complexe (cf. notamment Huberman, 1987 ; Perrenoud, 1983). Or, selon Borko, Eisenhart, Underhill et alii (1991, cités dans Kagan, 1992), les formateurs d'enseignants simplifient excessivement la réalité de l'enseignement lorsqu'ils dispensent les cours théoriques, au point de parfois la travestir. Nombreux sont les FE qui rapportent un manque de liens entre les cours théoriques et leurs expériences de terrain au cours de la formation (Clark \& Peterson, 1986 ; Morine-Dershimer, 1987, 1988 ; Olson, 1981 ; Richardson \& Hamilton, 1994 ; Duffy \& Roehler, 1986 ; Sosniak, Ethington \& Varelas, 1991, cités dans Kagan, 1992). Selon Aitken et Mildon (1991, cités par Kagan, 1992) ainsi que selon Tillema et Knol (1997), le fossé entre les cours théoriques et les expériences de terrain est tellement grand que les FE se retrouvent démunis face aux pratiques pédagogiques à mettre en œuvre en classe et se raccrochent alors à leurs propres conceptions et croyances de l'enseignement. Submergés par les demandes de la formation initiale et ne détenant pas toutes les connaissances adéquates, il n'est pas étonnant de constater que des FE n'arrivent pas à s'engager dans une analyse 
critique de leurs pratiques (Korthagen, 1988). Certains se replient alors sur la culture de l'école dans laquelle ils enseignent et, dans certains cas, finissent par adopter des orientations pédagogiques contraires à celles encouragées par le programme de formation (Eisenhart, Behm \& Romagnano, 1991). Dans ces conditions, il s'avère alors bien difficile d'amorcer le développement professionnel des FE recherché par les formateurs (Korthagen, 1988).

À propos du développement professionnel des enseignants, Huberman (1987) souligne que les modèles classiques qui dessinent des cycles jalonnant la carrière posent deux problèmes. D'abord, les séquences sont prétendues universelles alors que de nombreux facteurs les influencent (facteurs individuels mais aussi environnementaux, politiques, sociaux), Huberman (1987) parle à ce propos « d'uniformisation en masquant des différences intermédiaires ». Ensuite, l'agencement des différentes phases présuppose une continuité. Or, c'est rarement le cas; chaque enseignant peut influencer les étapes qu'il parcourt car chaque enseignant est un individu actif qui réagit aux événements de sa vie professionnelle avec ses caractéristiques propres. Dans une étude portant sur 160 enseignants de tous âges et utilisant des interviews rétrospectives, Huberman (1987) délaisse la recherche de profils généraux pour s'atteler à l'identification de " familles " d'enseignants passant par des étapes semblables. Si les perspectives classiques proposent bien souvent 7 étapes (l'entrée dans la carrière, la stabilisation, l'expérimentation ou la diversification, la remise en question, la sérénité et la distance affective, le conservatisme et la plainte, le désengagement), Huberman (1987) ne retient que les 2 premières car, selon lui, c'est à partir de la phase de stabilisation que les parcours se diversifient. La première étape de l'entrée dans la carrière est caractérisée tant par la découverte, l'enthousiasme et la fierté d'être l'enseignant de sa propre classe que par le choc de la réalité et la perception d'une série de problèmes (voir Veenman, 1984), au centre desquels on trouve le décalage entre les apports théoriques et, surtout, entre les représentations de l'école construites pendant la formation et la réalité du terrain, ce qui débouche sur une attitude plus ou moins prononcée de survie. La deuxième phase, celle de la stabilisation, apparaît généralement entre la $3^{e}$ et la $5^{e}$ année d'enseignement, lorsque l'enseignant s'engage définitivement dans sa profession en ayant le sentiment d'appartenir à l'institution. Selon Huberman (1987), lorsque ces deux phases sont franchies avec succès, les enseignants ont plus de chances de ne pas tomber dans des phases insurmontables de remises en question les menant dans les cas extrêmes au désengagement et, parfois, à la démission. Toujours selon cet auteur, une entrée sur le terrain « facile " et une maîtrise des dimensions-clés de la pédagogie en classe sont des conditions nécessaires pour le développement professionnel futur des FE. Dans son étude, Huberman (1987) constate d'ailleurs que les jeunes enseignants qui entrent sur le terrain de façon difficile et sans véritable enthousiasme sont ceux qui développent le moins bien leurs compétences professionnelles et chez qui la phase d'ennui apparaît le plus tôt. De plus, il rapporte que ces jeunes enseignants se sentent moins bien formés que les "plus anciens enseignants " et voient leur formation comme relevant plus du contrôle que comme une réelle préparation à la profession.

Le manque de liens entre la formation théorique et la réalité du terrain vécue lors des expériences pratiques semble être un premier obstacle au développement professionnel et au changement dans les croyances des FE. Dans les pages qui suivent, nous examinerons comment différents auteurs envisagent la formation théorique d'abord, pratique ensuite, dans la perspective d'induire un changement dans les croyances des FE, mais aussi de promouvoir l'articulation théorie-pratique, un idéal qui fait consensus dans le domaine. 


\section{Comment travailler les croyances pendant les cours " théoriques "?}

Plusieurs chercheurs (Calderhead, 1996 ; Gunstone \& Northfield, 1992 ; Tobin, 1992, cité par Tillema \& Knol, 1997) ont entrepris un examen critique de ce qui est offert aux FE dans les programmes de formation en termes de travail sur leurs conceptions initiales. Ces chercheurs constatent que les programmes ne sont pas du tout reliés aux croyances et aux conceptions que les FE ont de l'enseignement. De plus, la plupart des cours investigués par ces chercheurs ne permettent pas aux FE d'entrer dans des processus de réflexion et d'apprentissage significatifs. Selon Borko et Putnam (1996), les conceptions relatives aux apprenants et à l'apprentissage qui sont valorisées dans les programmes d'enseignement diffèrent, et parfois très nettement, de celles qui sont soutenues par les FE et de celles répandues au sein des écoles dans lesquelles les étudiants effectuent leurs stages. II faut ajouter à ce problème celui pointé par Bramald, Hardman et Leat (1995), qui soulignent la grande diversité entre les cours dans les institutions de formation. Selon eux, il est fréquent que les cours ne soient pas homogènes. Ils observent de grandes différences même à l'intérieur des cours de base qui devraient, en principe, être semblables. Tout ceci génère l'insatisfaction des FE. Ceux interrogés par Chamberlin et Vallance (1991, cités par Kagan, 1992) considèrent que les cours théoriques de leur formation sont trop abstraits pour pouvoir utiliser leurs contenus en classe ; d'autres FE - interrogés par Tillema et Knol (1997) - rapportent que les cours ne sont pas assez focalisés sur les pratiques pédagogiques. À côté de ces articles qui mettent en cause les pratiques de formation les plus courantes, on en trouve d'autres qui proposent des voies pour travailler les croyances pendant les cours théoriques. Nous les examinons dans les sections qui suivent.

\section{Favoriser l'expression des croyances initiales des futurs enseignants}

Selon Feiman-Nemser et Buchmann (1989), les cours théoriques dispensés en formation initiale ne donnent que rarement l'occasion aux FE d'expliciter leurs croyances initiales, ce qui empêche tout travail de réflexion sur celles-ci et, partant, de remise en question. Dans le même esprit, Tillema et Knol (1997) soutiennent que les FE qui suivent des programmes de formation n'abordant pas leurs croyances et ne donnant pas d'occasions d'en parler feront moins d'apprentissages et modifieront moins leurs pratiques pédagogiques ultérieures comparativement aux FE qui auront pu travailler sur leurs conceptions personnelles. Pour Korthagen (1993), il est important que les étudiants puissent prendre conscience de leurs croyances personnelles en les exprimant à travers des verbalisations ou des métaphores et puissent, dans la foulée, les confronter à d'autres conceptions. De même, pour Hollingsworth (1989) ou encore Freeman (1991), permettre aux FE de parler de leurs conceptions implicites est un aspect très important pour leur développement professionnel. Une fois que les conceptions sont explicitées, les FE peuvent les lier à leur propre pratique mais aussi détecter celles qui sont parfois contradictoires ou qui ne fonctionnent pas dans la réalité. Bref, selon tous ces chercheurs, les programmes de formation devraient être organisés de façon à pouvoir expliciter, identifier, comprendre et travailler les croyances initiales des étudiants. En définitive, ces chercheurs recommandent d'inscrire la formation théorique des FE dans le paradigme de la dissonance cognitive de Festinger (1957, cité dans Raths, 2001) : en ayant la possibilité d'exprimer leurs croyances initiales, les FE pourront les confronter à celles partagées par d'autres FE, par leurs formateurs ainsi que par leurs maîtres de stage. C'est en les confrontant avec d'autres qu'ils pourront se rendre compte des convergences et des oppositions. Le point de départ de la formation enseignante devrait donc cibler les perceptions et croyances des FE. Bullough et Stokes (1994), Ball (1988) et Levin et Ammon (1992) ont montré que les programmes dans lesquels les croyances personnelles des FE sont soulevées 
et confrontées à d'autres connaissances peuvent aboutir à un changement chez les FE. Pour Tillema et Knol (1997), dans ce genre de programmes, les nouvelles connaissances doivent être introduites en même temps que les FE exposent leurs croyances, en discutent et les confrontent, ce qui permet des interconnexions.

En définitive, il semble bien que les chercheurs soient unanimes sur la question : si l'objectif est de modifier les croyances des FE, il faut d'abord leur donner l'opportunité de les expliciter (Richardson, 1996 ; Olson, 1993 ; Kagan, 1992 ; Pajares, 1992 ; Fenstermacher, 1979). Kagan (1992) propose à cette fin un dispositif en 3 temps : 1) aider les FE à prendre conscience de leurs croyances implicites et, partant, à les expliciter ; 2) les confronter avec des éléments pouvant déboucher sur une mise en question de leurs croyances en mettant en évidence leurs contradictions internes, leurs incohérences, voire leurs inadéquations par rapport à la réalité de l'enseignement ; 3) donner l'opportunité aux étudiants d'intégrer de nouvelles connaissances, de sorte à différencier les anciennes croyances, voire à remplacer les conceptions totalement inadaptées.

\section{Comment vaincre la résistance de certains futurs enseignants à l'égard d'une démarche auto-réflexive}

Pour pouvoir entreprendre un travail en profondeur sur les croyances initiales, il est nécessaire - argumente McDiarmid (1990) - que les FE soient prêts à revoir leurs connaissances et croyances. S'ils ne sont pas disposés à réfléchir sur leurs conceptions et à les analyser plus finement, ils ne pourront en aucun cas transférer ce qu'ils apprennent de leur propre enseignement. II s'avère donc primordial d'amener les FE à entrer dans cet état d'esprit, de les rendre ouverts à une certaine auto-réflexion car, toujours selon McDiarmid (1990), le domaine des croyances est un domaine qui se rapporte à l'individu tout entier, à son système de valeurs, à ce qu'il est. II n'est donc pas étonnant de constater une résistance chez certains FE à exprimer leurs conceptions et à vouloir les changer. Afin d'éviter de "cabrer " certains FE, Raths (2001) suggère qu'il est peut-être plus acceptable pour eux, mais aussi pour certains formateurs, de présenter la démarche de formation en disant que l'objectif est de renforcer certaines prédispositions dans leurs répertoires de connaissances plutôt que de dire que l'on veut changer leurs croyances. Aborder les croyances dans cette perspective pourrait éventuellement lever certaines résistances et favoriser l'entrée des FE dans le processus de changement. Cette idée, qui nous paraît toutefois éthiquement discutable, n'a pas été - à notre connaissance - objet de recherche. Il faut néanmoins reconnaître que McDiarmid (1990) et, dans la foulée, Raths (2001) pointent un vrai problème qui nous apparaît trop peu investigué par les chercheurs : comment vaincre la résistance de certains FE à l'égard d'une démarche réflexive ? En effet, il n'est pas évident que tous les FE soient disposés à se livrer à cet exercice qui fait rupture avec le métier d'élève auquel ils ont été habitués dans l'enseignement primaire et secondaire. Qui sont ceux qui investissent positivement les démarches auto-réflexives et qui sont ceux qui y résistent? Plus exactement, quelles sont les caractéristiques des premiers et des seconds? Des enquêtes sur cette question seraient utiles, et ceci avant même de mener des recherches visant à aider les seconds à dépasser leurs résistances.

\section{Privilégier l'enseignement de connaissances procédurales}

Vu le caractère professionnel de la formation des enseignants, les connaissances procédurales devraient y être privilégiées. C'est une des idées que suggérait Kagan dès 1992. Selon elle, les FE ont besoin de connaissances procédurales pour acquérir les routines de gestion et celles du processus d'enseignement-apprentissage. Or, toujours selon cette chercheuse, les cours théoriques ne permettent pas d'acquérir les connaissances procédurales nécessaires à la gestion d'une classe, ni les 
connaissances adéquates sur les élèves et sur les contenus à leur enseigner. Plus particulièrement, les cours sur le développement de l'enfant et ceux de psychologie de l'éducation ne fournissent pas aux FE les types de connaissances nécessaires pour leur pratique. La formation nécessiterait une articulation des connaissances déclaratives et des connaissances procédurales (Kagan, 1992).

C'est eu égard à cette hypothèse que l'expérience de Legros et Tochon (1994) prend tout son sens. En effet, ces formateurs-chercheurs ont remanié leur dispositif de formation du cours de didactique de l'oral dans la formation des enseignants primaires de l'université de Sherbrooke selon les besoins exprimés par leurs étudiants. Les FE qu'ils ont interrogés se sont plaints de l'inadéquation entre les cours théoriques et le stage pratique. En conséquence, ces formateurs-chercheurs ont organisé leur cours en « le centrant sur les habiletés procédurales " (Legros \& Tochon, 1994, p. 199) et sur la base de la pédagogie du projet. Les FE étaient placés dans une situation fort semblable à celle de leur stage. Ils devenaient les acteurs principaux du cours et, selon une répartition en groupes, chacun avait pour but de présenter une séquence de cours. De cette expérience, les FE retiennent avoir appris de nombreux acquis et habiletés indispensables à leur future profession. Néanmoins, malgré un syllabus fourni aux FE en tant que complément, ils disent cette fois ne pas avoir bénéficié d'assez de théorie. D'après Legros et Tochon (1994), les FE ont du mal à concevoir un cours dit «théorique " si la théorie n'est concrètement illustrée que par des ressources complémentaires. Pour les FE, le cours idéal « doit reposer sur un dosage équilibré entre la pratique et la théorie pour vraiment correspondre à leurs besoins. Les connaissances acquises en classe doivent être appuyées ou renforcées par des connaissances déclaratives "; connaissances déclaratives qui, selon les FE, doivent être exposées de façon magistrale par le formateur. Cette conclusion de Legros et Tochon (1994, p. 203-204) constitue une nouvelle hypothèse qui mériterait d'être mise à l'épreuve.

\section{Confronter les futurs enseignants à des cas d'enseignement}

Selon McAninch (1993, cité par Raths, 2001) mais aussi Schwab (1978, cité par Raths, 2001) ou encore Cochran-Smith (1991) et Meale (1991, cité par McDermott, Gormley, Rothenberg et al., 1995) si les cours de la formation sont trop détachés et isolés de la réalité que les FE rencontrent en stage, le développement professionnel s'amorcera difficilement. Selon de nombreux chercheurs (notamment Kagan, 1992), en arrivant en stage, les FE ont une vision idéalisée, simpliste et très optimiste du fonctionnement de la classe. II est essentiel de préparer au mieux les FE à entrer sur le terrain mais aussi à travailler sur leurs croyances. L'idéal serait d'intégrer dans chacun de leurs cours de formation une réalité des pratiques de la classe. McAninch (1993, cité par Raths, 2001), sur la base de Schwab (1978, cité par Raths, 2001), propose de confronter les FE à de nombreux cas d'enseignement pendant leur formation théorique. Un travail d'analyse selon différents points de vue (par exemple selon leurs propres croyances, l'approche constructiviste, l'instruction directe, la méthode par projets, etc.) pourrait permettre d'amorcer des changements dans leurs croyances. À notre connaissance, cette hypothèse n'a pas été l'objet d'évaluation empirique.

\section{Travailler à partir de métaphores}

Enfin, pour d'autres chercheurs (par exemple Clark, 1988 ; Grant, 1992 ; Bullough \& Stokes, 1994 ; Calderhead, 1996), le travail avec les métaphores et les images serait le meilleur moyen d'entrer avec les FE dans un travail d'analyse sur leurs croyances initiales. Selon ces chercheurs, et en particulier Calderhead (1996), c'est se méprendre sur la nature des croyances des enseignants que de penser qu'elles sont toutes exprimables sous la forme de propositions formelles. Souvent, elles prennent 
la forme d'images mentales et de scripts. Elles peuvent être «encapsulées » dans leurs expériences de l'école au point qu'elles ne sont pas verbalisables; en tout cas, très difficilement et imparfaitement. Elles se manifestent dans des façons de réagir aux situations ainsi que dans des façons de ressentir celles-ci. Les métaphores offrent dès lors un moyen approprié d'exprimer ces façons de percevoir et/ou de se représenter les situations d'enseignement et d'apprentissage. Pour Bullough et Stokes (1994), les images sont plus que des figures ou des dessins, elles sont de véritables modèles mentaux. Clark (1988) en parle comme des théories implicites. Les images représentent les connaissances cachées et enfouies qui doivent être dévoilées pour être reconstruites (Polanyi, 1958, cité par Bullough \& Stokes, 1994). Pour avoir du sens, elles ont besoin d'être articulées entre elles. D'après Olney (1972, cité par Bullough \& Stokes, 1994), articuler les images, c'est comme se raconter une histoire à soi-même (self-narrative), et ce seraient les métaphores qui traduiraient le thème de l'histoire, tissant le lien entre les images. Les métaphores jouent donc un rôle dans le processus d'exploration et de formation des croyances.

À cet égard, Grant (1992) s'est intéressé à des FE secondaires et observe que, selon la matière à laquelle ils se destinent, les métaphores sont différentes : un FE en sciences parle de magie et d'émerveillement dans les réflexions sur son cours, un FE en histoire voit son sujet comme un jeu dans lequel les étudiants viennent explorer des évènements historiques et un FE en anglais considère son cours comme un voyage à travers lequel les étudiants doivent sans cesse imaginer et raconter des choses. Les images que les FE se construisent vont les guider dans leur façon d'aborder leur formation et elles peuvent avoir d'importantes répercussions sur la manière dont ils vont développer leur pratique enseignante (Calderhead \& Robson, 1991). Selon Wubbels (1992, cité par Calderhead, 1996, p. 719), voir l'enseignant comme un "dompteur de lions, comme un capitaine de navire ou comme un maître de cérémonie " peut avoir des effets totalement différents sur la façon de réagir du FE dans les différentes situations de classe et sur le comportement que, probablement, il prévoit de la part des élèves.

Pour devenir enseignant, il faut d'abord se former une identité d'enseignant. Utiliser les métaphores pour explorer cette identité, c'est aborder les images de soi en tant qu'enseignant, mais aussi celles des élèves et de leur rôle. Selon Calderhead (1996), les FE débutent leur formation en ayant en tête non pas des théories formelles mais des images de ce qu'est l'enseignement. Incrustées dans ces images se trouvent leurs croyances sur le rôle du bon enseignant et celui des élèves, sur la façon dont on enseigne et dont on apprend, sur la vie en classe (Kagan, 1992). Si les FE ne possèdent pas une image d'eux-mêmes en tant qu'enseignant, ils peuvent - affirme Kagan (1992) - se retrouver totalement perdus lors de leurs premières interventions en classe. Il s'avère alors nécessaire de les aider à prendre conscience de cette image pour qu'ils puissent se projeter au mieux dans leur futur métier. À cet égard, les métaphores se révéleraient être une base idéale à utiliser dans les cours de la formation. En sus, toujours selon Kagan (1992), la présence réelle d'un modèle, à savoir un enseignant chevronné, un maître de stage qui peut montrer au FE comment il se remet en question, réfléchit et prend du recul sur ses croyances pédagogiques, est ce qui facilite le mieux la projection dans le métier.

Peu de recherches visant à faire évoluer les croyances des FE se sont attachées à travailler les métaphores; dans la recension discutée dans la première partie, nous n'avons recensé que 2 études : celle de Bullough et Stokes (1994) sur 22 FE secondaires (destinés à des disciplines différentes) bénéficiant d'une séquence de travail intensif basé sur l'utilisation de métaphores rapporte des résultats positifs engrangés sous la forme d'évolution personnelle (cf. tableau 4) et l'étude quasi expérimentale de Leavy, McSorley et Boté (2007) sur 124 FE primaires bénéficiant 
soit de 10 semaines de terrain, soit uniquement de cours théoriques, qui travaillait les métaphores des FE et les comparait avant et après le traitement (terrain ou théorie) sans résultats positifs. En définitive, il semble bien que les hypothèses formulées principalement par Calderhead (1996) et Kagan (1992) concernant l'intérêt des métaphores n'ont pas été suffisamment prises en considération par les chercheurs puisque peu d'entre eux les ont mis à l'épreuve du terrain.

\section{Les stages et l'entrée sur le terrain, des opportunités de développement professionnel ? À quelles conditions ?}

Pour Bullough et Stokes (1994), c'est en stage que les FE sont vraiment confrontés à la réalité de la classe, aux conceptions des enseignants en fonction et à celles des autres personnes (directeurs, parents, etc.) de l'établissement qui les accueille. C'est dans ce contexte que les FE doivent être encouragés à mobiliser leurs croyances relatives à l'enseignement et, le cas échéant, à les faire évoluer ou à en adopter d'autres (Bullough \& Stokes, 1994). De même, pour Evans (1986), l'expérience de terrain est le moment idéal pour confronter les images et les métaphores des FE au réel, leur faire découvrir de nouvelles représentations et faire évoluer leurs croyances initiales. Or, d'après Kagan (1992), la durée des stages n'est pas assez longue en formation initiale pour acquérir une vision réaliste de l'enseignement et des contextes scolaires. De plus, l'expérience de terrain ne se focalise pas assez sur les besoins essentiels au bon développement professionnel. Plus précisément, pour cette chercheuse, les stages ne donnent pas toujours la possibilité au FE de: 1) confirmer et valider son image en tant qu'enseignant, ni d'utiliser les connaissances sur les élèves pour la modifier ; 2) automatiser les procédures managériales et instructionnelles requises pour enseigner ; 3) être confronté à une dissonance cognitive et se poser la question de la pertinence de ses images et croyances personnelles. Au lieu de s'adresser à chacun de ces besoins, l'organisation des stages semble se faire de façon idiosyncratique selon le type de relation qui se développe entre le stagiaire et son maître de stage (Kagan, 1992). Dans un tel contexte, il importe de se questionner dans un premier temps sur ce qui est offert aux FE lors de leurs expériences pratiques en formation initiale et d'envisager dans un second temps comment établir un travail sur leurs croyances pendant ces moments de terrain. C'est l'intérêt des points ci-dessous.

\section{Activités offertes aux futurs enseignants au cours de leurs expériences pratiques}

Plusieurs chercheurs ont déterminé les activités offertes aux FE lors de leurs expériences pratiques afin de les aider dans leur développement professionnel et dans l'évolution de leurs conceptions. Olson et Bruner (1974, cités par Evans, 1986) distinguent deux modalités d'expérience de terrain :

- l'observation d'un enseignant en train d'enseigner ; le FE est supposé apprendre en regardant son maître de stage agir et construire par imitation ou apprentissage vicariant (Bandura, 1986) un répertoire de pratiques ;

- l'enseignement en classe ; le FE apprend en agissant directement et en ajustant ces pratiques en fonction des conséquences de ses actions ; dans ce cas, l'apprentissage se ferait selon un processus du type de celui conceptualisé par Skinner (1968) en termes d'apprentissage opérant.

Ryan (1982, cité par Evans, 1986) décompose ces 2 modalités en 5 types d'activité : 1) l'observation, 2) la participation en classe (premiers contacts avec les élèves, passage dans les rangs, aide), 3) le tutorat, 4) l'enseignement en petits groupes et 5) l'enseignement en grand groupe (voir tableau 8 en annexe). 
Selon Ryan (1982, cité par Evans, 1986), il n'y a pas de consensus sur la durée, l'ordre et même la combinaison de ces activités pendant l'expérience de terrain. Toujours selon Ryan (1982, cité par Evans, 1986) mais aussi Wilson (1975, cité par Evans, 1986), ce serait l'observation qui aurait le plus d'effets sur les FE car elle leur permettrait de considérer le contexte de la classe dans sa globalité et de prendre conscience de tout ce qui s'y passe. S'appuyant sur les hypothèses de ces auteurs, Evans (1986) a analysé la façon dont les FE observaient l'enseignement lors de leur première entrée en stage. II constate que les 4 premiers jours sont déterminants car c'est à ce moment-là que les FE s'imprègnent de la dynamique de la classe et façonnent leurs conceptions. De ses observations sur les FE, Evans (1986) dégage la séquence suivante : le 1er jour, les FE regardent le style de l'enseignant, sa méthode d'enseignement; le $2^{e}$ jour, ils regardent les règles établies, les routines mises en place ; le $3^{e}$ jour, ils s'intéressent à la façon dont l'enseignant gère la discipline, son autorité et, le $4^{\mathrm{e}}$ jour, ils s'imaginent à la place de l'enseignant en essayant d'anticiper leur rôle d'enseignant. Les FE récoltent donc un maximum d'informations les premiers jours d'observation car elles sont nécessaires pour débuter au mieux leur premier enseignement. D'après Evans (1986), pour qu'une expérience de terrain soit efficace, il faut passer à la fois par l'observation de la classe et par celle de l'enseignement. D'une part, le FE ne doit pas seulement reproduire ce que fait son maître de stage, il doit comprendre pourquoi il agit de cette façon et quelles alternatives sont possibles ; d'autre part, le FE ne doit pas agir de suite car il doit d'abord avoir compris un minimum des stratégies d'enseignement et d'apprentissage pour pouvoir s'adapter au mieux à la classe. Dans la formation à l'enseignement, il serait donc important de préparer les FE à l'observation et à l'enseignement en classe. Toujours selon Evans (1986), les formateurs doivent aider les FE à pointer les éléments à observer, à prendre en compte, à comprendre et à intégrer lorsqu'ils seront dans la classe ; selon la conceptualisation d'Ausubel (1968), les formateurs doivent fournir des advance organizers aux FE avant de les plonger dans le terrain. Ils doivent aussi les aider à anticiper les réactions des élèves et à planifier leur enseignement en les mettant en situation avec des exemples concrets. Pour que les FE apprennent de leur expérience de terrain, il est nécessaire que des moments de discussion et de régulation soient mis en place avec le maître de stage ou le formateur avant le stage, mais aussi pendant et après pour réguler au mieux les actions des FE (Evans, 1986).

Sur la base de ses recherches, Hollingsworth (1989) suggère quant à elle qu'il pourrait y avoir un ordre séquentiel dans la prise de contact avec le terrain. L'objectif de ce séquençage est d'éviter les phénomènes de surcharge cognitive dont on connaît les effets néfastes sur l'apprentissage (Crahay, 1999) et ainsi de favoriser la prise d'informations pertinentes. Dans cette perspective, les étudiants ne devraient plus se concentrer sur tous les aspects de l'enseignement à la fois et pourraient se focaliser petit à petit sur les différents aspects qu'ils rencontrent lors de leurs expériences sur le terrain : la gestion de classe, l'apprentissage et l'enseignement du contenu, l'évaluation des élèves, leurs difficultés d'apprentissage... Ici, comme chez Evans (1986), on retrouve l'idée que la prise de contact avec le terrain doit être préparée et qu'il devrait être opportun de guider la prise d'information des FE en leur fournissant des advance organizers (Ausubel, 1968).

\section{Devenir enseignant, un développement identitaire}

Le métier d'enseignant ne se ramène pas à un répertoire de pratiques et de croyances ; il y a une posture, une identité à construire. Nombreux sont les auteurs qui postulent que l'identité professionnelle des enseignants se développe essentiellement dans des situations professionnelles en interaction avec des êtres humains (Beckers, 2007). Les expériences de terrain apparaissent donc comme 
étant les moments les plus formateurs de cette identité : se retrouver dans une école, avec une classe à prendre en charge, plonge directement le FE dans la pratique professionnelle et l'amène à se socialiser en tant qu'enseignant. Pour Nitonde et Paquay (2011), les effets positifs des stages sont pluriels ; ils permettent aux FE : 1) de développer des compétences professionnelles et de construire leur identité professionnelle ; 2) d'acquérir une image réaliste du métier ; 3) de se socialiser à la profession. Cet enthousiasme n'est pas partagé par tous les auteurs. Ainsi, pour Evans (1986), même si les stages renvoient une image de la réalité du terrain, l'expérience pratique y reste artificielle car, en cours de formation, les FE réalisent leurs stages dans un but certificatif. Ainsi, ils observent la classe et leur maître de stage en récoltant les informations dont ils ont besoin pour leurs leçons, pour répondre aux attentes de leurs formateurs et/ou à celles du maître de stage (si celui-ci participe à l'évaluation du FE). Quand ils observent leur maître de stage enseigner, ils s'intéressent d'ailleurs plus au « comment faites-vous pour... » plutôt qu'au « pourquoi faites-vous cela... » (Evans, 1986). Toujours selon Evans (1986), ils se focalisent directement sur ce dont ils auront besoin pour réussir leur stage. Ceci s'expliquerait par le fait que les FE conservent une identité étudiante forte en début de formation (Beckers, 2007). Cela est plutôt logique étant donné leur statut de FE et leur manque de pratique sur le terrain. Sous leur identité d'étudiant, les FE restent donc fort attachés aux critères d'évaluation lors de leurs stages en ne se projetant pas encore dans leur profession future : ce qui compte pour eux c'est la réussite de leur stage, et leur vie professionnelle leur paraît encore lointaine. Evans (1986) a pointé cette identité forte d'étudiant chez les FE qu'il a observés en deuxième année de formation, avant, pendant et après leur premier stage. D'après ses résultats, les FE considèrent l'expérience de terrain comme une chance de se préparer aux plus longues semaines de pratique qui les attendent l'année suivante et comme une opportunité de montrer leur capacité à être enseignant. De façon complémentaire, les FE expriment aussi une grande anxiété face à l'évaluation de leurs professeurs et de leur maître de stage qui influence leurs notes de fin d'année (Evans, 1986).

Tout en partageant l'idée qu'en entrant en stage les FE sont imprégnés d'une identité étudiante forte qui domine leurs réflexions, Dubar (2000) soutient que, petit à petit, celle-ci va évoluer vers une identité d'enseignant. Pour cet auteur, au fil de ses interactions avec la classe, le FE capte l'image que les élèves mais aussi les autres acteurs scolaires lui renvoient. Dubar (2000) parle à cet égard de l'identité pour autrui, celle qui permet de se construire une image de soi dans le métier. C'est en prenant conscience de cette image renvoyée principalement par les élèves et en la confrontant avec sa propre image de lui en tant qu'enseignant que le FE développe sa projection dans le métier. Dans ce processus, les formateurs et les maîtres de stage ont également un rôle à jouer : ils doivent aider le FE à se construire une identité professionnelle d'enseignant.

\section{La rencontre avec les élèves, un facteur de changement}

Comme on vient de le lire, nombreux sont les auteurs qui soulignent combien la rencontre des élèves affecte le développement professionnel des FE. Lorsqu'ils arrivent en stage, les FE semblent en savoir vraiment peu sur les élèves et sur leur façon d'apprendre (Borko \& Putnam, 1996 ; Kagan, 1992). Or, la connaissance des élèves et des processus d'apprentissage est considérée de façon unanime comme un facteur-clé du métier d'enseignant. Dans tous les programmes de formation, des cours théoriques y sont consacrés. Cependant, pour Evans (1986) mais aussi Kagan (1992) et Borko et Putnam (1996), les stages offriraient les meilleures opportunités de développer cette catégorie de connaissances, car les FE ont alors l'occasion d'interagir avec les élèves et de les observer dans le contexte de la classe. Au 
fil de leurs interactions avec les élèves, les FE peuvent mieux les comprendre et donc prendre du recul sur leurs conceptions initiales en pointant là où elles sont incorrectes ou inappropriées (Kagan, 1992). Les maîtres de stage et les formateurs doivent être attentifs à l'acquisition de ces connaissances chez les FE pour pouvoir revenir avec eux sur l'évolution de leurs croyances à propos des élèves.

La gestion de la classe est un autre aspect important à travailler lors du stage avec les FE. Ceci est d'ailleurs une préoccupation majeure des FE lorsqu'ils arrivent en stage pour prendre en main une classe. Divers auteurs - notamment, Borko et Putnam (1996), Hollingsworth (1989) et Kagan (1992) - soulignent l'importance de cette préoccupation (concern dans la littérature anglo-saxonne) chez les FE, et ceci constitue une différence par rapport aux connaissances sur les élèves, qui sont un souci second chez les FE. Selon les auteurs cités ci-dessus, il appartient aux maîtres de stage et aux formateurs de pousser les FE à s'intéresser au processus d'enseignement et d'apprentissage dès qu'ils semblent à l'aise avec la gestion de classe. C'est en travaillant les interactions avec les élèves, l'observation de leurs comportements face à l'apprentissage et à l'enseignement dispensé par le FE dans un contexte de classe qu'il aura établi, qu'il pourra prendre conscience des effets de ses actions pédagogiques et les ré-adapter si nécessaire. Le FE sera alors plus facilement amené à revoir ses conceptions initiales. C'est du moins l'hypothèse formulée par les trois auteurs mentionnés.

Le maître de stage, maillon fort du développement professionnel des futurs enseignants

Selon Jacknicke et Samiroden (1991, cités par Kagan, 1992), la relation entre le stagiaire et le maître de stage est déterminante dans l'évolution professionnelle du FE. Cette relation joue un rôle majeur dans la façon dont les FE assimilent les connaissances du métier (Chamberlin \& Vallance, 1991, cités par Kagan, 1992). À ce propos, des FE interrogés par Legros et Tochon (1994) rapportent que le manque d'affinité et de confiance avec le maître de stage a eu une influence négative sur la façon dont ils ont vécu leur stage. Le maître de stage doit faire en sorte que le FE se considère comme un apprenant libre d'essayer et de faire des erreurs pour se rendre compte du besoin de changer certaines de ses croyances personnelles (Hollingsworth, 1989). C'est seulement lorsque le maître de stage donne la possibilité au FE de mettre en œuvre ses propres idées que celui-ci peut réaliser que certaines d'entre elles doivent être modifiées. Selon Guskey (1986), il est important de donner aux FE des occasions d'exercer de nouvelles pratiques pour qu'ils puissent eux-mêmes constater leurs succès ou leurs échecs. En partant de la pratique que les FE mettent en place en classe, les maîtres de stage servent alors de guide pour permettre aux FE de tester les méthodes vues lors des cours et les contenus enseignés. C'est de cette façon qu'ils pourront alors ajuster voire remplacer leurs conceptions initiales. Le maître de stage doit donc laisser le FE faire ses propres leçons et choisir ses propres objectifs pour les élèves. En imitant aveuglément le maître de stage, il n'y aura pas d'acquisition durable des procédures de la classe (Bullough, 1991).

Ces considérations s'inscrivent dans le courant conceptuel du self-regulated learning. L'idée centrale est que l'auto-évaluation par les FE de leur fonctionnement constitue un processus crucial de leur développement professionnel. Cette idée est exprimée avec force par Kagan (1992), selon qui les FE doivent comprendre ce qui fonctionne et ce qui ne fonctionne pas en classe ainsi que le pourquoi de ces (dys) fonctionnements. Ils doivent faire preuve d'auto-réflexion sur leurs comportements, leurs croyances et leurs images d'eux-mêmes en tant qu'enseignant. Afin de favoriser le développement de cette démarche d'auto-régulation, une collaboration entre les 
FE, les maîtres de stage et les formateurs doit être mise en place aussi bien avant que pendant et après la prise en charge de la classe par les FE.

Dans le même esprit, McDiarmid (1990) argumente pour que la collaboration entre le maître de stage et le FE soit favorisée dès le début de l'expérience de terrain afin de permettre au FE de s'inscrire au mieux dans son rôle d'enseignant. À partir de là, pense McDiarmid (1990), le FE pourra mieux travailler sur ses propres conceptions de l'enseignement et ses mises en œuvre en classe et entrer dans un processus de changement de pratiques et de croyances. Toujours selon cet auteur, il est essentiel que les FE soient préparés à entrer sur le terrain, tout comme les maîtres de stage doivent être prêts à les accueillir (McDiarmid, 1990). De même, pour Hollingsworth (1989), les maîtres de stage doivent fournir aux FE des opportunités d'appliquer les idées importantes présentées lors des cours théoriques, notamment les cours de méthode ; pour cela, ils doivent être guidés par les formateurs. Car, toujours selon Hollingsworth (1989), si les FE ne sont pas amenés à appliquer les concepts vus aux cours, ils ne pourront pas les comprendre en profondeur. II faut donc les aider à transférer les concepts appris aux cours en classe de stage. Ces idées sont reprises par Kagan (1992), qui soutient que la collaboration des FE avec des maîtres de stage qui facilitent l'intégration de nouvelles connaissances en travaillant sur leurs croyances initiales constituerait la meilleure expérience possible pour le développement professionnel des FE. Selon elle, une collaboration de cette qualité requiert que les maîtres de stage qui accueillent les FE s'inscrivent eux-mêmes dans une approche développementale et réflexive.

Il importe, dans le cadre de cette note de synthèse, de souligner que ces idées, aussi séduisantes soient-elles, n'ont pas été testées dans des études empiriques - du moins, pas à notre connaissance.

\section{Des moments d'échanges pour intégrer apports de l'expérience et réflexion théorique}

Pour tous les auteurs que nous avons consultés, il va de soi que l'expérience de terrain par les stages ne peut se ramener à une immersion dans la réalité. $\mathrm{Si}$ certains soulignent l'importance de la préparation des FE avant qu'ils n'entament les stages, si d'autres (parfois, les mêmes) mettent en avant l'importance de la collaboration avec le maître de stage, d'autres encore proposent de mettre en place des séances de travail pendant ou après les stages pour réfléchir l'expérience de terrain. Ainsi, pour Nitonde et Paquay (2011), l'idéal serait d'aménager des moments d'échanges et de réflexion sur les actions mises en place lors du stage tout en liant la théorie apprise lors des cours. Selon ces chercheurs, c'est en parlant de ses représentations, de ses pratiques et de ses savoirs qu'un FE pourra établir des liens et des oppositions entre eux et ainsi entrer dans un processus de transformation. Dans le même esprit, Balslev, Tominska et Vanhulle (2011) insistent sur l'importance des séminaires d'intégration accompagnant les stages, au sein desquels les FE sont invités à discuter de leur expérience pratique en la reliant à leur formation théorique. Van Nieuwenhoven et Labeeu (2010) proposent quant à eux des entretiens dits " à chaud " qui sont réalisés entre le FE et son maître de stage ou son formateur directement après un moment en stage pour favoriser la réflexion sur ce qui a été mis en place et son efficacité. De Cock, Wibault et Paquay (2006), eux, parlent d'entretiens dits "réflexifs " qui portent sur une pratique professionnelle que le FE a mise en place, le but étant de l'analyser, de constater ses effets, de l'ajuster. Le FE doit pouvoir verbaliser les représentations qu'il avait de son action et la transformation qu'il peut en faire. Pour ces auteurs, faire du tutorat et des régulations avec les FE sur ce qu'ils observent et mettent en œuvre en stage leur fait prendre conscience de l'importance du processus d'enseignement-apprentissage au sens 
large. Balslev, Tominska et Vanhulle (2011) nous informent cependant des limites de ce genre d'entretiens. Les chercheuses constatent de leurs études que bien souvent les maîtres de stage ou les tuteurs proposent des solutions toutes faites aux FE, en ne leur laissant que peu de place pour une réflexion professionnelle. Pour éviter cela, il importe selon Beckers (2007) d'entrer dans le système interprétatif du FE, en le guidant dans sa réflexion personnelle et en le laissant s'exprimer et penser lui-même ses actions.

Poursuivant le même objectif, l'appropriation des savoirs professionnels par les FE, Vanhulle (2009) a développé une autre approche consistant à encourager les enseignants en formation à tenir une sorte de carnet de bord qui les suit tout au long de leur formation et à y rédiger des textes réflexifs portant tout aussi bien sur les cours théoriques reçus que sur les expériences de terrain. Analysant les productions des FE, cette chercheuse met en évidence 1) « une dynamique adaptative " selon laquelle le FE se conforme aux attentes et aux prescrits de la formation et 2) « des démarches de compréhension, de reformulation, de délibération critique en termes de pertinence pratique " (Vanhulle, 2009, p. 21). Du point de vue adopté dans cette note de synthèse, il est important de souligner l'importance quantitative et qualitative des données empiriques recueillies par cette chercheuse qui, ce faisant, montre l'intérêt du dispositif mis en place par elle-même en collaboration avec une formatrice de Haute école pédagogique.

\section{DISCUSSION ET CONCLUSIONS}

La revue de la littérature à laquelle nous avons procédé débouche sur des constats complémentaires. Dans la première partie, les études recensées concernant l'effet des dispositifs de formation sur l'évolution des croyances des FE fournissent des résultats plus que mitigés. Dans de nombreux cas, les chercheurs se résignent à conclure des effets de la formation que les croyances n'ont pas bougé au cours de celle-ci, que ce soit de l'effet du programme en général, des cours théoriques ou des stages et de l'expérience de terrain. À nos yeux, au terme de cette recension de recherches, il convient de questionner l'adéquation des dispositifs actuels de formation des enseignants, dans la mesure où il est attendu de ceux-ci qu'ils fassent évoluer les conceptions des FE. Quant aux recherches passées en revue dans la seconde partie, elles fournissent des éléments explicatifs de l'inefficacité des dispositifs actuels de formation des enseignants et suggèrent des pistes d'amélioration qu'il importe encore, pour la plupart, d'explorer.

\section{De l'inadéquation des dispositifs actuels de formation des enseignants à changer leurs croyances}

Les recherches sur l'évolution ou les changements de croyances des FE en cours de formation initiale ont été menées selon des schémas méthodologiques très diversifiés. Cette hétérogénéité méthodologique rend difficile la synthèse des recherches et de leurs résultats. Ceux-ci sont également très hétérogènes. À côté des recherches qui ne concluent à aucune modification, d'autres mettent en évidence des évolutions ou des changements de croyances chez les FE. Cependant, par delà la variabilité des résultats, il faut reconnaître qu'il existe peu de données attestant un changement en profondeur et à long terme dans les croyances initiales des FE au terme de la formation. Dans la seconde partie, nous nous sommes notamment intéressés à la façon dont les croyances sont abordées lors des cours 
et des stages. II ressort des recherches ayant exploré cette question d'une part un manque d'articulation entre la formation théorique et la formation pratique et, d'autre part, peu de moments de travail ciblés spécifiquement sur les croyances des FE. Ceci expliquerait d'une certaine manière pourquoi les changements de croyances en formation initiale ne s'opèrent qu'en surface et à court terme et n'aboutissent pas à de réels changements conceptuels (Kagan, 1992 ; Borko \& Putnam, 1996 ; Richardson \& Placier, 2001).

Au terme de cette note de synthèse, dans laquelle les différents composants de la formation ont été explorés (cours théoriques, stages,...), plusieurs raisons peuvent être avancées pour expliquer la stabilité des croyances des FE lors de la formation initiale et, partant, l'inefficacité de celle-ci à les faire évoluer :

- nous retiendrons tout d'abord le fossé entre les cours théoriques dispensés en formation et la réalité du terrain rencontrée en stage ou lors de l'entrée en fonction. Cette absence de lien entre la théorie et la pratique est relatée de façon quasi unanime au sein des recherches (par exemple Clark \& Peterson, 1986 ; Morine-Dershimer, 1987, 1988 ; Olson, 1981 ; Richardson \& Hamilton, 1994 ; Kagan, 1992 ; Legros \& Tochon, 1994 ; Tillema \& Knol, 1997) ;

- deuxièmement, le travail sur les conceptions initiales des FE est peu présent dans la formation. Nombreux sont les chercheurs qui soulignent l'importance de susciter soit des dissonances cognitives dans la réflexion des $\mathrm{FE}$, soit des confrontations de conceptions entre les FE entre eux, entre les FE et les formateurs, entre les FE et leur maître de stage (par exemple Feiman-Nemser, 1983 ; Hollingsworth, 1989 ; Olson, 1993 ; Richardson, 1996). Or, des dispositifs sollicitant les confrontations de points de vue ou suscitant des dissonances cognitives touchent inéluctablement aux systèmes de valeurs de l'être humain, à son histoire personnelle et à ses expériences passées ainsi qu'à son image de soi et, plus précisément, à sa perception de sa capacité à se transformer. De ce fait, leur mise en place s'avère délicate. II n'est pas évident de faire émerger des conceptions enfouies au plus profond de la cognition de jeunes adultes et de s'engager dans un processus complexe d'analyse de celles-ci. À leur entrée en formation initiale, les FE sont peu préparés à un travail réflexif sur leurs propres croyances, ceci afin de les mettre en question pour les faire évoluer et les modifier;

- s'ajoute aux deux déficiences susmentionnées le fait que, lorsque les FE entrent pour la première fois sur le terrain, ils ne possèdent pas assez de connaissances adéquates des élèves et de la façon de gérer la classe. À cet égard, il a été mis en évidence que la formation développait davantage l'acquisition de connaissances déclaratives au détriment de connaissances procédurales et d'habiletés (par exemple Kagan, 1992 ; Borko \& Putnam, 1996 ; Tillema \& Knol, 1997).

Avec de telles carences, il n'est pas étonnant de constater que les FE se retrouvent démunis quand ils sont confrontés au contexte scolaire. D'autant plus qu'ils doivent à la fois acquérir de nombreuses compétences, renvoyant aux diverses facettes que recouvre le métier d'enseignant, tout en devant répondre à un grand nombre d'exigences certificatives au sein de leur formation. Il leur est alors plus facile de se replier sur leurs propres conceptions ou sur celles qu'ils rencontrent sur le terrain (Eisenhart, Behm \& Romagnano, 1991). Par ailleurs, il convient encore de noter que bon nombre d'enseignants méconnaissent les résultats des recherches en éducation (Gore \& Gitlin, 2004 ; Joram, 2007). Leurs croyances s'élaborent donc le plus souvent indépendamment de ce savoir. Dans un tel contexte, et étant donné que les conceptions initiales sont fortement enracinées dans le système de pensées, la difficulté à faire évoluer et changer les croyances des FE n'est pas 
étonnante (Richardson \& Placier, 2001). Cependant, cette difficulté ne doit pas renvoyer à une impossibilité de faire changer les croyances en formation initiale. La revue de littérature présentée dans la seconde partie de cette note de synthèse permet d'imaginer des pistes de travail à explorer.

\section{Des pistes à explorer pour favoriser le développement professionnel des futurs enseignants}

Dès lors que l'on se fixe le but de répondre aux carences énoncées plus haut et de focaliser davantage la formation sur le changement des croyances initiales, il paraît opportun de synthétiser ici les apports des nombreuses recherches (par exemple Kagan, 1992 ; Ball, 1992 ; Bullough \& Stokes, 1994 ; Legros \& Tochon, 1994 ; Borko \& Putnam, 1996) qui ont investigué la façon de moduler la formation initiale pour qu'elle puisse davantage articuler la théorie et la pratique afin de favoriser au mieux l'évolution des croyances et, partant, le développement professionnel des FE. Nous déclinerons cette discussion finale selon les deux composants principaux de la formation initiale : les cours théoriques d'abord, les stages ensuite.

Des cours théoriques pour amorcer l'évolution des croyances des futurs enseignants

Notre recension des recherches a été articulée autour de quatre idées programmatiques ; nous les rappelons ci-dessous, dans l'ordre dans lequel nous les discutons:

- vaincre la résistance de certains FE à l'égard d'une démarche auto-réflexive ;

- favoriser l'expression des croyances initiales des FE ;

- confronter les FE à des cas d'enseignement ;

- privilégier l'enseignement de connaissances procédurales.

II semble bien qu'il y ait un consensus parmi les chercheurs dans le domaine sur l'idée qu'il faut concevoir la formation des enseignants de manière à développer et/ou renforcer leurs capacités et leur propension à l'auto-réflexion et à l'autorégulation (cf. notamment Raths, 2001 ; Paquay, 2004 ; Beckers, 2007). Cette finalité, aussi légitime soit-elle, est exigeante et, partant, difficile à réaliser. Elle sous-entend que l'enseignant doit se remettre en question de façon quasi permanente. II ne s'agit donc pas seulement d'acquérir des connaissances déclaratives et procédurales, ni même d'apprendre une façon de faire la classe, ce qui est probablement une représentation initiale du métier chez les FE. La formation des enseignants se conçoit désormais comme un travail sur soi à l'instar d'une psychothérapie ; une idée qui est exprimée par les expressions souvent interchangeables de développement personnel et développement professionnel. Ceci est clair notamment pour McDiarmid (1990) qui suggère que le point de départ de la formation pourrait consister à encourager les FE au changement personnel. Dans le même esprit, des auteurs comme Lortie (1975) ou Kagan (1992) indiquent avec force que les croyances psychopédagogiques se rapportent à l'individu tout entier, à son système de valeurs et, par conséquent, qu'il peut être délicat de le remettre en question. II faut donc obtenir des FE un engagement fort dans un processus qui vise à les transformer en tant qu'individus. En définitive, l'enseignant doit devenir une sorte d'idéal humain sur le plan professionnel. II doit non seulement agir juste, mais penser juste. Sa pratique doit être adaptée aux caractéristiques des élèves tout en visant à les amener à un dépassement d'eux-mêmes à des fins d'apprentissage et de développement cognitif, affectif et social. Cette exigence déjà élevée ne suffit pas. Le bon enseignant doit en plus surveiller en permanence les effets de sa pratique, les évaluer, en juger la pertinence et le cas échéant la transformer tout en 
se montrant capable de la justifier en invoquant des connaissances scientifiques validées et idéalement récentes et, in fine, s'avérer apte à faire la théorie de sa pratique. Le lecteur l'aura deviné : de notre point de vue, obtenir l'engagement des FE dans pareil processus de développement personnel/professionnel n'est pas une mince affaire. Or, cette problématique a, en définitive, été fort peu explorée. Seuls Evans (1986), McDiarmid (1990) et Raths (2001) en font un point-clé. La plupart des autres chercheurs font comme s'il allait de soi que les FE adhèrent à cette vision de leur formation comme un processus au cours duquel ils ont pour mission de se transformer en profondeur et, notamment, de réviser leurs croyances en matière d'enseignement, d'apprentissage, d'évaluation, de redoublement, etc. Comme nous l'avons déjà mentionné, des recherches devraient être menées sur cette question afin d'identifier précisément quelles sont les représentations des FE quant à ce qui est attendu dans le processus formatif. Une fois ces représentations recensées et les résistances identifiées, il conviendra d'étudier comment favoriser l'engagement des FE dans cette démarche visant le développement personnel et dans quelle mesure cela est possible avec tous. II s'agirait de s'interroger sur le meilleur moment d'amorcer cette auto-réflexion. On peut, en effet, se demander si, dans un premier temps, il ne convient pas de fournir aux FE les moyens cognitifs et comportementaux de faire la classe. On a vu ci-dessus que la préoccupation première des FE a trait à leur capacité à gérer le groupe des élèves. II paraît opportun de s'interroger sur les étapes que devrait respecter un programme de formation visant à faire évoluer les croyances des FE. Notons encore que cette conception de la formation des enseignants ciblée sur l'auto-régulation soulève des questions éthiques trop peu discutées. Notamment, que faire avec les FE qui refusent explicitement ou implicitement d'entrer dans cette d'auto-transformation ? Que faire lorsque des FE limitent leur projet de formation à l'apprentissage des « bonnes » pratiques ?

Que l'on invite les FE à expliciter leurs croyances au début des cours théoriques et que ceux-ci soient conçus de sorte à susciter des dissonances cognitives et/ ou des confrontations de points de vue entre différentes personnes ne soulève, de notre point de vue, aucune question éthique. Notons qu'il s'agit apparemment d'une piste de formation déjà en partie validée. Le seul résultat positif engrangé dans la première partie concerne les programmes de formation spécifiquement conçus pour transformer les pratiques. 4 des 6 études recensées montrent des changements dans les croyances des FE (Bullough \& Stokes, 1994 ; Ball, 1988 ; Levin \& Ammon, 1992 ; Nicolas, 2002). Dans les quatre cas, les programmes de formation misaient à la fois sur l'explicitation des croyances initiales et sur des procédés visant à bousculer celles-ci ; procédés s'inscrivant dans le paradigme de la dissonance cognitive (Festinger, 1957) ou dans celui de la confrontation de points de vue (Doise \& Mugny, 1981 ; Mugny, 1985).

Très peu d'études ont, en définitive, exploré la piste du travail avec les métaphores. Or, plusieurs chercheurs (Evans, 1986 ; Bullough \& Stokes, 1994 ; Raths, 2001) font I'hypothèse que cette façon de procéder serait la plus pertinente pour atteindre les croyances des enseignants. II importe également de noter que cette suggestion est à mettre en relation avec une question théorique non résolue, celle concernant le format des croyances : celles-ci sont-elles, toutes ou partiellement, verbalisables selon un format propositionnel ou, au contraire, sont-elles, toutes ou seulement certaines, stockées en mémoire sous un mode iconique ? Les recherches sur la façon de changer les croyances des FE pourraient enrichir ce débat théorique.

Le manque d'articulation entre la théorie et la pratique est soulevé de façon quasi unanime par les FE (par exemple Kagan, 1992 ; Legros \& Tochon, 1994). Pour y remédier, la préparation du premier contact avec le terrain et des stages en général paraît cruciale. Les cours théoriques peuvent y contribuer à condition 
d'être conçus dans cette perspective. Une façon de faire est suggérée par Evans (1986) et Nitonde et Paquay (2011). Selon ces auteurs, les formateurs pourraient proposer des cas concrets d'enseignement, que les FE analyseraient sur la base des théories psychologiques et éducationnelles enseignées mais également à partir de leurs propres conceptions. McAninch (1993, cité dans Raths, 2001) envisage même de proposer des points de vue théoriques différents selon les différents courants pédagogiques afin de permettre une réflexion plus profonde et davantage de liens entre la théorie, les croyances et les pratiques. Une autre voie, pas nécessairement opposée, consiste à mettre l'accent sur le besoin de connaissances procédurales exprimé par les FE (par exemple Borko \& Putnam, 1986 ; Kagan, 1992). À cet égard, la recherche de Legros et Tochon (1994) est particulièrement intéressante car elle révèle qu'en définitive c'est un juste équilibre entre connaissances déclaratives et procédurales que réclament les FE. II faut regretter que cette étude soit unique en son genre.

\section{La rencontre avec le terrain : comment en tirer le meilleur parti ?}

L'initiation à la pratique de la classe peut se faire selon diverses modalités déclinées par Olson et Bruner (1974, cités dans Evans, 1986) et complétées par Ryan (1982, cité dans Evans, 1986). Ces modalités sont résumées dans le tableau 8 en annexe.

Dans la première partie, nous avons constaté que les évaluations quant aux effets des stages ne permettent pas de conclure de façon nette à ce sujet. Les résultats présentés dans le tableau 5 révèlent des évolutions et des changements (11 études sur 18), mais aussi des retours en arrière ou des effets à " rebours " (7 études sur 18). Afin de préciser l'analyse, nous avons distingué les effets de l'expérience pratique (entendue comme une expérience d'enseignement hors du contexte de formation) de ceux du premier stage et de ceux des stages en général. Ceci nous a amenés à constater plus de résultats positifs pour les premiers stages (6 études sur 8) que pour les stages en général (3 études sur 6) ou pour l'expérience pratique (1 étude sur 2). II importe cependant de souligner qu'il n'a pas été possible de décrire plus précisément quelles activités spécifiques avaient été proposées aux FE. En définitive, ces études ne disent rien sur la bonne façon d'organiser l'expérience du terrain. Si l'on en croit Ryan (1982, cité dans Evans, 1986) mais aussi Wilson (1975, cité dans Evans, 1986) et Evans lui-même, l'observation pendant les stages s'avère être un moment crucial pour les FE car c'est à ce moment-là qu'ils récoltent toutes les informations essentielles à l'organisation des leçons qu'ils dispenseront par la suite à la classe (Evans, 1986). Toujours selon cet auteur, il est opportun de commencer par l'observation de la classe et puis de faire l'expérience directe de l'enseignement. Et Evans (1986) ajoute encore une autre condition de qualité : la préparation des FE aux éléments sur lesquels être attentifs lors de la phase d'observation, ceci afin que les FE ne se focalisent pas sur certains éléments peu importants au détriment d'autres plus importants. Toujours selon cet auteur, une bonne préparation aux stages diminuerait l'anxiété des FE au moment de la prise de contact avec le terrain. Ces idées sont séduisantes. À notre connaissance, leurs effets n'ont pas été évalués empiriquement. II reste donc du travail à faire.

Cette considération peut s'étendre aux autres suggestions ou recommandations faites par différents chercheurs. Ainsi, Iorsque Nitonde et Paquay (2011) ou Van Nieuwenhoven et Labeeu (2010) préconisent des rencontres entre le formateur et le maître de stage, suggérant que celles-ci permettraient de pouvoir aborder en stage des contenus et des méthodes plus proches de ceux qui sont enseignés en formation, on est tenté de conclure d'emblée à la justesse de la recommandation. II n'en reste pas moins qu'on n'en a pas la preuve empirique. Et l'on peut encore dire de même eu égard aux idées de McDiarmid (1990) et de Kagan (1992) qui plaident 
en faveur d'une rencontre entre le maître de stage et le stagiaire dès le début du stage, supposant que celle-ci favoriserait une relation coopérative de confiance et serait l'occasion de confronter une première fois les croyances de chacun sur le déroulement du stage. Et il en va de même de l'idée de Guskey (1986), pour qui il est important que le maître de stage donne la possibilité aux FE d'exercer de nouvelles pratiques pour qu'ils puissent constater par eux-mêmes leurs succès et leurs échecs. Bref, il semble évident que la qualité de la relation entre le FE et son maître de stage est cruciale dans le processus de développement professionnel des enseignants, mais combien d'études empiriques portent spécifiquement sur ce sujet ? Que sait-on de véritablement établi à ce sujet par delà les convictions des uns et des autres?

Outre les idées mentionnées ci-dessus, d'autres encore mériteraient des explorations empiriques quant à leurs effets réels. Ainsi, Borko et Putnam (1996) préconisent d'utiliser, en cours de stage, les connaissances sur les élèves comme facteur de dissonance cognitive. Selon eux, c'est à partir des comportements et des interactions des élèves que le FE pourra le mieux constater son (in)efficacité mais également prendre conscience de son image d'enseignant auprès des élèves. À partir de là, il pourra, toujours selon ces auteurs, travailler sur ses propres conceptions et modifier celles qui semblent erronées ou celles qui ne lui conviennent pas. L'idée est à nouveau séduisante, d'autant plus qu'elle repose sur la théorie de la dissonance cognitive, qui semble être un bon moyen de susciter un travail réflexif de la part des FE sur leurs croyances. Mais qui a vraiment mis cette idée en pratique, selon quelles modalités précises et avec quels effets ? Et il est encore possible de développer une argumentation similaire à propos de l'importance du retour de stage et des séminaires d'intégration que certains recommandent d'organiser à ce moment-là. Quelles modalités d'organisation de ces séminaires d'intégration engendrent vraiment les effets attendus ?

À partir de l'inventaire des différentes suggestions recensées dans la section « Les stages et l'entrée sur le terrain, des opportunités de développement professionnel ? À quelles conditions ? » de la deuxième partie de notre note, il est possible d'esquisser une grille qui synthétise les caractéristiques de préparation et d'accompagnement des stages (voir tableau 9 en annexe).

Croyances, connaissances et valeurs, plus une pincée de sentiment d'efficacité...

Comme expliqué tout au début de cette note de synthèse, le changement dans les croyances des FE peut être conceptualisé en termes de substitution (avant le FE pensait $A$ et maintenant il pense B) ou d'évolution (les croyances $A$ des FE progressent vers $B$ ). Dans un cas comme dans l'autre, les chercheurs considèrent que les croyances $B$ sont préférables aux croyances $A$. Or, la question se pose de savoir ce qui légitime cette position. En vertu de quel(s) principe(s) les chercheurs peuvent-ils parler d'évolution et plus largement considérer que certaines croyances sont meilleures que d'autres?

Prenons le cas de l'étude de Leavy, McSorley et Boté (2007). Ces chercheurs considèrent qu'il n'y a pas eu d'évolution de croyances de la part des FE qui sont allés en stage et concluent à un renforcement de leurs croyances initiales de type behavioriste. Mais peut-être les FE ont-ils raison de conserver leurs croyances initiales de type behavioriste en ce qui concerne la gestion de la classe. Et cette interrogation peut être adressée à Hoy et Rees (1997) qui concluent à un retour en arrière, constatant que les FE sont passés d'une conception de l'enseignant et de l'ordre au centre du processus vers une conception de l'élève et de son apprentissage au centre du processus d'enseignant avant de reprendre leur première conception. 
En vertu de quel(s) principe(s), de quelle(s) valeur(s) ou de quelle(s) connaissance(s) scientifique(s) peut-on parler de retour en arrière ? Rappelons en effet que Kirschner, Sweller et Clark (2006) contestent la supériorité des pratiques socioconstructivistes, considérées aujourd'hui par beaucoup comme les plus souhaitables, par rapport à des pratiques plus structurantes. En réalité, il faut reconnaître que, dans de nombreux cas, nous ne savons pas quelles sont les meilleures croyances à avoir (Raths, 2001). Dans beaucoup de cas, il peut y avoir controverse. Ainsi, en ce qui concerne la gestion de la classe et de l'enseignement, pour Leavy, McSorley et Boté (2007), c'est dans la direction du socioconstructivisme qu'il faut faire évoluer les conceptions des FE, alors que, pour Kirschner, Sweller et Clark (2006), ce serait vers des conceptions inspirées du behaviorisme et de la psychologie cognitive ${ }^{6}$.

Le problème posé ici est difficile. Comment envisager une formation sans définir un but, des objectifs à atteindre ? Si toutes les croyances et toutes les pratiques se valent, il n'y a pas de raisons qui légitiment le fait d'inciter les FE à changer de croyances. On peut considérer à l'instar de Paquay (1994) que l'enseignant est acteur social et qu'en tant que tel il lui faut " adhérer aux objectifs de l'enseignement et il les met en œuvre en jouant son rôle dans l'institution » (p. 10). Ainsi, actuellement, vu que la plupart des systèmes d'enseignement ont opté pour l'approche par compétences, l'enseignant doit adopter les pratiques en conformité avec cette approche. Sans réflexion critique ? Si c'est cela la perspective, cela circonscrit l'auto-réflexion et l'auto-régulation dans les limites des prescrits institutionnels. Cela signifie qu'un Freinet ou un Paulo Freire seraient condamnés au silence.

Pour Raths (2001), l'enseignant est un professionnel et c'est sur la base des caractéristiques du professionnel que l'on peut éventuellement envisager de dresser les conceptions à enseigner aux FE. Ces caractéristiques sont reprises dans le tableau 10 en annexe. Assurément, la liste est intéressante. On remarquera cependant que l'ensemble définit une posture ou une démarche plutôt que des croyances vers lesquelles faire évoluer les FE. Or, si l'on s'accorde avec Belensky, Clinchy, Goldberger et al. (1986, cités par Raths, 2001) qui soutiennent que le développement professionnel des FE dépend bel et bien de l'évolution de leurs croyances, il est nécessaire de se donner des critères pour définir de façon légitime ce qu'est une évolution de croyances à l'opposé d'un retour en arrière.

Lorsque, comme c'est le cas à propos du redoublement, la recherche apporte des preuves solides en faveur ou contre une pratique précise, le critère s'impose : la croyance la meilleure est la plus rationnelle, c'est-à-dire celle qui est soutenue par le plus de preuves scientifiques. Cependant, il faut reconnaître que, dans de nombreux cas, la littérature de recherche ne permet pas de trancher entre différentes alternatives. Prenons le cas de l'enseignement de la lecture. À ce sujet, malgré d'importantes avancées en psychologie de la lecture (cf. pour des synthèses Martinet \& Rieben d'une part, et Bianco d'autre part, dans Crahay \& Dutrévis, 2010), certaines polémiques persistent. Peut-être faut-il considérer, dans la lignée de la conception de l'enseignant comme étant un professionnel, qu'il y a évolution lorsque le FE peut justifier ses croyances en invoquant des connaissances psychologiques, sociologiques, didactiques ou autres, ou encore qu'il peut fonder ses croyances sur des principes axiologiques légitimes ? Ainsi, on considérerait qu'il y a progrès lorsque le FE peut expliquer pourquoi il défend telle ou telle croyance à condition que l'argument soit considéré comme valide, c'est-à-dire appuyé sur des connaissances au moins partiellement validées ou sur des valeurs légitimes (par exemple l'égalité de réussite à l'opposé de l'élitisme). À ce critère de la justification, on pourrait ajouter celui de la cohérence : il y aurait progrès lorsque le FE défend un ensemble de croyances de plus en plus cohérentes et articulées entre elles. Un dernier critère qui pourrait être retenu serait un progrès en matière de sentiment d'efficacité. De 
nombreux auteurs (par exemple Ashton, 1984 ; Hoy \& Woolfolk, 1990 ; Woolfolk Hoy, Davis \& Pape, 2006) considèrent que le sentiment d'auto-efficacité a une forte influence sur le développement professionnel et le changement des conceptions personnelles. Notons que cette façon de conceptualiser l'évolution des croyances a des conséquences sur les moyens pour les mesurer. II semble en effet difficile dans une telle perspective de recourir uniquement à l'utilisation de questionnaires.

En conclusion, le changement des croyances en formation initiale ne peut être considéré comme un fait impossible ; les limites méthodologiques et conceptuelles des recherches recensées dans la première partie ne permettent pas une telle affirmation. De plus, les études passées en revue dans la seconde partie ouvrent des pistes nombreuses qui restent à explorer. Une question d'ordre épistémologique doit impérativement, selon nous, être prise à bras le corps par la communauté de ceux qui travaillent dans le domaine de la formation des enseignants : celle de la définition de critères permettant de définir ce qu'est une évolution ou un changement positif de croyances.

Fanny Boraita

Fanny.Boraita@unige.ch Université de Genève, DAISS (Développement, apprentissage et intervention en situations scolaires)

Marcel Crahay

Marcel.Crahay@unige.ch Université de Genève, DAISS et université de Liège, laboratoire Approches psychopédagogiques et apprentissages scolaires

\section{NOTES}

1 Recherche soutenue par le Fonds national suisse de la recherche scientifique (FNS).

2 Ainsi, comme le notent Crahay, Wanlin, Issaieva et alii (2010), les auteurs ne se sont pas encore mis d'accord quant à la façon de distinguer les termes tels que croyances, conceptions, théories implicites, connaissances, représentations, etc. Comme l'ont fait remarquer ces auteurs, les Anglo-saxons, las de se quereller quant à la distinction des croyances et des connaissances, ont fini par adopter l'expression "Teachers beliefs and knowledge ". Quant à l'expression "théorie personnelle ", elle est le cœur de recherches qui s'intéressent à l'articulation des croyances des enseignants et, plus précisément, à la question de savoir si ceux-ci coordonnent leurs croyances et leurs connaissances de façon cohérente.

3 Notons, sur le plan méthodologique, que seuls Tillema et Knol (1997) et Nicolas (2002) recourent à un plan quasi expérimental comparant soit deux types de programme, soit un groupe expérimental et un groupe de contrôle. Les autres études sont menées selon le schéma avant/après qui ne permet pas de contraster les changements observés à la suite de l'intervention avec ce qui se passe lorsque celle-ci n'est pas appliquée.

4 Ce sont les études qui comparent les FE qui ont déjà une expérience du terrain (enseignement, prise en charge d'une classe) avec ceux qui n'ont bénéficié que de cours théoriques et qui n'ont donc aucune expérience du terrain.

5 Désormais, de plus en plus de chercheurs soulignent les mérites et les limites tant des approches quantitatives que des approches qualitatives et plaident pour leur complémentarité (Crahay, 2006 ; Paquay, 2006 ; Vanderberghe, 2006).

6 Car le monde de la formation des FE reste marqué et même clivé par l'opposition behaviorisme versus constructivisme. Cette opposition nous apparaît quant à nous obsolète, mais elle subsiste dans les textes et dans les recherches et véhicule une position normative des chercheurs qui n'est, par ailleurs, que rarement explicitée et justifiée. La persistance de cette opposition traduit - pensons-nous - la nécessité d'adopter une position quelque peu normative dès lors que l'on forme des $\mathrm{FE}$, car, dans une certaine mesure au moins, former implique de recommander.

\section{BIBLIOGRAPHIE}

ASHTON P. (1984). " Teacher efficacy: A motivational paradigm for effective teacher education ». Journal of Teacher Education, vol. 35, n 5, p. 28-32.

AUSUBEL D. P. (1968). Educational Psychology: A Cognitive View. New York : Holt, Rinehart and Winston. 
BALL D. L. (1988). « Unlearning to teach mathematics ». For the Learning of Mathematics, vol. 8 , no 1 , p. 40-48.

BALSLEV K., TOMINSKA E. \& VANHULLE S. (2011). « Le jour où je serai dans ma classe ce sera différent. Entretiens de stage et construction de savoirs professionnels ». Les Sciences de l'éducation - Pour l'ère nouvelle, vol. 44, n² 2, p. 85-102.

BANDURA A. (1986). Social foundations of thought and action: A social cognitive theory. Englewood Cliffs (États-Unis) : Prentice-Hall.

BECKERS J. (2007). Compétences et identité professionnelle. Bruxelles : De Boeck.

BOLIN F. (1990). « Helping student teachers think about teaching: Another look at Lou ». Journal of Teacher Education, vol. 41, no 1, p. 10-19.

BOOK C., BYERS J. \& FREEMAN D. (1983). «Student Expectations and Teacher Education Traditions with Which We Can and Cannot Live ». Journal of Teacher Education, n० 9.

BORKO H. \& PUTNAM R. T. (1996). «Learning to teach ». In D. L. Berliner \& R. C. Calfee, Handbook of educational psychology. New York: Macmillan, p. 673-708.

BRAMALD R., HARDMAN F. \& LEAT D. (1995). "Initial teacher trainees and their views of teaching and learning ». Teaching and Teacher Education, vol. 11, n० 1, p. 23-31.

BULLOUGH R. V. (1991). " Exploring personal teaching metaphors in preservice teacher education ". Journal of Teacher Education, vol. 42, n० 1, p. 43-51.

BULLOUGH R. V., KNOWLES J. G. \& CROW N. A. (1991). Emerging as a teacher. London : Routledge.

BULLOUGH R.V. \& STOKES D. (1994). " Analyzing personal teaching metaphors in preservice teacher education as a means for encouraging professional development ». American Educational Research Journal, vol. 31, no 1, p. 197-224.

CABAROGLU N. \& ROBERTS J. (2000). « Development in student teachers' pre-existing beliefs during a 1-year PGCE programme ». System, vol. 28, p. 387-402.

CALDERHEAD J. (1988). "The contribution of field experiences to student primary teachers' professional learning ». Research in Education, n० 40, p. 34-49.

CALDERHEAD J. (1996). «Teachers: Beliefs and knowledge ». In D.-L. Berliner \& R.-C. Calfee, Handbook of educational psychology. New York: Macmillan, p. 673-708.

CALDERHEAD J. \& ROBSON M. (1991). "Images of teaching: Student teachers' early conceptions of classroom practice ». Teaching \& Teacher Education, n० 7, p. 1-8.

CHENG M., CHAN K., TANG S. \& CHENG A. (2009). "Pre-service teacher education student's epistemological beliefs and their conceptions of teaching ». Teaching and Teacher Education, vol. 25, p. 319-327.

CHIN R. \& BENNE K. (1969). "General strategies for effecting changes in human systems ». In W. Bennis, K. Benne \& R. Chin, The planning of change ( $2^{\mathrm{e}} \mathrm{e} d$.). New York : Holt, Rinehart and Winston, p. 32-59.

CIVIL M. (1993). "Prospective elementary teachers' thinking about teaching mathematics ». Journal of Mathematical Behavior, vol. 12, no 1, p. 79-109.

CLARK C. M. (1988). «Asking the right questions about teacher preparation: Contributions of research on teacher thinking ". Educational Researcher, vol. 17, n², p. 5-12.

CLARK C. M. \& PETERSON P. L. (1986). «Teachers' thought processes ». In M. C. Wittrock, Handbook of research on teaching ( $3^{e}$ éd.). New York: Macmillan, p. 255-296.

COCHRAN-SMITH M. (1991). «Learning to teach against the grain ». Harvard Educational Review, vol. 61, n०3, p. 279-310.

CRAHAY M. (1999). Psychologie de l'éducation. Paris : PUF.

CRAHAY M. (2004). «Peut-on conclure à propos des effets du redoublement ? ». Revue française de pédagogie, no 148, p. 11-23.

CRAHAY M. (2006). "La recherche qualitative en éducation : dégager le sens et démêler la complexité ». In L. Paquay, M. Crahay \& J.-M. De Ketele (dir.), L'analyse qualitative en éducation. Des pratiques de recherche aux critères de qualité. Bruxelles : De Boeck, p. 33-53.

CRAHAY M. \& DUTRÉVIS M. (2010). Psychologie des apprentissages scolaires. Bruxelles : De Boeck.

CRAHAY M., ORY P. \& BORAITA F. (2012). « Les croyances des enseignants concernant le redoublement évoluent-elles au cours de leur formation initiale?". Annales scientifiques de la faculté de psychologie et sciences de l'éducation de l'Université " Ovidius » de Constanta, p. 31-51. 
CRAHAY M., WANLIN P., ISSAIEVA É. \& LADURON I. (2010). "Fonctions, structuration et évolution des croyances (et connaissances) des enseignants ". Revue française de pédagogie, no 172, p. 85-129.

CUBAN L. (1988). "Constancy and change in schools (1880s to the present) ». In P. W Jackson, Contributing to educational change: Perspectives on research and practice. Berkeley (États-Unis) : McCutchan, p. 85-106.

DE COCK G., WIBAULT B. \& PAQUAY L. (2006). « Le processus de réflexion sur la pratique professionnelle : des modèles à construire ". In J. Desjardins, H. Hensler, O. Dezutter \& A. Beauchesne (dir.), Développer des compétences en enseignement : quelle place pour la réflexion professionnelle ? Montréal : ACFAS.

DOISE W. \& MUGNY G. (1981). Le développement social de l'intelligence. Paris : Interéditions.

DOUDIN P.-A., PFULG L., MARTIN D. \& MOREAU J. (2001). " Entre renoncement et engagement. Un défi pour la formation continue des enseignants ». In L. Lafortune, C. Deaudelin, P.-A. Doudin \& D. Martin. La formation continue. De la réflexion à l'action. Québec: Presses de I'Université du Québec.

DUBAR C. (2000). La crise des identités : l'interprétation d'une mutation. Paris : PUF.

DUFFY G. \& ROEHLER L. (1986). "Constraints on teacher change ». Journal of Teacher Education, vol. 35, p. 55-58.

EISENHART M., BEHM L. \& ROMAGNANO L. (1991). "Learning to teach: Developing expertise or rite of passage? ». Journal of Education for Teaching, vol. 17, p. 51-71.

EVANS H. (1986). " How do early field experiences influence the student teacher? ". Journal of Education for Teaching, vol. 12, no 1, p. 35-46.

FEIMAN-NEMSER S. (1983). "Learning to teach ». In L. Shulman \& G. Sykes, Handbook of teaching and policy. New York : Longman, p. 150-171.

FEIMAN-NEMSER S. \& BUCHMANN M. (1989). "Describing teacher education: A framework and illustrative findings from a longitudinal study of six students ». The Elementary School Journal, vol. 89, n³, p. 365-377.

FEIMAN-NEMSER S., MCDIARMID G. W, MELNICK S. L. \& PARKER M. (1989). Changing beginning teachers' conceptions: A description of an introductory teacher education course (Research Report 89-1). East Lansing (Etats-Unis) : National Center for Research on Teacher Education, College of Education, Michigan State University.

FENSTERMACHER G. D. (1979). "A philosophical consideration of recent research on teacher effectiveness ». Review of research in education, vol. 6, p. 157-185.

FENSTERMACHER G. D. (1986). "Philosophy of research on teaching: Three aspects ". In M.-C. Wittrock, Handbook of research on teaching (3e éd.). New York : Macmillan, p. 37-49.

FENSTERMACHER G. D. (1994). "The knower and the known: the nature of knowledge in research on teaching ". Review of research in education, vol. 20, p. 1-54.

FESTINGER L. (1957). A theory of cognitive dissonance. Stanford (États-Unis) : Stanford University Press.

FLORIO-RUANE S. \& LENSMIRE T. (1990). “ Transforming future teachers' ideas about writing instruction ». Journal of Curriculum Studies, vol. 20, n० 3, p. 277-289.

FREEMAN D. J. (1991). "To make the tacite explicite: teacher education, emerging discourse, and conceptions of teaching ". Teaching and Teacher Education, vol. 9, no 5-6, p. 439-454.

GITLIN A., BARLOW L., BURBANK M., KAUCHAK D. \& STEVENS T. (1999). "Pre-service teachers' thinking on research: implications for inquiry oriented teacher education ». Teaching and Teacher Education, vol. 15, p. 753-769.

GORE J. \& GITLIN A. (2004). « [Re]Visioning the academic teacher divide: Power and knowledge in the educational community ". Teachers and Teaching: Theory and Practice, vol. 10, p. 35-58.

GRAHAM P. (2005). "Classroom-based assessment: Changing knowledge and practice through preservice teacher education ». Teaching and Teacher Education, vol. 21, p. 607621.

GRANT G. E. (1992). "The sources of structural metaphors in teacher knowledge: Three cases ". Teaching and teacher Education, vol. 8, n० 5-6, p. 433-440.

GUNSTONE R. \& NORTHFIELD J. (1992). «Conceptual Change in Teacher Education: The Centrality of Metacognition ". Communication au colloque de l'American Educational Research Association. 
GUSKEY T. R. (1986). "Staff development and the process of teacher change ». Educational Researcher, vol. 15, no 5, p. 5-12.

HAUSOUL E. (2005). Quels sont les effets de l'insertion professionnelle des enseignants sur leurs représentations sociales et, plus précisément, sur leur représentation de l'échec scolaire? Mémoire de licence, sciences de l'éducation, université de Liège (Belgique).

HOLLINGSWORTH S. (1989). "Prior beliefs and cognitive change in learning to teach ». American Educational Research Journal, vol. 26, n² 2, p. 160-189.

HOLT-REYNOLDS D. (1992). «Personal history-based beliefs as relevant prior knowledge in coursework ». American Educational Research Journal, n²9, p. 325-349.

HOY W. K \& REES R. (1977). "The bureaucratic socialization teachers ». Journal of Teacher Education, vol. 28, no 1, p. 23-36.

HOY W. K. \& WOOLFOLK A. E. (1990). "Socialization of student teachers ». American Educational Research Journal, n²7, p. 279-300.

HUBERMAN M. (1983). "S'évaluer pour s'illusionner? Promesses et écueils de l'évaluation adaptative/interactive des innovations scolaires ". Cahier du Groupe des chercheurs romands en pédagogie, no 9, p. 1-38.

HUBERMAN M. (1987). "Le cycle de vie professionnelle des enseignants secondaires ". Cahiers de la section des sciences de l'éducation de l'université de Genève, no 54.

JONES M. G. \& VESILIND E. M. (1996). "Putting practice into theory: Changes in the organization of preservice teachers' pedagogical knowledge ». American Educational Research Journal, n०33, p. 91-117.

JOHNSTON S. (1992). "Images: The way of understanding the practical knowledge of student teachers ". Teaching and Teacher Education, vol. 8, p. 123-136.

JORAM E. (2007). “Clashing epistemologies: Aspiring teachers', practicing teachers', and professors' beliefs about knowledge and research in education ". Teaching and Teacher Education, vol. 23, p. 123-135.

KAGAN D.M. (1992). "Professional growth among preservice and beginning teachers ". Review of educational research, no 62, p. 129-169.

KIRSCHNER P.-A., SWELLER J. \& CLARK R.-E. (2006). «Why Minimal Guidance During Instruction Does Not Work: An Analyis of the Failure of Constructivist, Discovery, Problem-based, Experiential, and Inquiry-Based Teaching ». Educational Psychologist, vol. 41, n०2, p. 75-86.

KORTHAGEN F. A. J. (1988). "The influence of learning orientations on the development of reflective teaching ». In J. Calderhead, Teachers' professional learning. Philadelphie (États-Unis) : Falmer Press, p. 35-50.

KORTHAGEN F. A. J. (1993). «Two modes of reflection ». Teaching and Teacher Education, vol. 9, no 3, p. 317-326.

LANIER J.-E. \& LITTLE J.-W. (1986). « Research on teacher education ». In M.-C. Wittrock, Handbook of research on teaching ( $3^{e}$ éd.), p. 527-569.

LEAVY A. M., McSORLEY F. A. \& BOTÉ L. A. (2007). «An Examination of What Metaphor Construction Reveals about the Evolution of Preservice Teachers' Beliefs about Teaching and Learning ". Teaching and Teacher Education, vol. 23, n० 7, p. 1217-1233.

LEGROS C. \& TOCHON F. V. (1994). «Une tentative d'intégration théorie/pratique au sein de la formation générale professionnelle des enseignants : une pièce en trois actes ». Spirale, no 12, p.175-196.

LEVIN B. B. \& AMMON P. A. (1992). "The development of the beginning teachers' pedagogical thinking: A longitudinal analysis of four case studies ». Teacher Education Quarterly, vol. 19, no 4, p. 19-37.

LORTIE D. C. (1975). Schoolteacher. Chicago : University of Chicago Press.

MARKEL S. (1995). Acquiring pratical knowledge: A study of development through observations of student teaching practice and dialogues of community. Tucson : University of Arizona. En ligne : http://arizona.openrepository.com/arizona/handle/10150/187313 (consulté le 9 juin 2013).

MATTHEOUDAKIS M. (2007). "Tracking changes in pre-service EFL teacher beliefs in Greece: A longitudinal study ». Teaching and Teacher Education, vol. 23, p. 1272-1288.

McDERMOTT P., GORMLEY K., ROTHENBERG J. \& HAMMER J. (1995). "The influence of classroom practica experiences on student teachers' thoughts about teaching ». Journal of Teacher Education, vol. 46, n 3, p. 184-191. 
McDIARMID G. W. (1990). «Challenging prospective teachers' beliefs in an early field experience: A quixotic undertaking? ". Journal of Teacher Education, vol. 29, no 1, p. 2129.

McDIARMID G. W. (1992). "What to do about differences? A study of multicultural education for teacher trainees in the Los Angeles Unified School District ". Journal of Teacher Education, vol. 43, n० 2, p. 83-93.

MORINE-DERSHIMER G. (1987). "Practical examples of the practical argument: A case in point ». Educational Theory, vol. 37, p. 395-407.

MORINE-DERSHIMER G. (1988). "Premises in the practical arguments of preservice teachers ». Teaching and Teacher Education, vol. 4, p. 215-229.

MUGNY G. (1985). Psychologie sociale du développement cognitif. Berne : Lang.

NETTLE E. B. (1998). "Stability and change in the beliefs of student teachers during practice teaching ». Teaching and Teacher Education, vol. 14, n० 2, p. 193-204.

NG W., NICHOLAS H. \& WILLIAMS A. (2010). "School experience influences on preservice teachers' evolving beliefs about effective teaching ". Teaching and Teacher Education, vol. 26, p. 278-289.

NICOLAS A. (2002). Peut-on lutter contre la foi qu'ont les futurs enseignants dans les effets positifs du doublement? Mémoire de licence, sciences de l'éducation, université de Liège (Belgique).

NITONDE F. \& PAQUAY L. (2011). « Vers quelles pratiques de stage en formation initiale des enseignants du secondaire ? Analyse des conceptions des enseignants de l'ENS au Burundi ». Éducation et formation, n०295, p. 143-164.

OLSON J. (1981). "Teacher influence in the classroom ». Instructional Science, no 10, p. $259-275$.

OLSON M. R. (1993). "Knowing what counts in teacher education ». Communication à la Canadian Association of Teacher Educators, Canadian Society of Studies in Education (Ottawa, Canada).

PAJARES M.-F. (1992). « Teachers' Beliefs and Educational Research: Cleaning up a Messy Construct ». Review of Educational Research, vol. 62, n० 3, p. 307-332.

PAQUAY L. (1994). "Vers un référentiel des compétences professionnelles de l'enseignant ? ». Recherche et formation, no 16, p. 7-38.

PAQUAY L. (2004). L'évaluation des enseignants : tensions et enjeux. Paris : L'Harmattan.

PAQUAY L. (2006). "Au-delà des cloisonnements entre divers types de recherche, quels critères de qualité ? ». In L. Paquay, M. Crahay \& J.-M. De Ketele (dir.), L'analyse qualitative en éducation. Des pratiques de recherche aux critères de qualité. Bruxelles : De Boeck, p. 13-31.

PEREZ-ROUX T. (2008). "Évolution des représentations et des pratiques dans la formation initiale des enseignants : éléments de construction d'une identité professionnelle à l'échelle du temps ». Éducation et formation, no 287, p.7-19.

PERRENOUD P. (1983) « La pratique pédagogique entre l'improvisation réglée et le bricolage ». Éducation et recherche, n० 2, p. 198-212

RATHS J. (2001). "Teachers' beliefs and teaching beliefs ». Early Childhood Research and Practice, vol. 3, no 1, p. 385-391.

RICHARDSON V. (1994). "The consideration of beliefs in staff development ". In V. Richardson, Teacher change and the staff development process: A case of reading instruction. New York: Teachers College Press, p. 90-108.

RICHARDSON V. (1996). "The role of attitudes and beliefs in learning to teach ". In J. Sikula, Handbook of Research on Teacher Education. New York : Macmillan, p. 102-119.

RICHARDSON V. \& HAMILTON M. L. (1994). "The practical argument staff development process". In V. Richardson, Teacher change and staff development process : A case in reading instruction. New York : Teachers College Press, p. 109-134.

RICHARDSON V. \& PLACIER P. (2001). "Teacher change ». In V. Richardson, Handbook of research on teaching. Washington : American educational research association, p. 905947.

SHAPIRO B. L. (1991). "A collaborative approach to help novice science teachers reflect on changes in their construction of the role of science teacher ". Alberta Journal of Educational Research, vol. 37, p. 119-132.

SKINNER B. F. (1968). The Technology of Teaching. New York : Appleton Century Crofts. 
TANASE M. \& WANG J. (2010). "Initial epistemological beliefs transformation in one teacher education classroom: Case study of four preservice teachers ". Teaching and teacher Education, vol. 26, p. 1238-1248.

TILLEMA H. \& KNOL W. (1997). "Promoting student teacher learning through conceptual change or direct instruction ". Teaching and teacher Éducation, vol. 13, no 6, p. 579-595.

VANDERBERGHE R. (2006). "La recherche qualitative en éducation : dégager le sens et démêler la complexité ". In L. Paquay, M. Crahay \& J.-M. De Ketele (dir.), L'analyse qualitative en éducation. Des pratiques de recherche aux critères de qualité. Bruxelles : De Boeck, p. 53-65.

VAN NIEUWENHOVEN C. \& LABEEU M. (2010). «L'accompagnement par le superviseur lors de l'entretien à chaud dans un curriculum à visée réflexive ". Éducation et francophonie, vol. 38, n० 2, p. 39-59.

VANHULLE S. (2009). "Les savoirs professionnels se construisent dans un monde intermédiaire ". Prismes : revue pédagogique HEP, n० 10, p. 20-22.

VEENMAN S. (1984). "Perceived problems of beginning teachers ". Review of Educational Research, vol. 54, no 2, p. 143-177.

VERLOOP N., VAN DRIEL J. \& MEIJER P. (2001). "Teacher knowledge and the knowledge base of teaching ". International Journal of Educational Research, vol. 35 , no 5 , p. 441461.

WEINSTEIN C. (1989). "Teacher education students' preconceptions of teaching ». Journal of Teacher Education, vol. 40, n० 2, p. 53-60.

WILCOX S., SHRAM P., LAPPAN G. \& LANIER P. (1991). The role of a learning community in changing preservice teachers' knowledge. East Lansing (États-Unis) : National Center for Research on Teacher Learning, College of Education, Michigan State University.

WOOLFOLK HOY A. \& BURKE SPERO R. (2005). "Changes in teacher efficacy during the early years of teaching: A Comparison of four measures ". Teaching and Teacher Education, no 21, p. 343-356.

WOOLFOLK HOY A., DAVIS H. \& PAPE S. (2006). "Teachers' knowledge and beliefs ". In P. A. Alexander \& P. H. Winne, Handbook of educational psychology ( $2^{\mathrm{e}}$ éd.). Mahwah (États-Unis) : Lawrence Erlbaum, p. 715-737. 


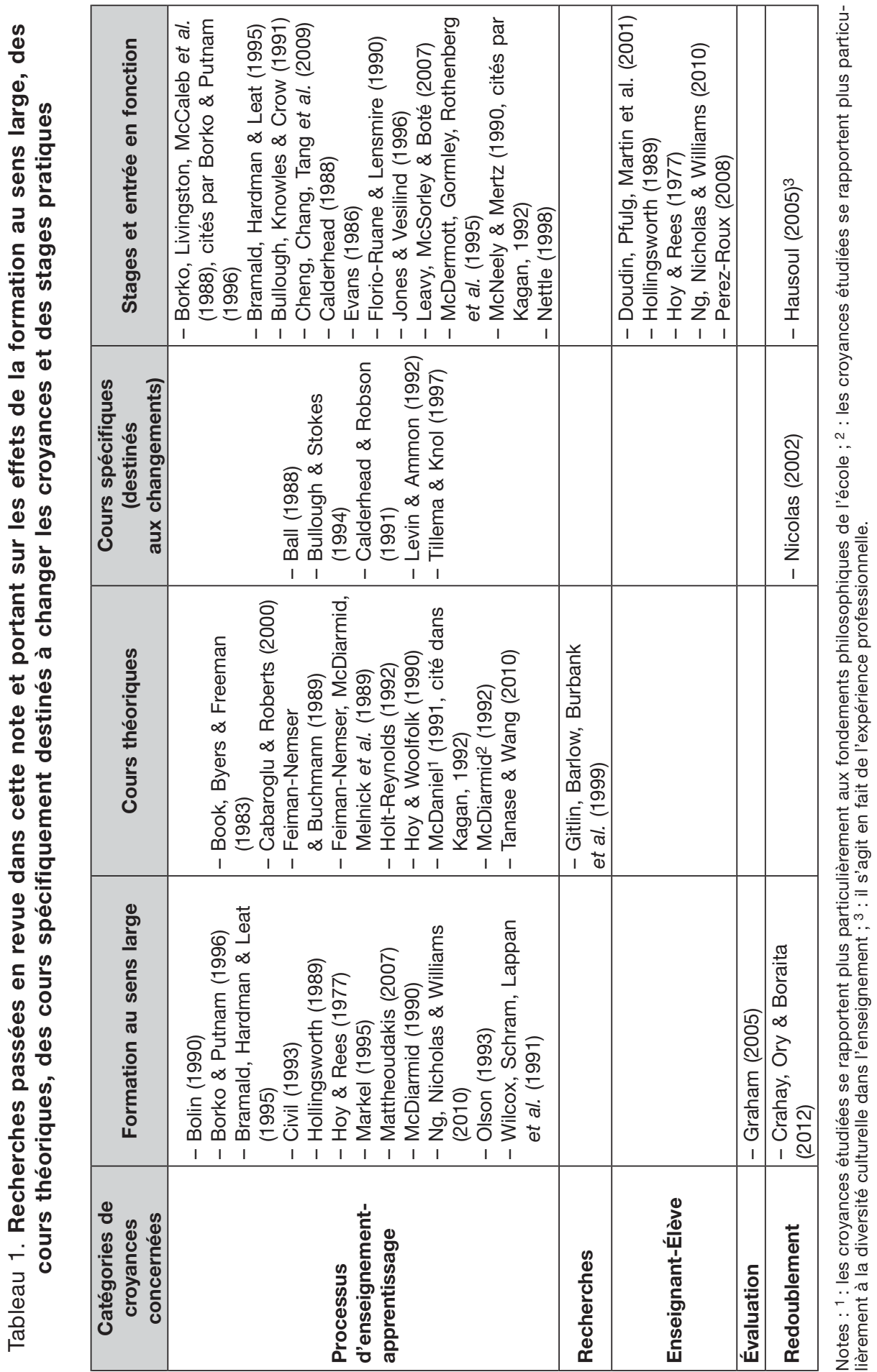




\begin{tabular}{|c|c|c|c|c|c|c|}
\hline 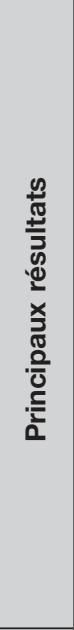 & 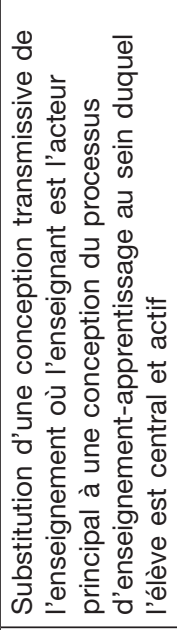 & 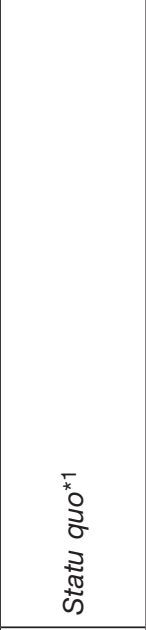 & 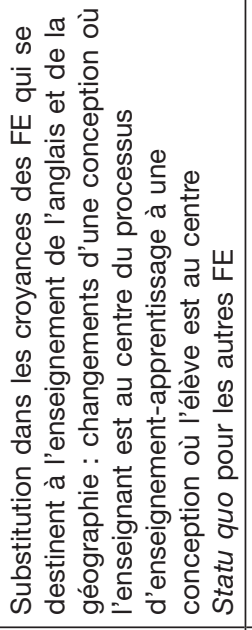 & 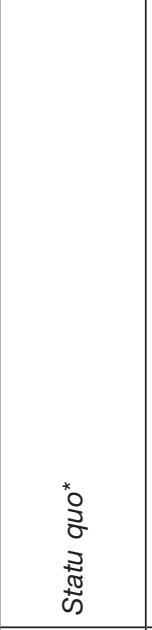 & 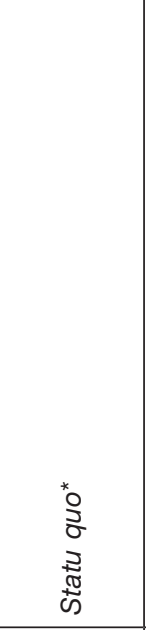 & 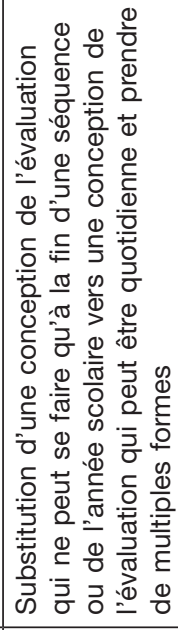 \\
\hline 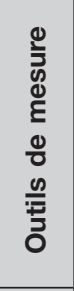 & 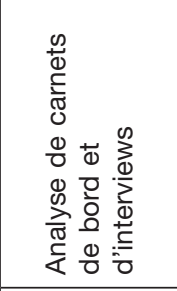 & 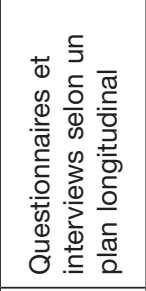 & 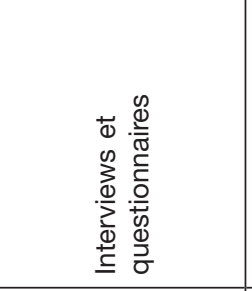 & 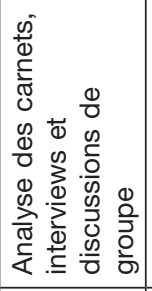 & 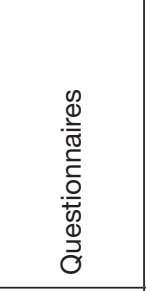 & 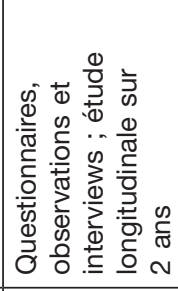 \\
\hline 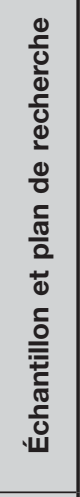 & 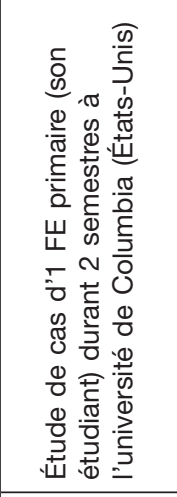 & 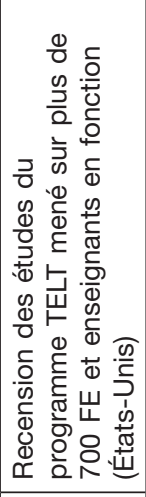 & 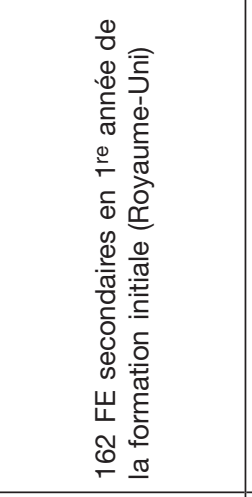 & 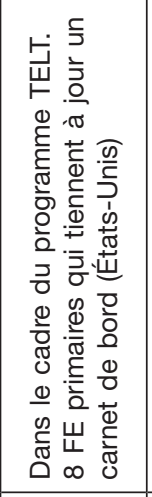 & 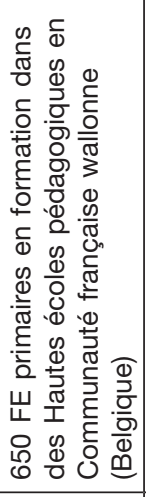 & 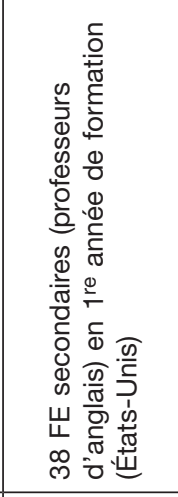 \\
\hline 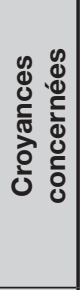 & 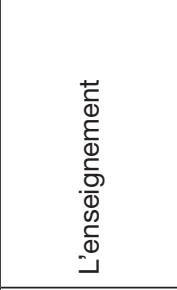 & 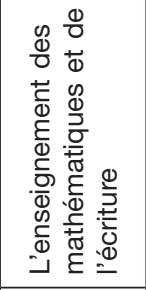 & 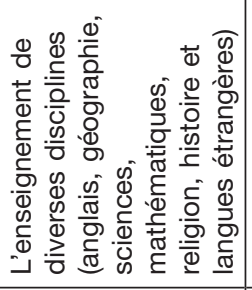 & 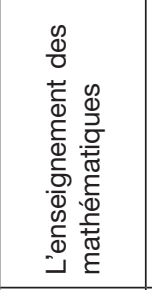 & 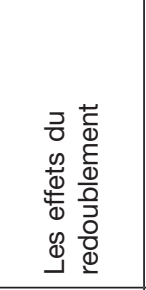 & 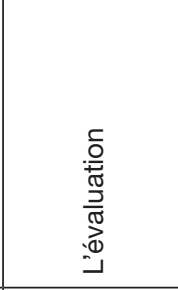 \\
\hline$\frac{\infty}{5}$ & 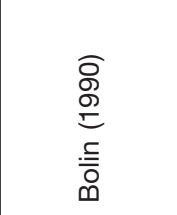 & 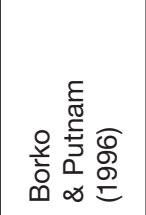 & 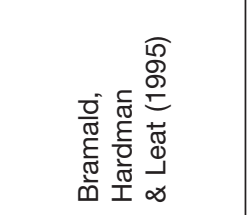 & 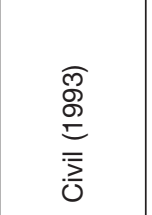 & 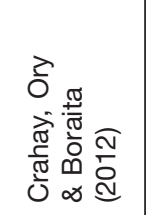 & 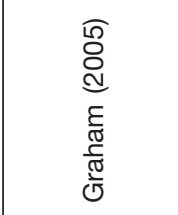 \\
\hline
\end{tabular}




\begin{tabular}{|c|c|c|c|c|c|}
\hline 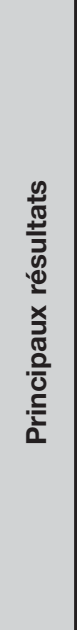 & 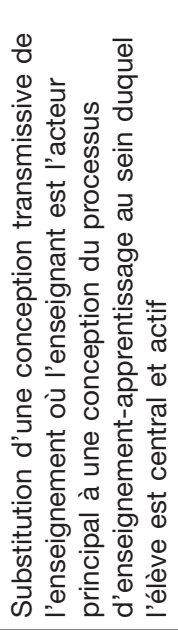 & 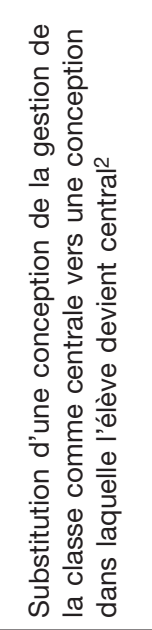 & 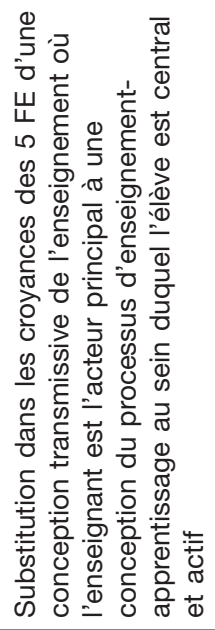 & 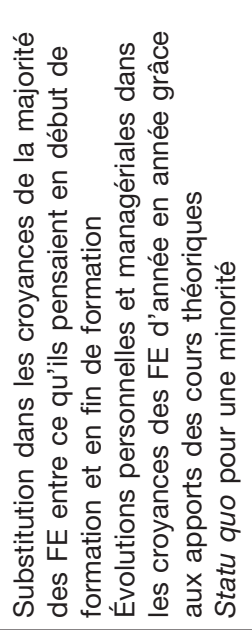 & 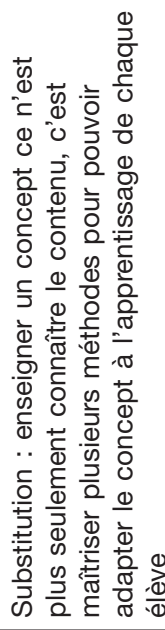 \\
\hline 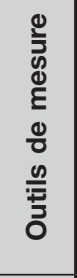 & 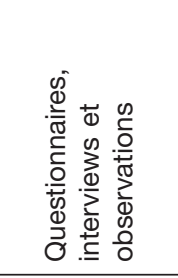 & 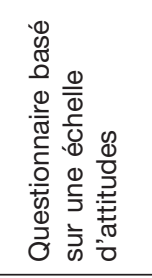 & 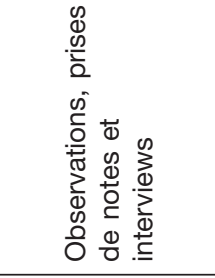 & 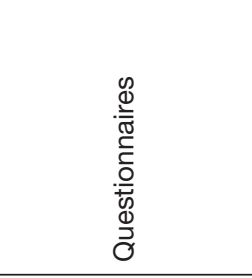 & 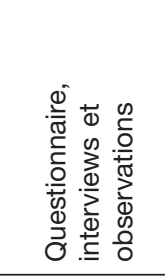 \\
\hline 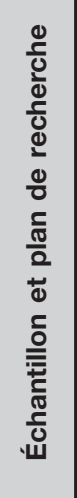 & 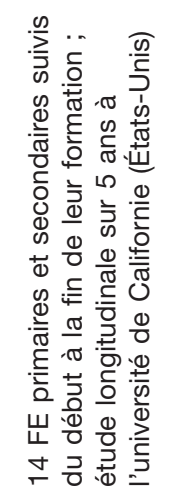 & 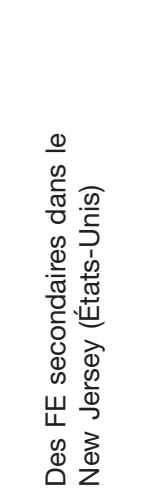 & 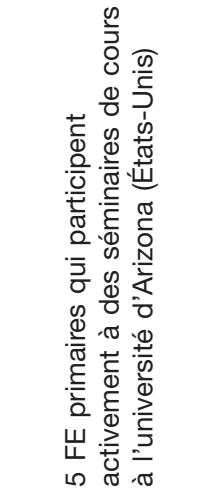 & 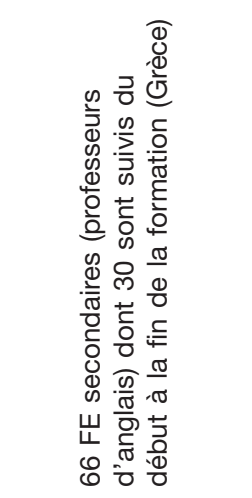 & 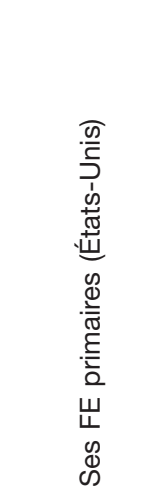 \\
\hline 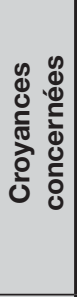 & 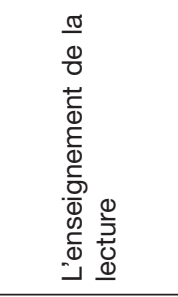 & 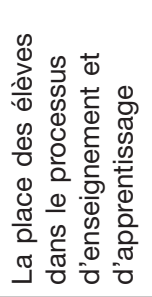 & 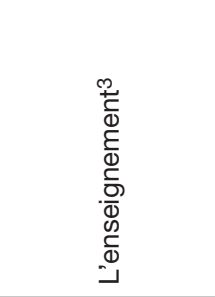 & 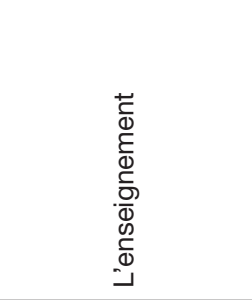 & 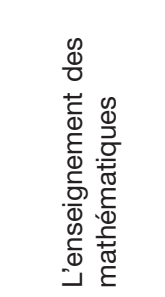 \\
\hline 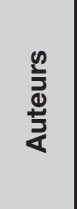 & 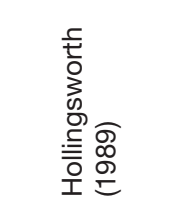 & $\begin{array}{l}\mathscr{0} \\
\mathbb{0} \\
\simeq \\
\infty \\
\infty \\
\text { 옹 } \\
\text { 옫 }\end{array}$ & 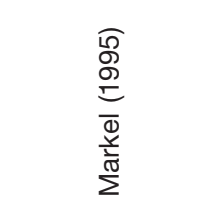 & 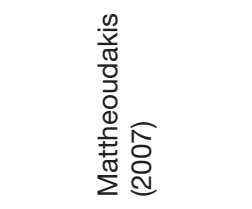 & 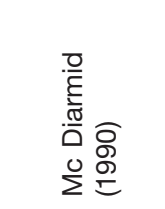 \\
\hline
\end{tabular}




\begin{tabular}{|c|c|c|c|c|}
\hline 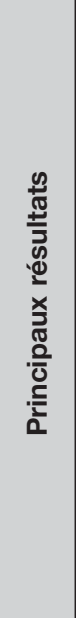 & 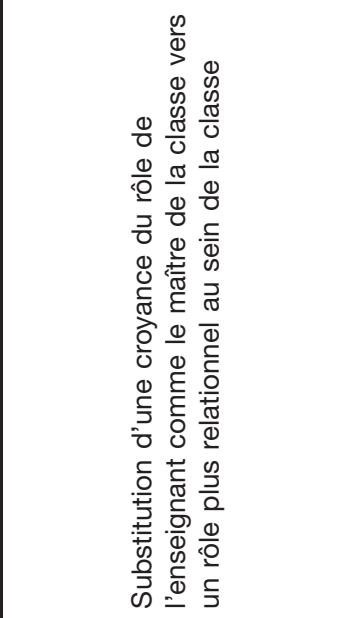 & 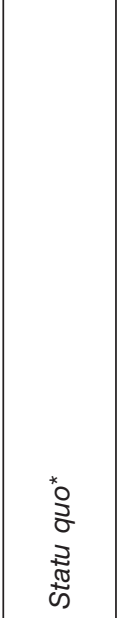 & 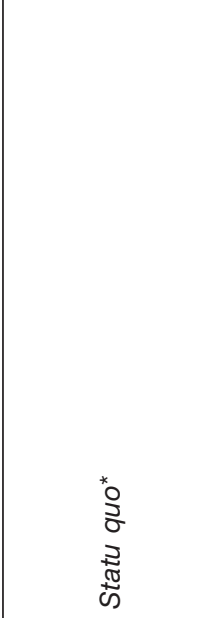 & 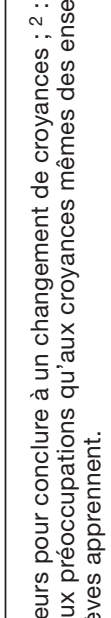 \\
\hline 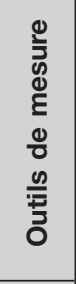 & 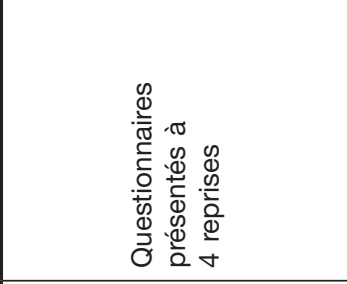 & 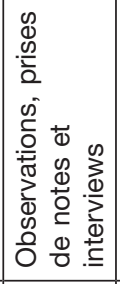 & 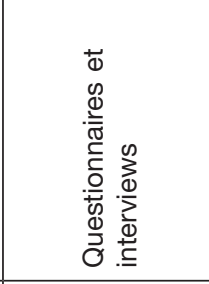 & 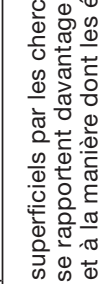 \\
\hline 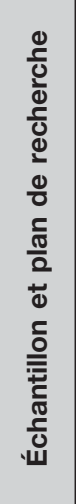 & 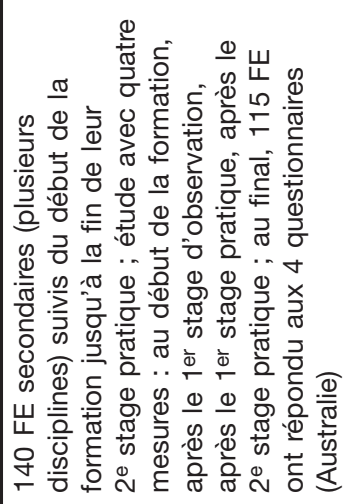 & 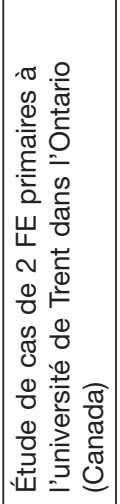 & 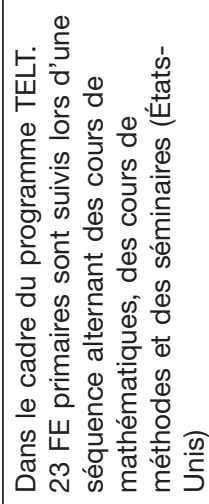 & 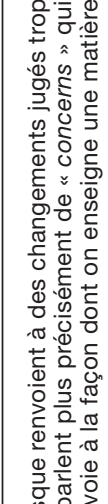 \\
\hline 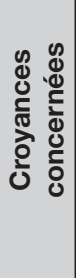 & 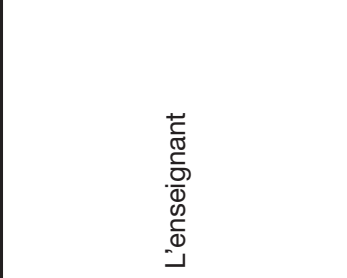 & 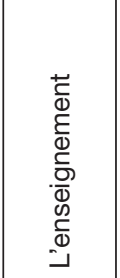 & 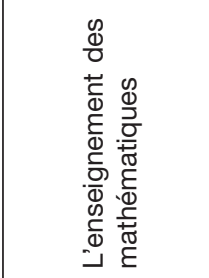 & 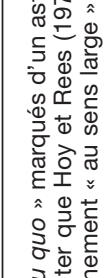 \\
\hline $\begin{array}{l}\frac{\infty}{3} \\
\frac{0}{2} \\
\frac{1}{3}\end{array}$ & 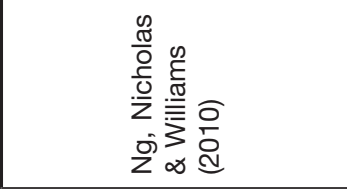 & 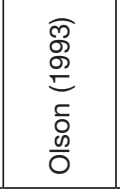 & 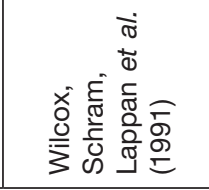 & 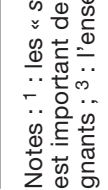 \\
\hline
\end{tabular}




\begin{tabular}{|c|c|c|c|c|}
\hline 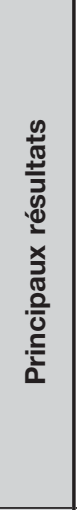 & 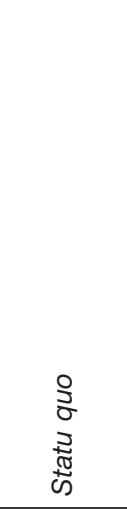 & 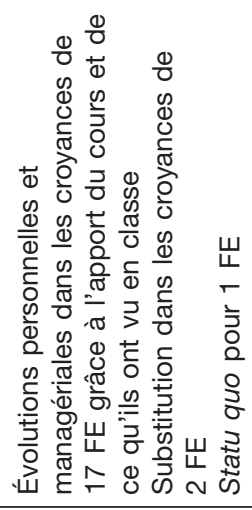 & 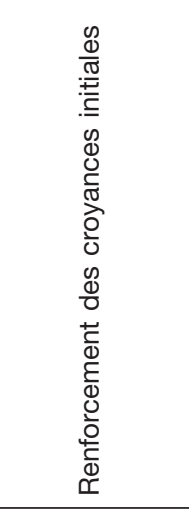 & 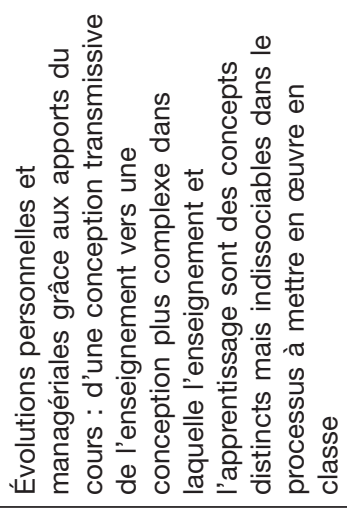 \\
\hline 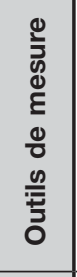 & 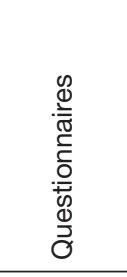 & : & 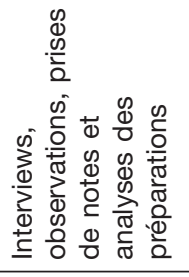 & 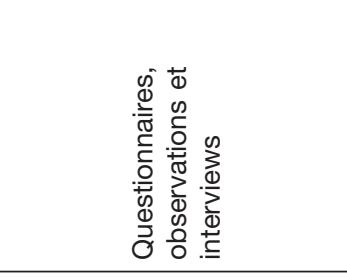 \\
\hline 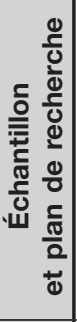 & 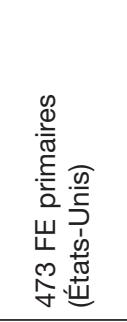 & 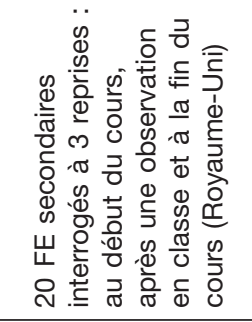 & 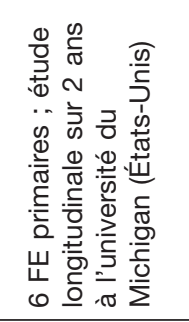 & 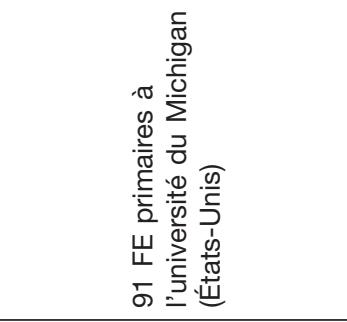 \\
\hline 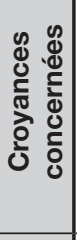 & 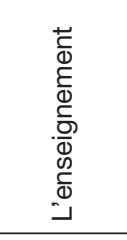 & 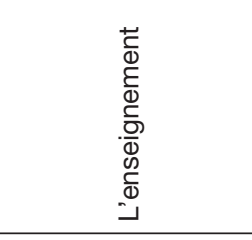 & 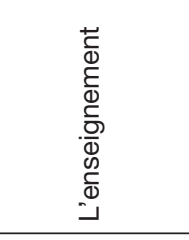 & 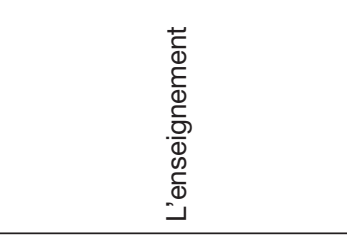 \\
\hline 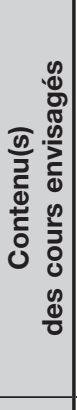 & 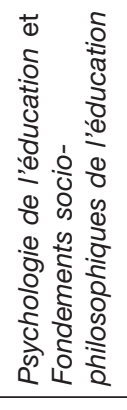 & 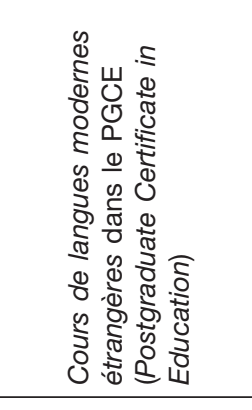 & 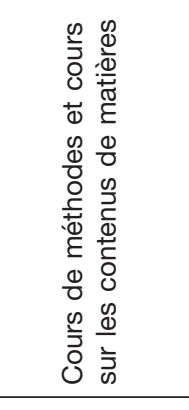 & 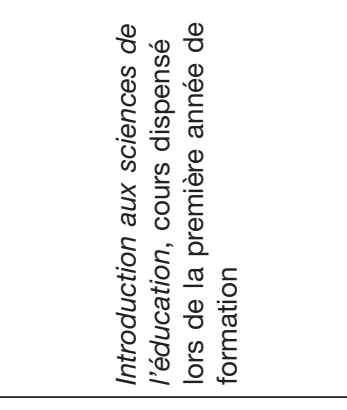 \\
\hline 足 & 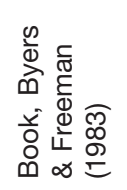 & 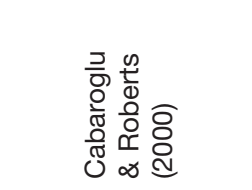 & 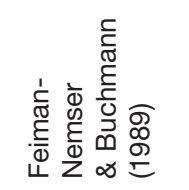 & 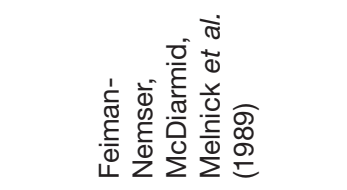 \\
\hline
\end{tabular}




\begin{tabular}{|c|c|c|c|c|c|c|}
\hline 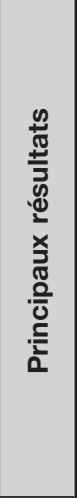 & 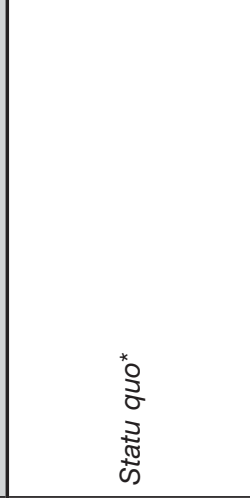 & $\begin{array}{l}* 0 \\
0 \\
0 \\
3 \\
\frac{\pi}{0} \\
\omega \\
\end{array}$ & 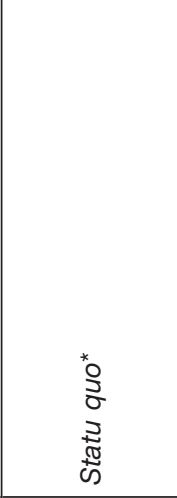 & 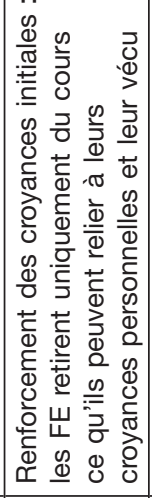 & 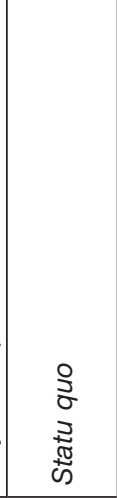 & 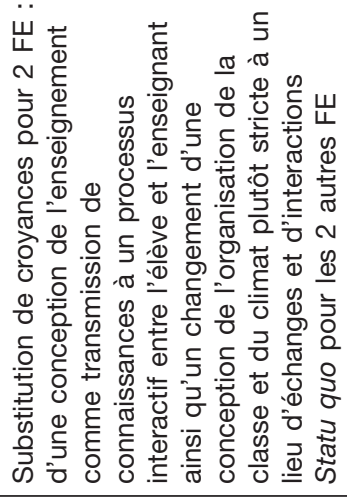 \\
\hline 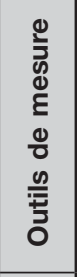 & 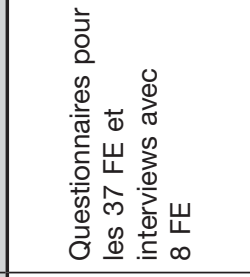 & 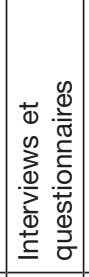 & 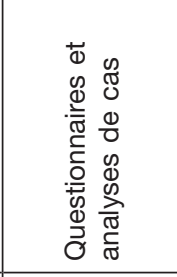 & 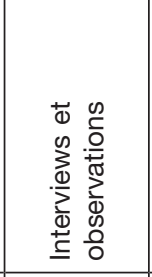 & 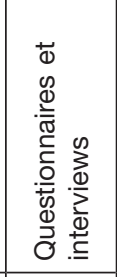 & 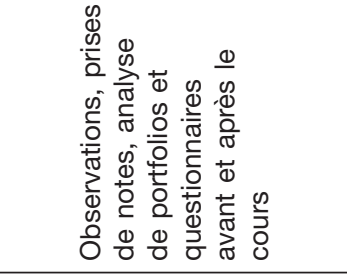 \\
\hline 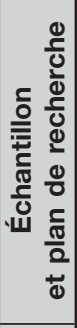 & 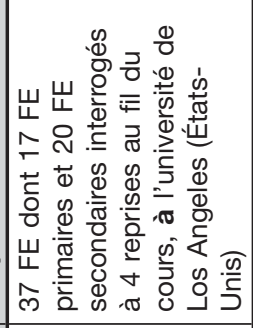 & 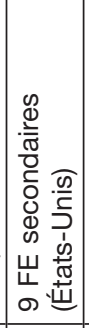 & 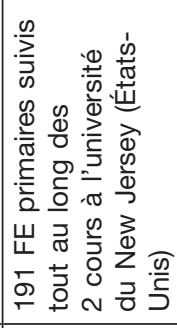 & 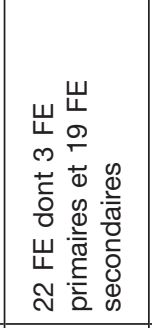 & 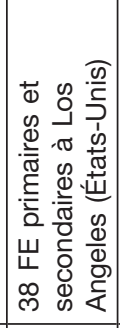 & 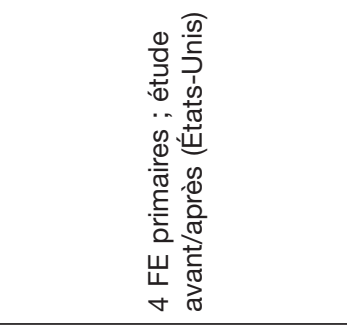 \\
\hline 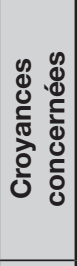 & 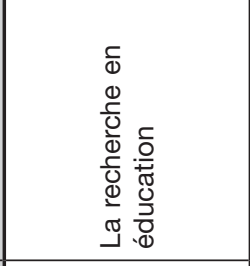 & 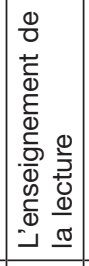 & 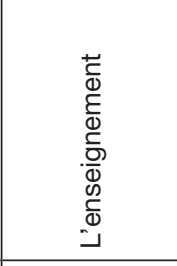 & 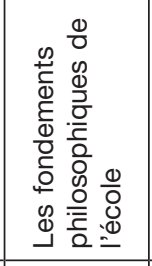 & 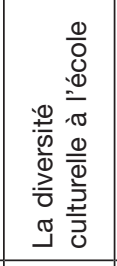 & 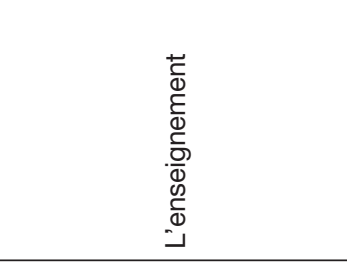 \\
\hline 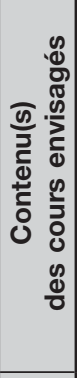 & 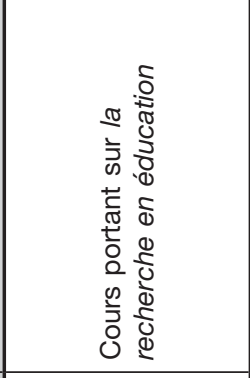 & 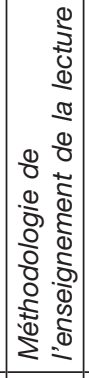 & 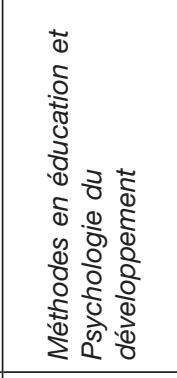 & 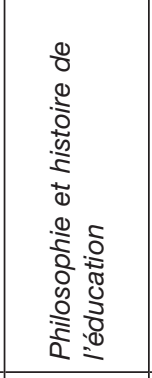 & 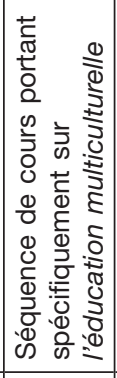 & 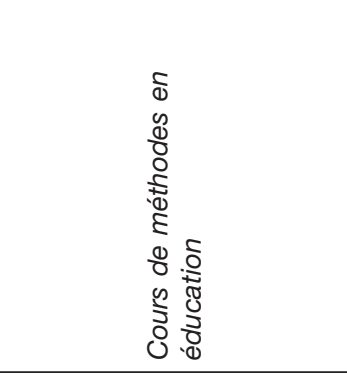 \\
\hline 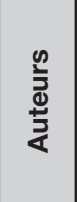 & 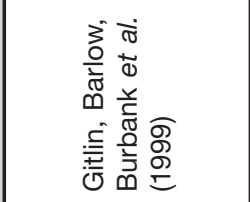 & 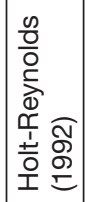 & 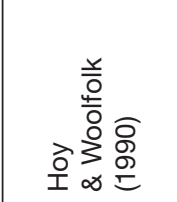 & 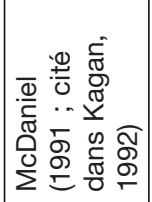 & 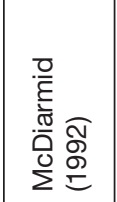 & 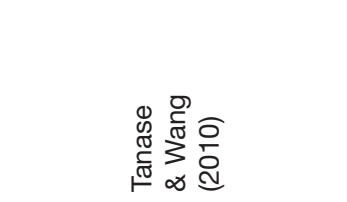 \\
\hline
\end{tabular}




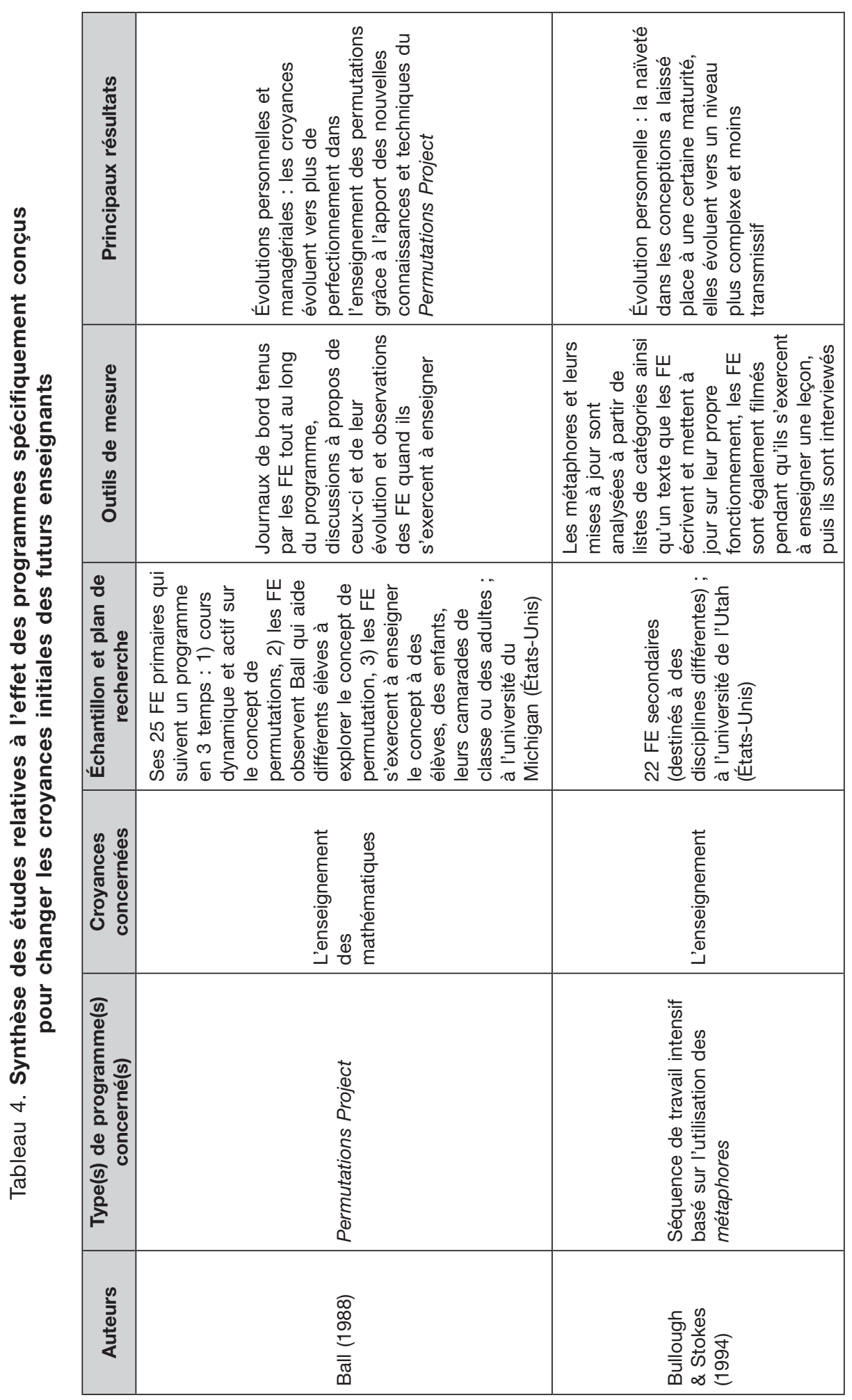




\begin{tabular}{|c|c|c|c|c|}
\hline 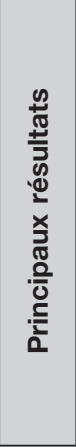 & 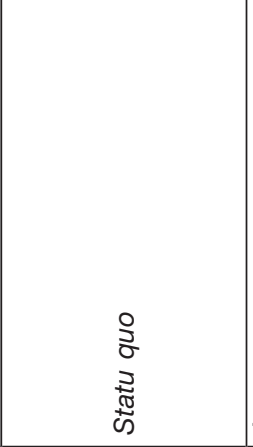 & 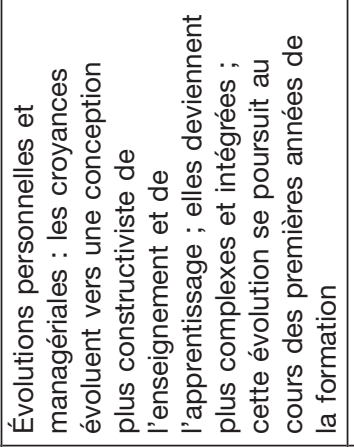 & 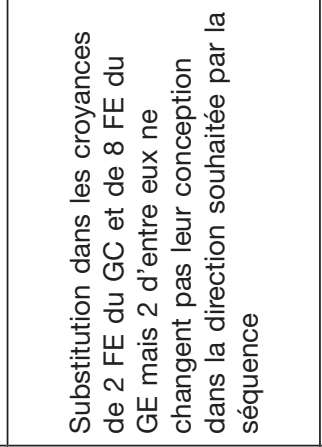 & 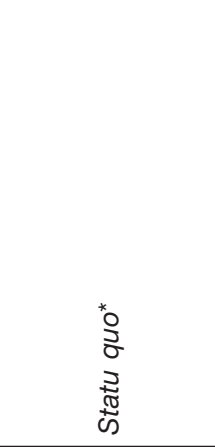 \\
\hline 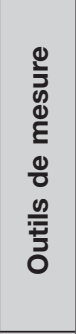 & 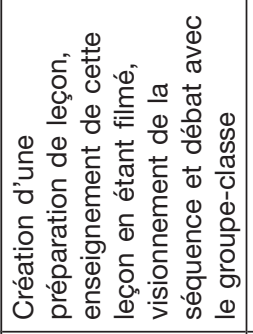 & 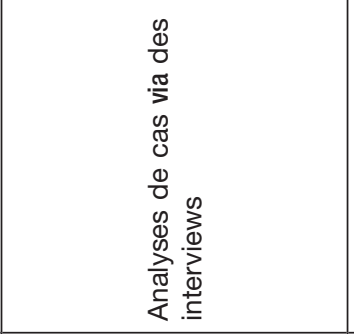 & 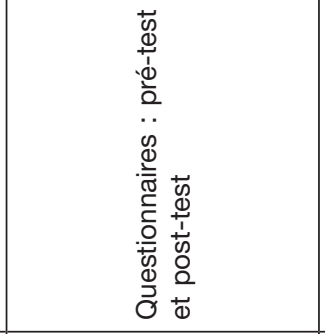 & 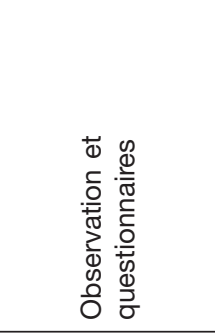 \\
\hline 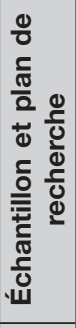 & 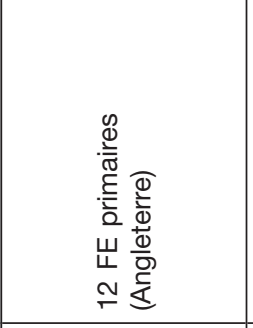 & 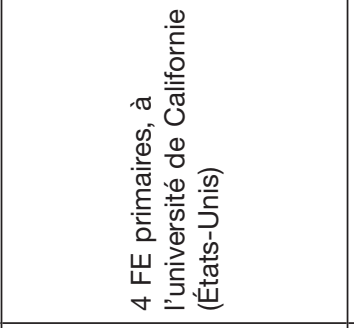 & 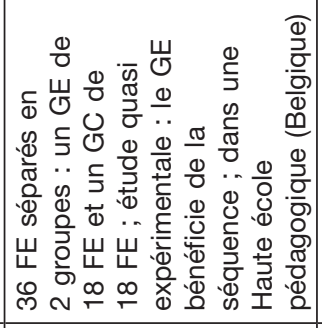 & 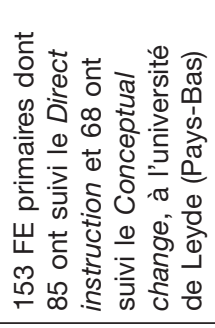 \\
\hline 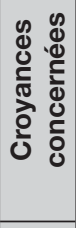 & 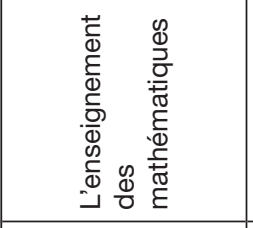 & 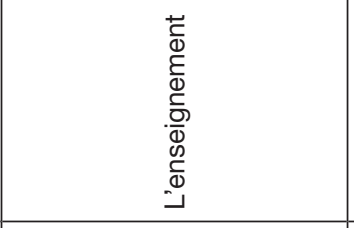 & 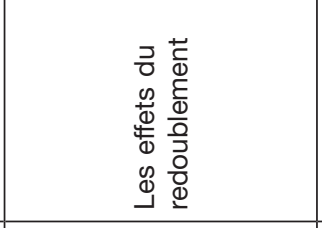 & 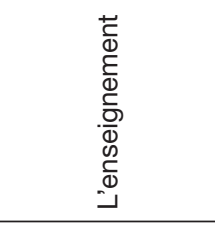 \\
\hline 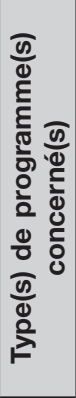 & 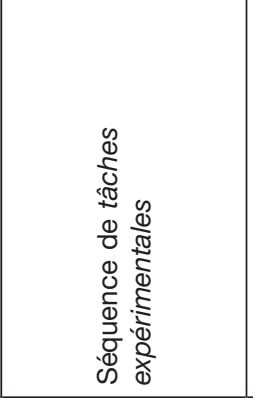 & 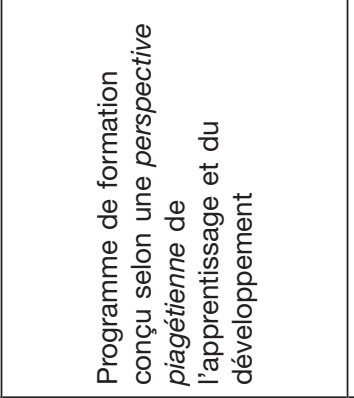 & 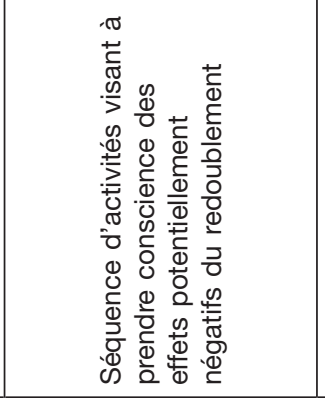 & 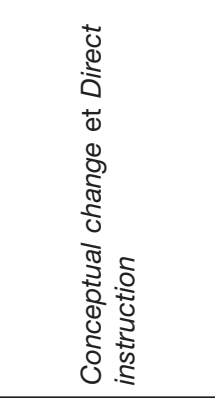 \\
\hline 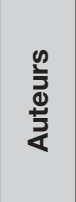 & 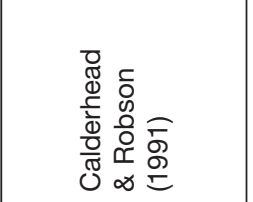 & 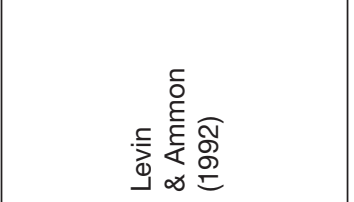 & 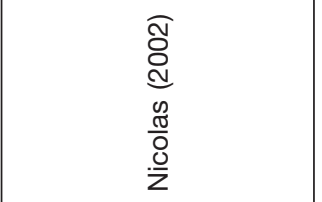 & 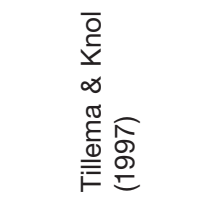 \\
\hline
\end{tabular}




\begin{tabular}{|c|c|c|c|c|}
\hline 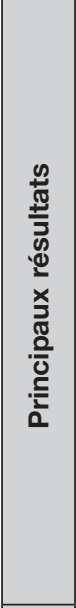 & 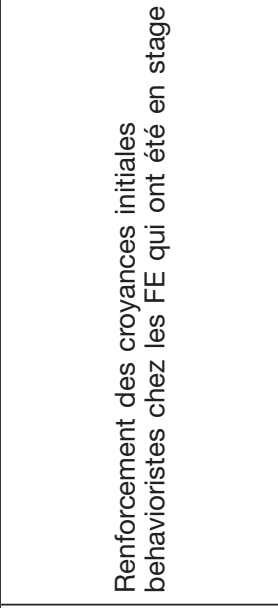 & 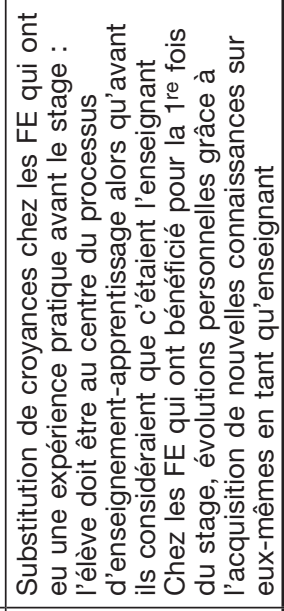 & 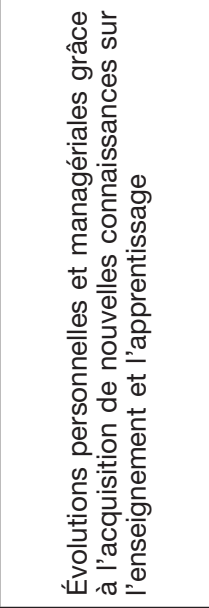 & 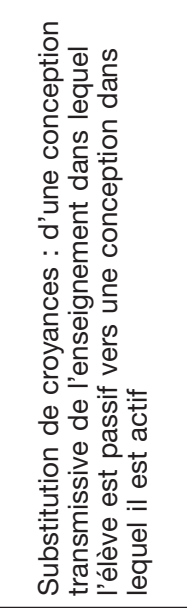 \\
\hline 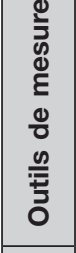 & 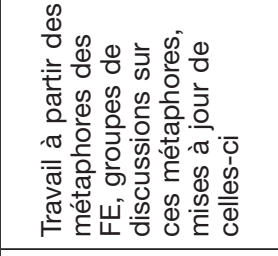 & 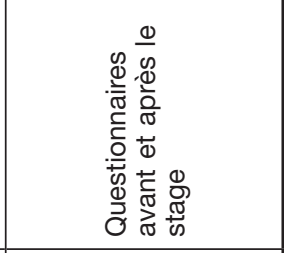 & 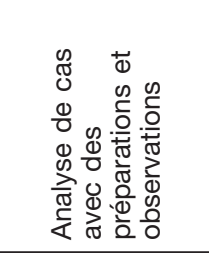 & 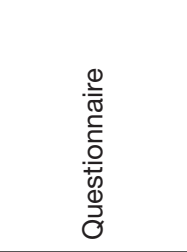 \\
\hline 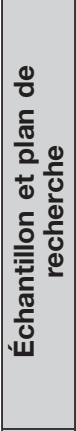 & 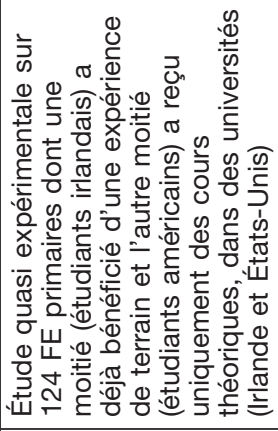 & 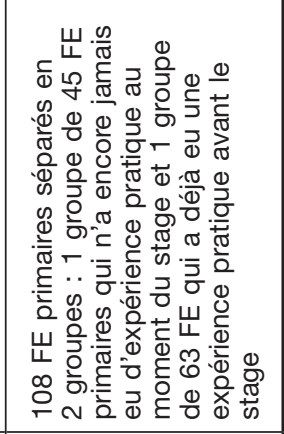 & 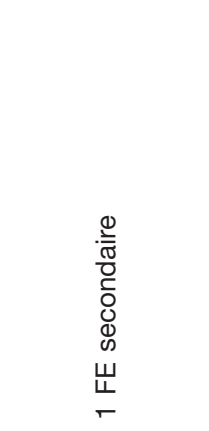 & 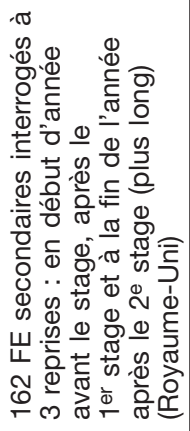 \\
\hline 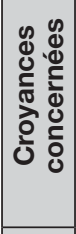 & 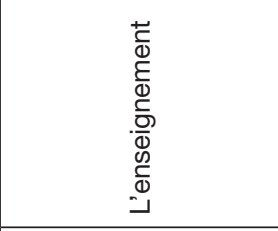 & 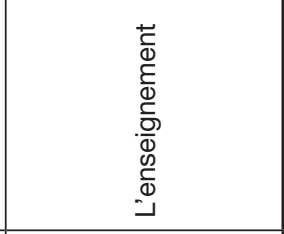 & 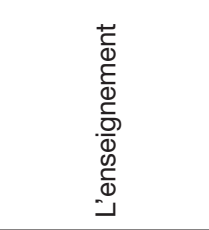 & 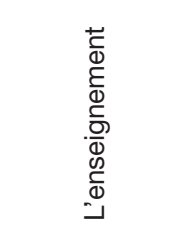 \\
\hline 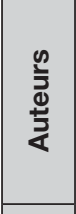 & 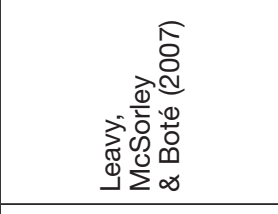 & 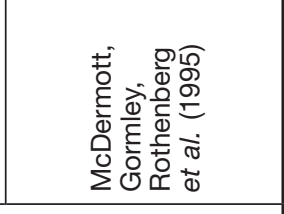 & 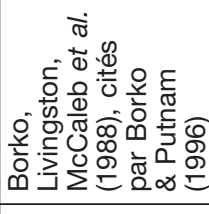 & 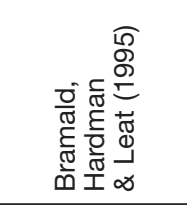 \\
\hline 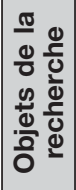 & \multicolumn{2}{|c|}{ 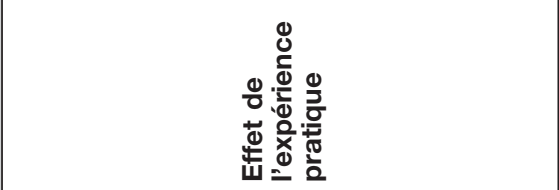 } & \multicolumn{2}{|c|}{ 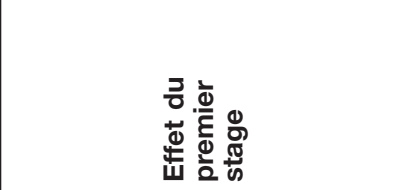 } \\
\hline
\end{tabular}




\begin{tabular}{|c|c|c|c|c|c|}
\hline 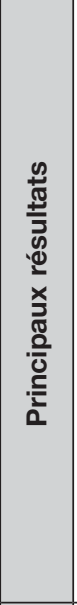 & 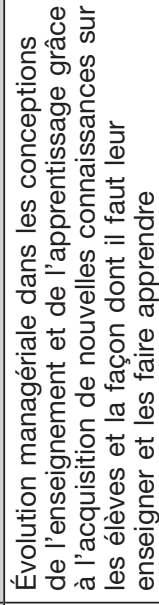 & 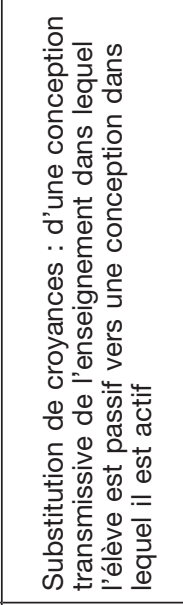 & 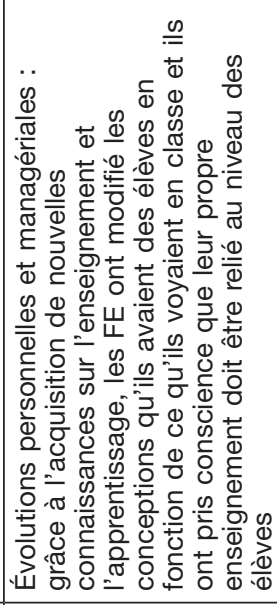 & 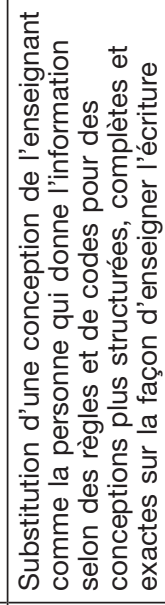 & 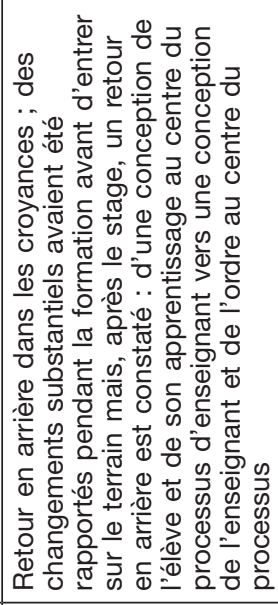 \\
\hline 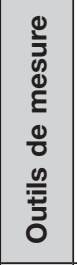 & 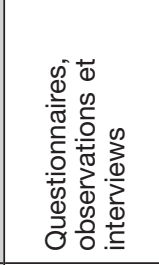 & 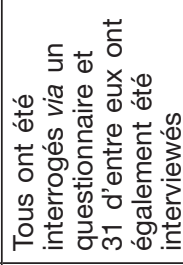 & 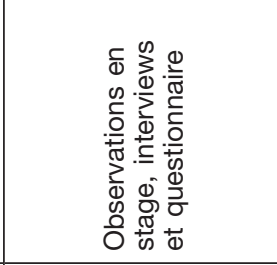 & 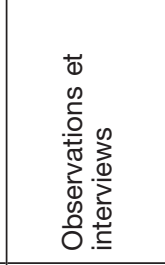 & 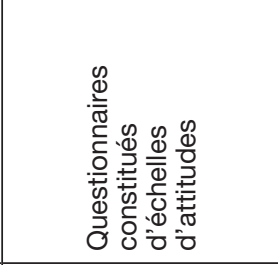 \\
\hline 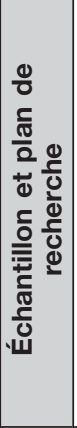 & 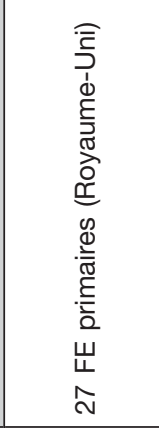 & 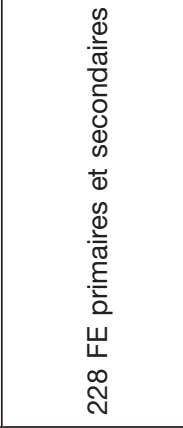 & 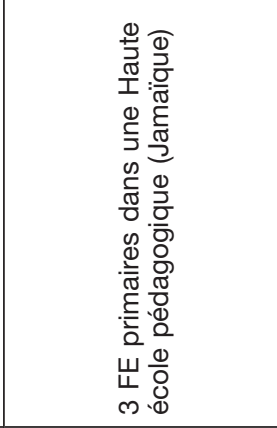 & 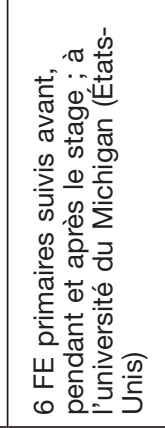 & 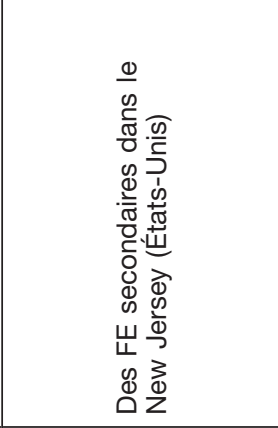 \\
\hline 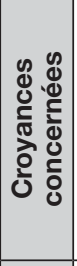 & 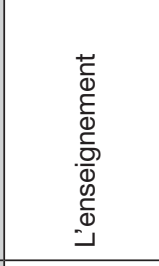 & 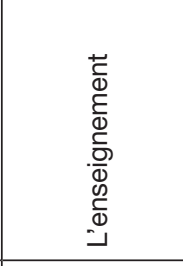 & 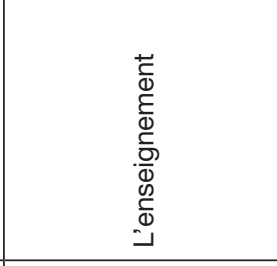 & 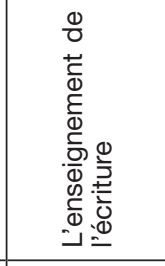 & 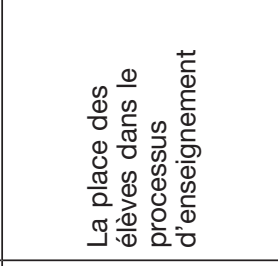 \\
\hline 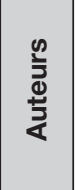 & 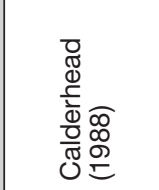 & 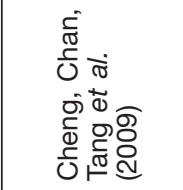 & 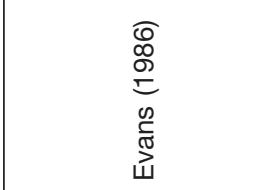 & 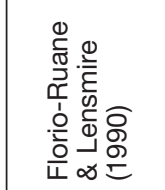 & 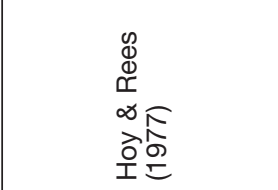 \\
\hline 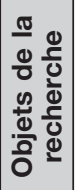 & & & 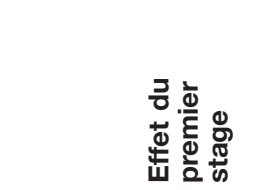 & & \\
\hline
\end{tabular}




\begin{tabular}{|c|c|c|c|c|c|}
\hline 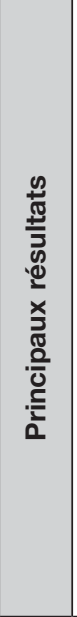 & 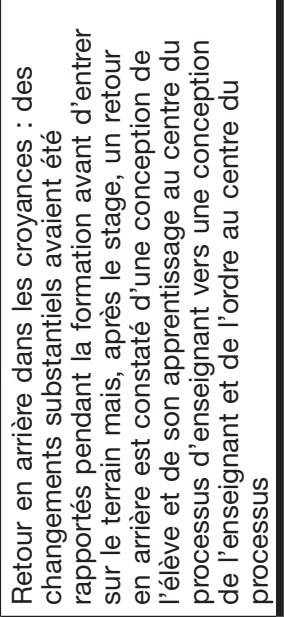 & 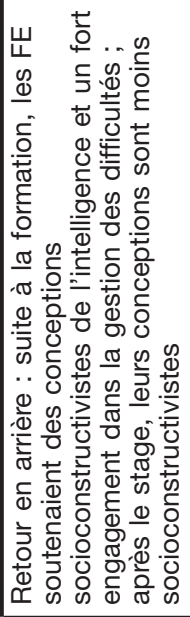 & 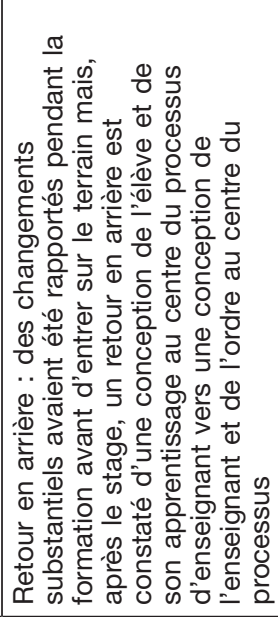 & 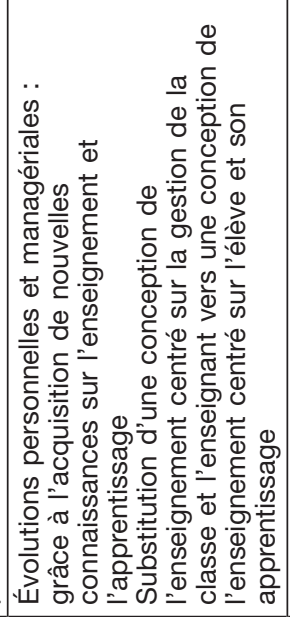 & 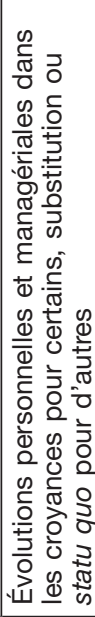 \\
\hline 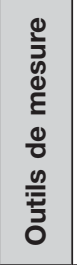 & 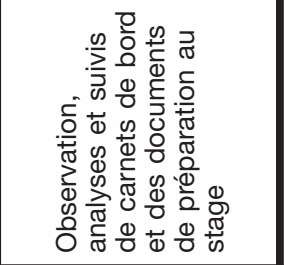 & 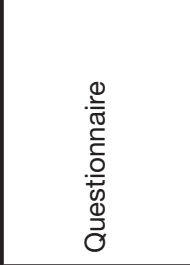 & 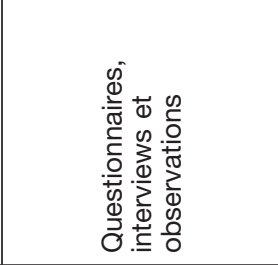 & 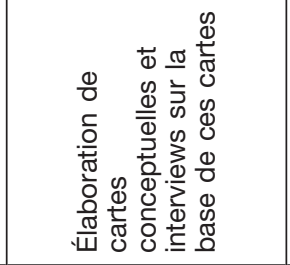 & 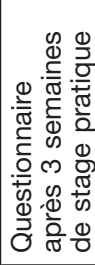 \\
\hline 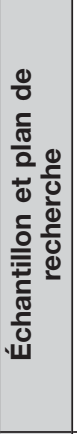 & 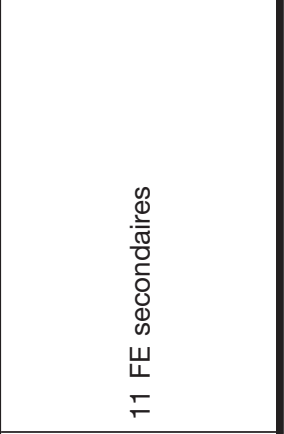 & 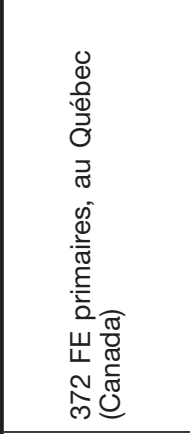 & 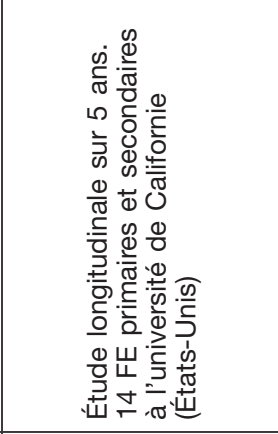 & 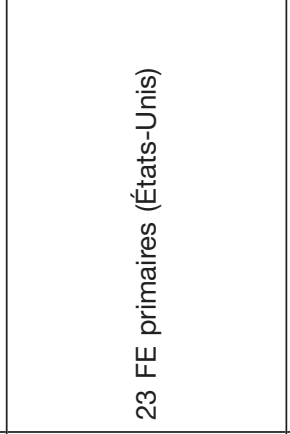 & 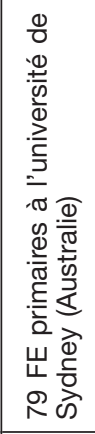 \\
\hline 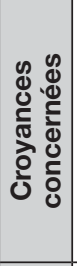 & 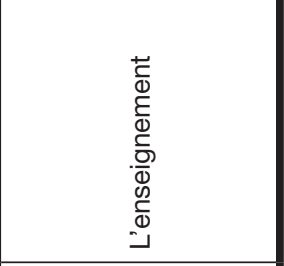 & 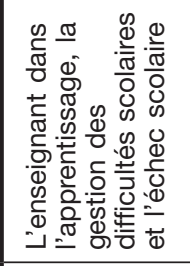 & 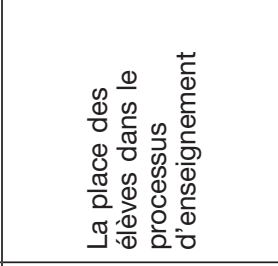 & 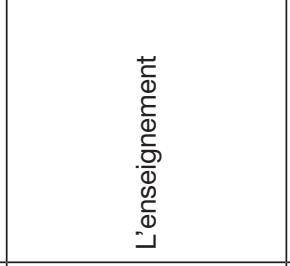 & 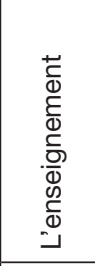 \\
\hline 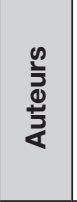 & 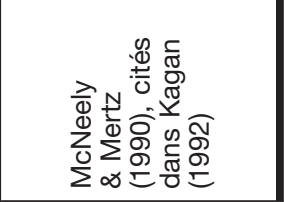 & 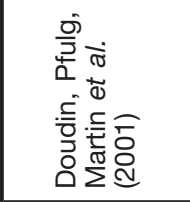 & 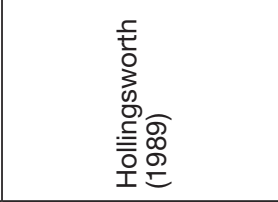 & 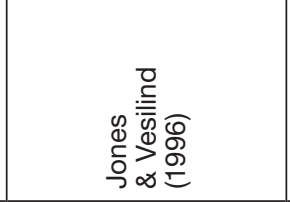 & 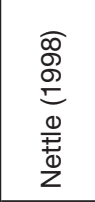 \\
\hline 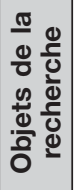 & 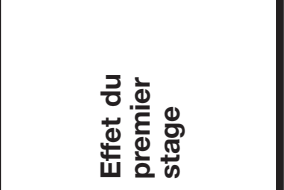 & & 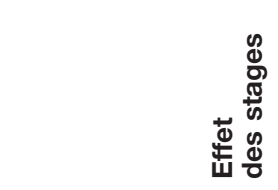 & & \\
\hline
\end{tabular}




\begin{tabular}{|c|c|c|c|c|}
\hline 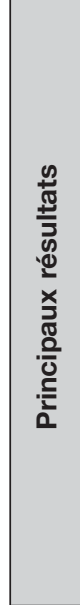 & 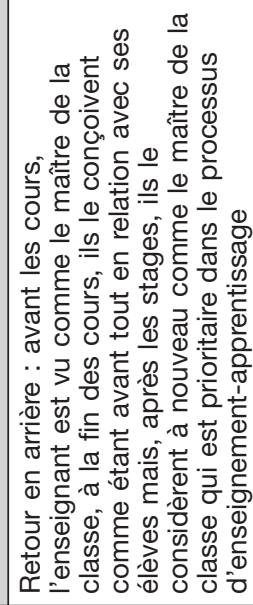 & 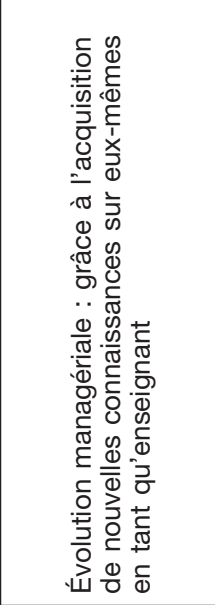 & 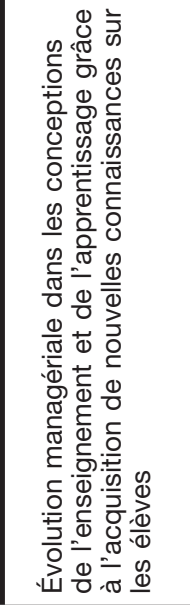 & 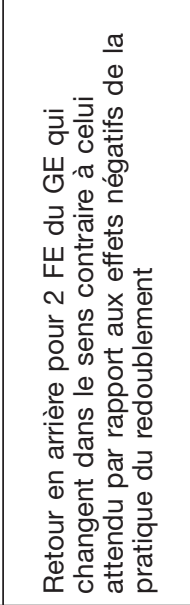 \\
\hline 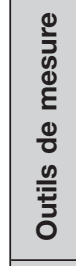 & 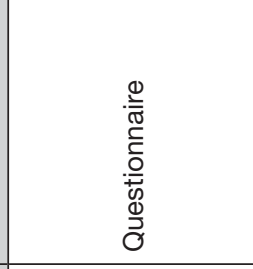 & 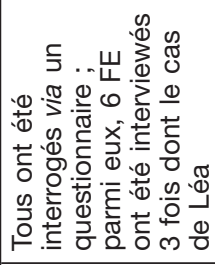 & 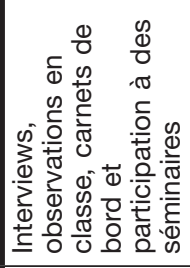 & $\begin{array}{l}\sum_{0}^{0} \\
\substack{0 \\
0} \\
\stackrel{0}{ \pm}\end{array}$ \\
\hline 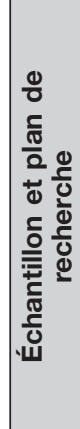 & 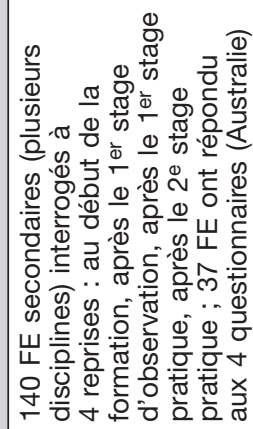 & 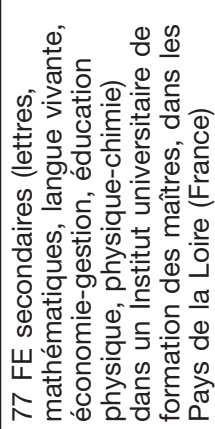 & 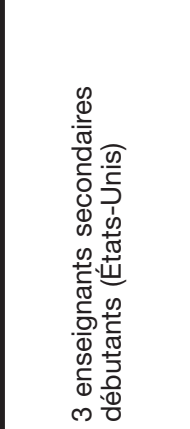 & 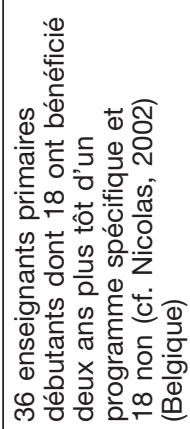 \\
\hline 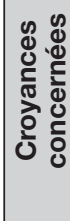 & 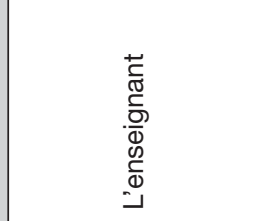 & 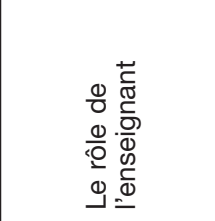 & 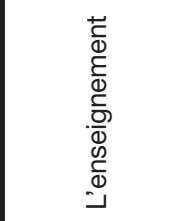 & 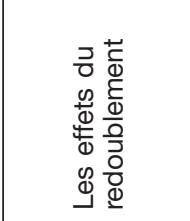 \\
\hline 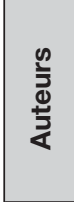 & 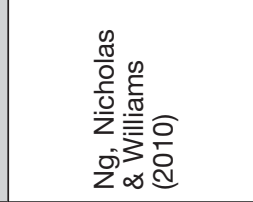 & 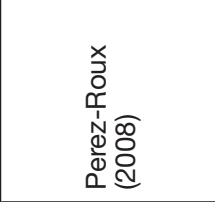 & 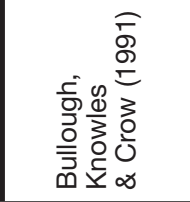 & 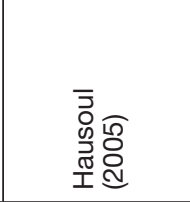 \\
\hline 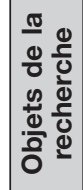 & \multicolumn{2}{|c|}{ 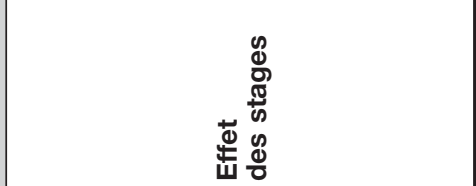 } & \multicolumn{2}{|c|}{ 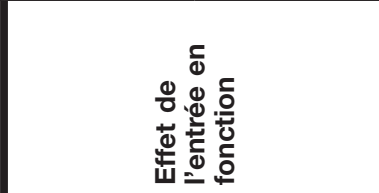 } \\
\hline
\end{tabular}




\begin{tabular}{|c|c|c|c|}
\hline 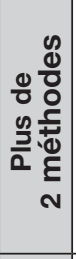 & 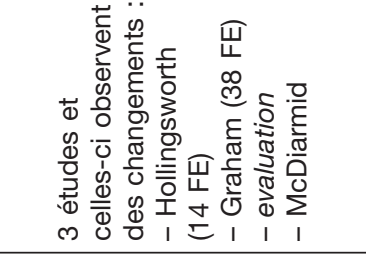 & 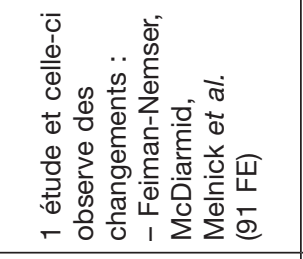 & - \\
\hline 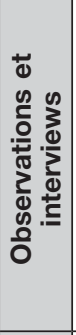 & 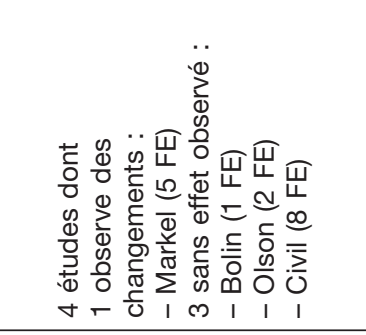 & 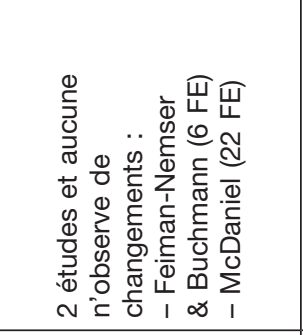 & 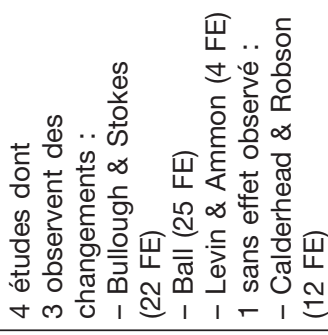 \\
\hline 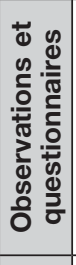 & 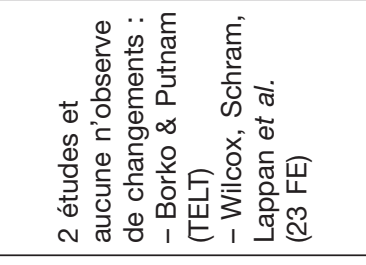 & 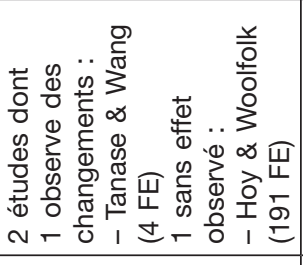 & - \\
\hline 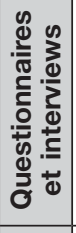 & 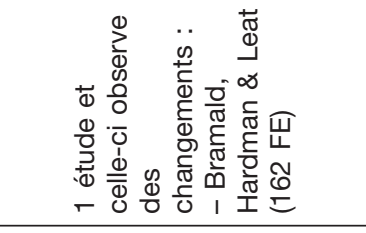 & 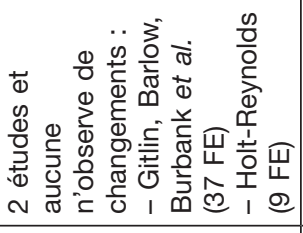 & 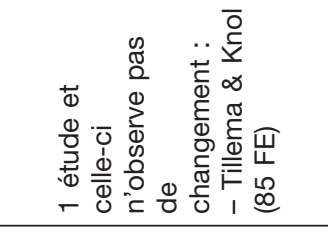 \\
\hline 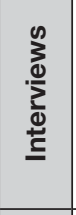 & - & 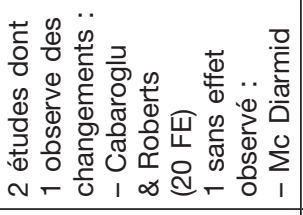 & - \\
\hline 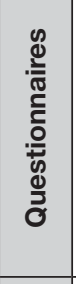 & 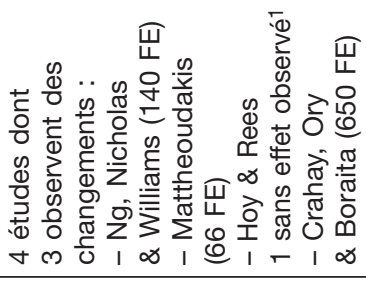 & 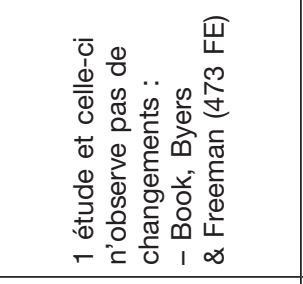 & 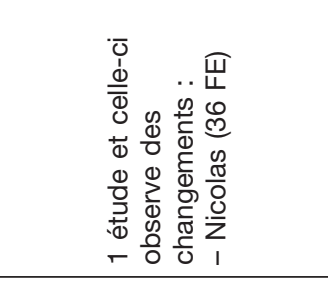 \\
\hline & 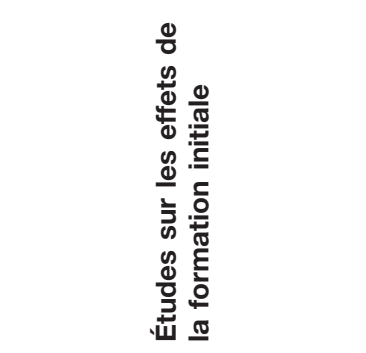 & 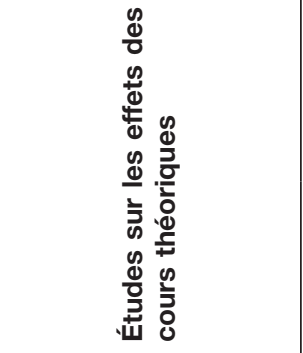 & 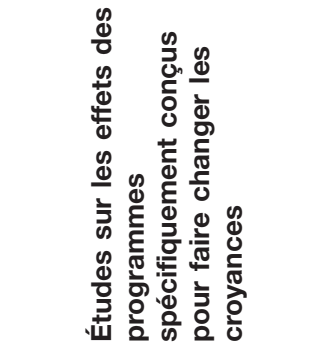 \\
\hline
\end{tabular}




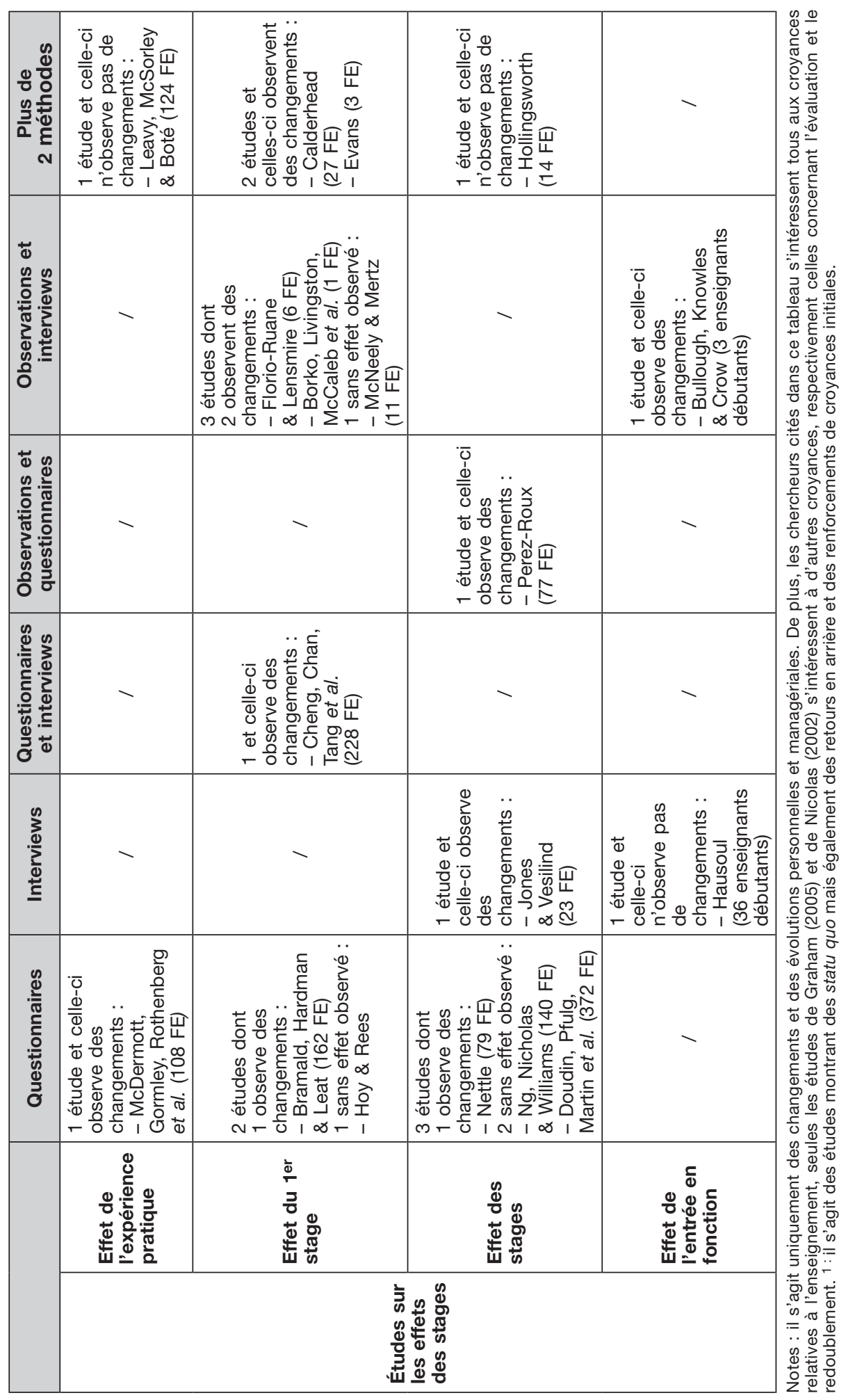




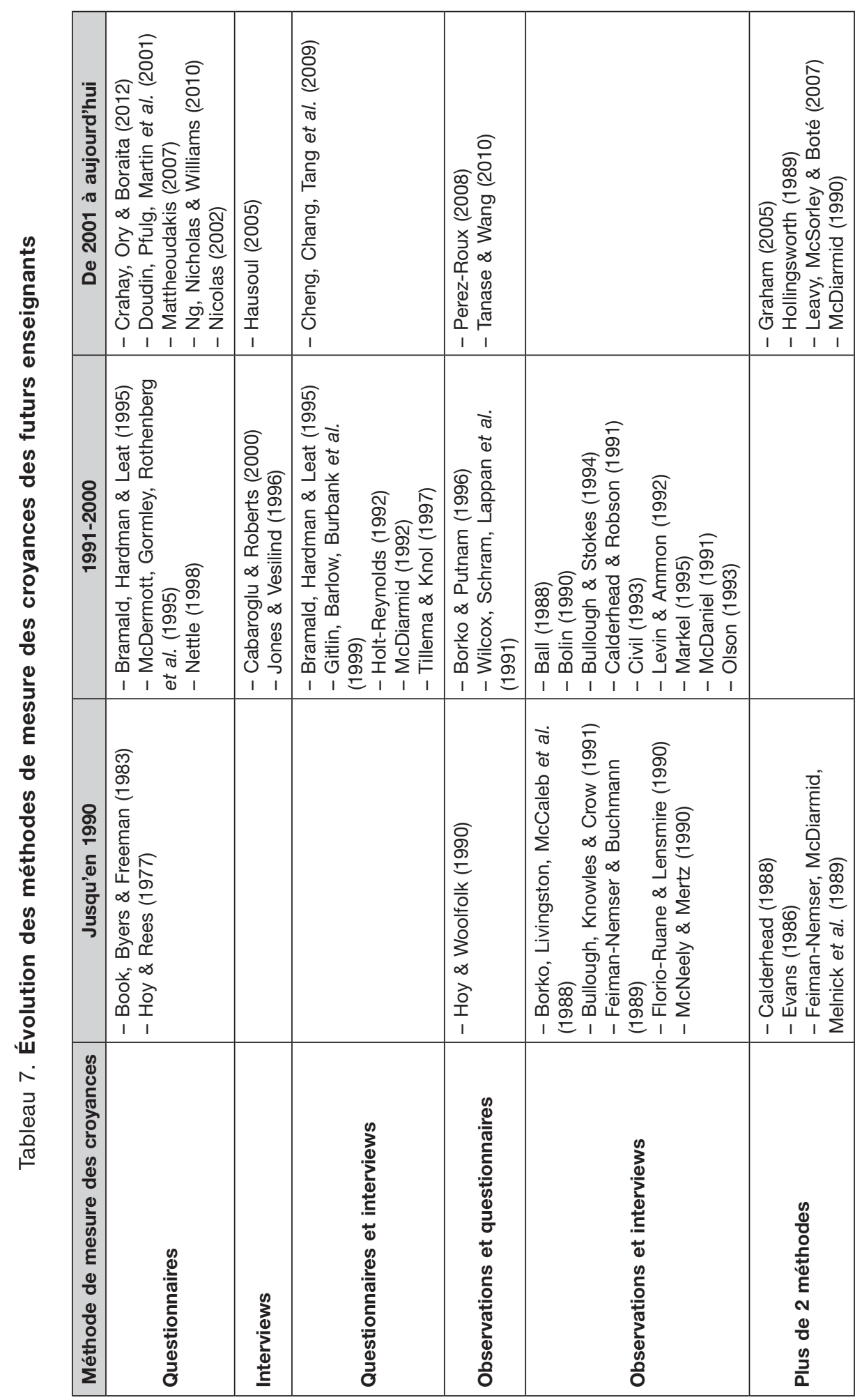



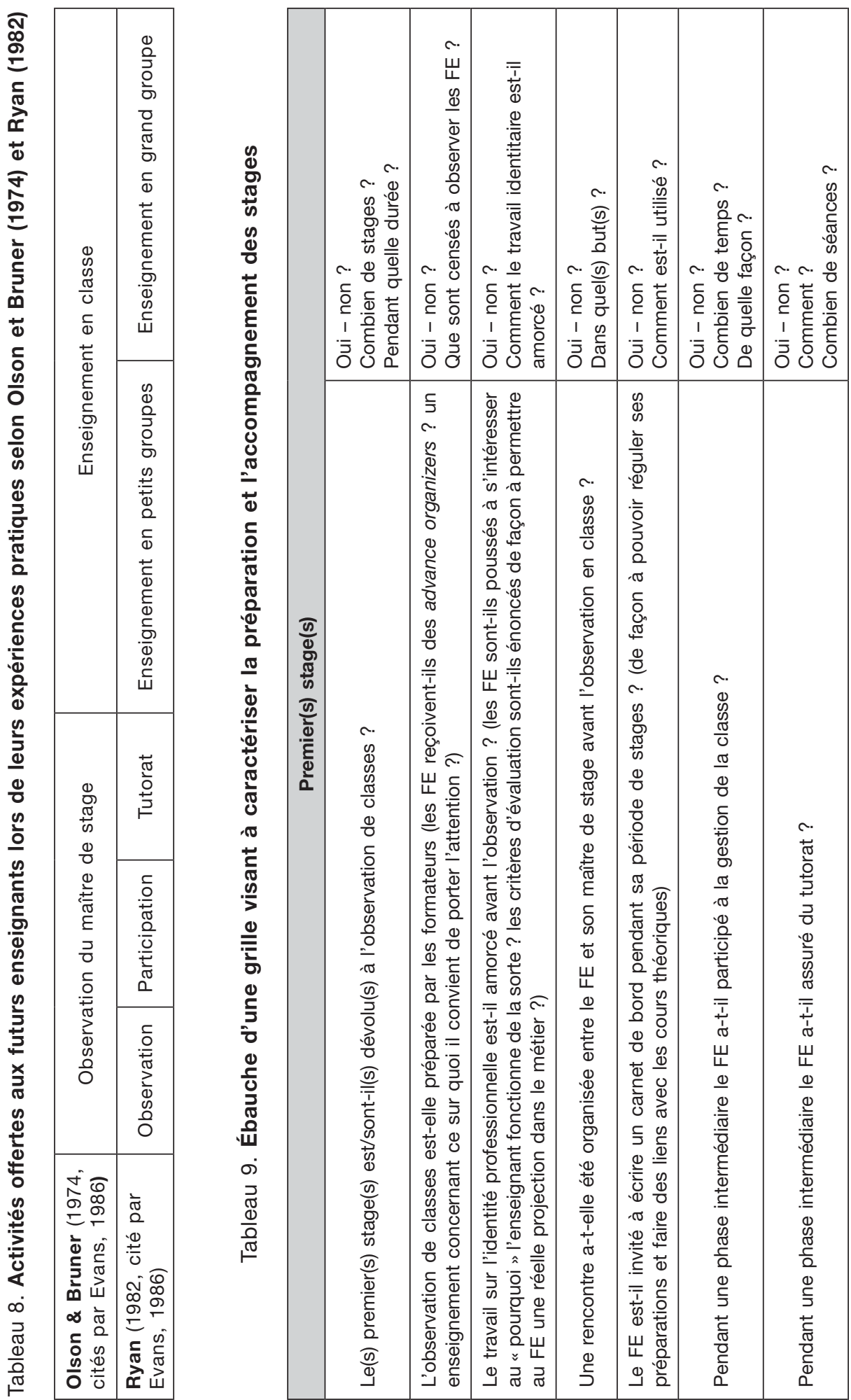


\begin{tabular}{|c|c|c|c|c|c|c|c|c|c|c|}
\hline 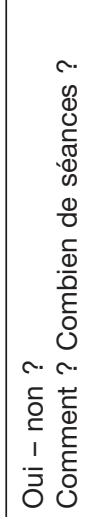 & 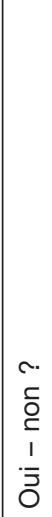 & 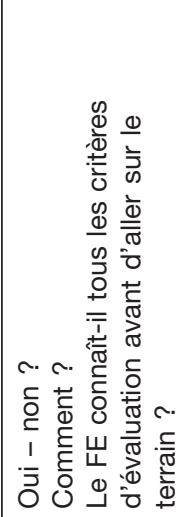 & & $\begin{array}{l}\text { r. } \\
\overline{0} \\
\bar{c} \\
1 \\
\bar{z} \\
\bar{c}\end{array}$ & 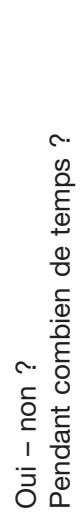 & 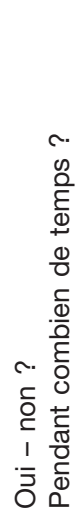 & $\begin{array}{l}\text {. } \\
\overline{0} \\
\check{0} \\
1 \\
\bar{c} \\
\bar{c}\end{array}$ & 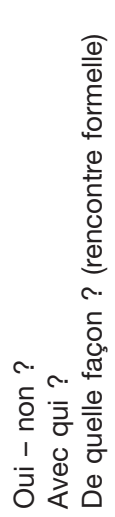 & 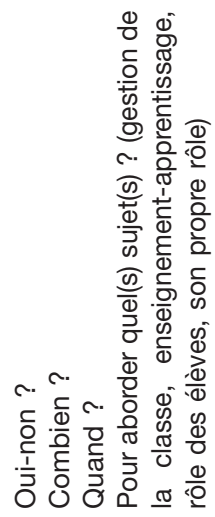 & 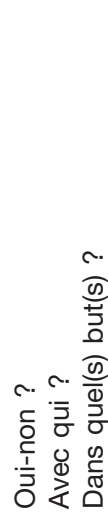 \\
\hline 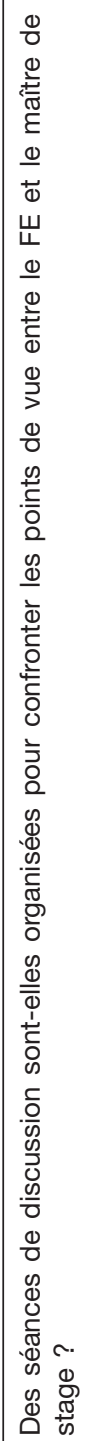 & 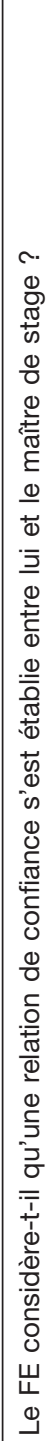 & 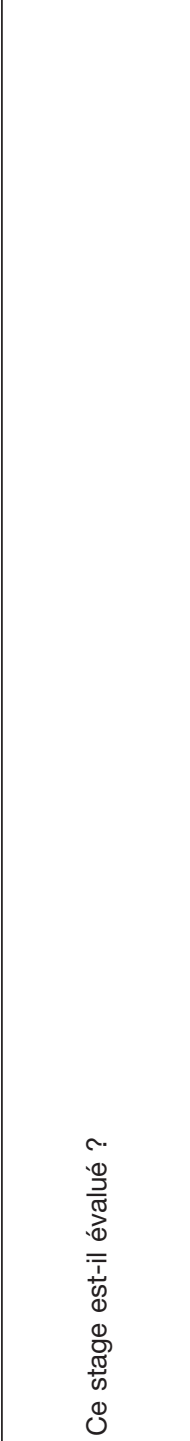 & 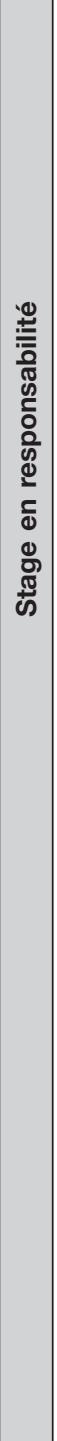 & 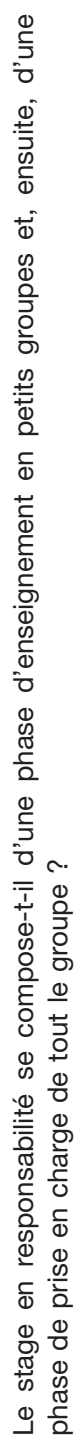 & 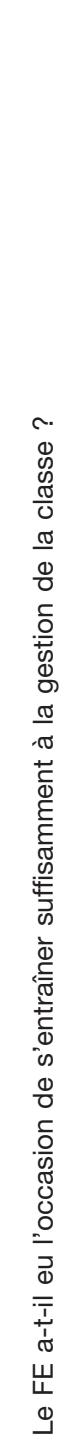 & 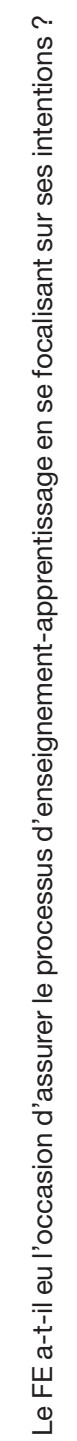 & $\begin{array}{l}0 \\
0 \\
0\end{array}$ & 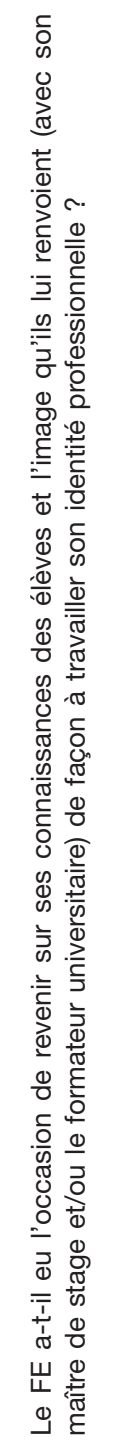 & 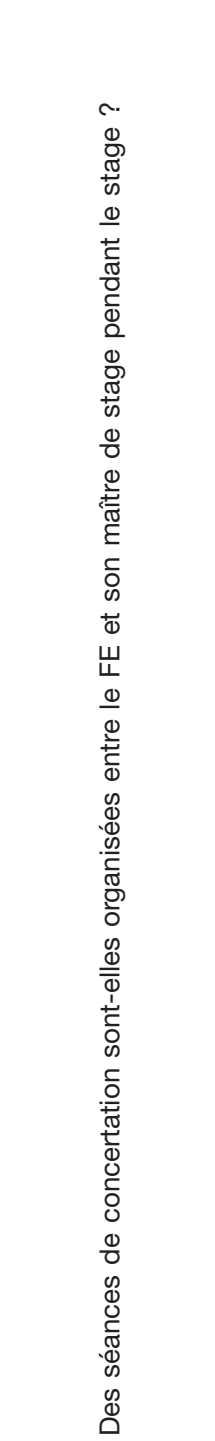 & 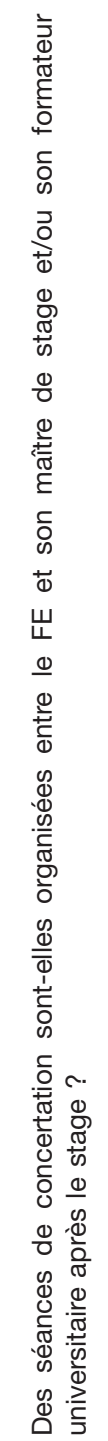 \\
\hline
\end{tabular}




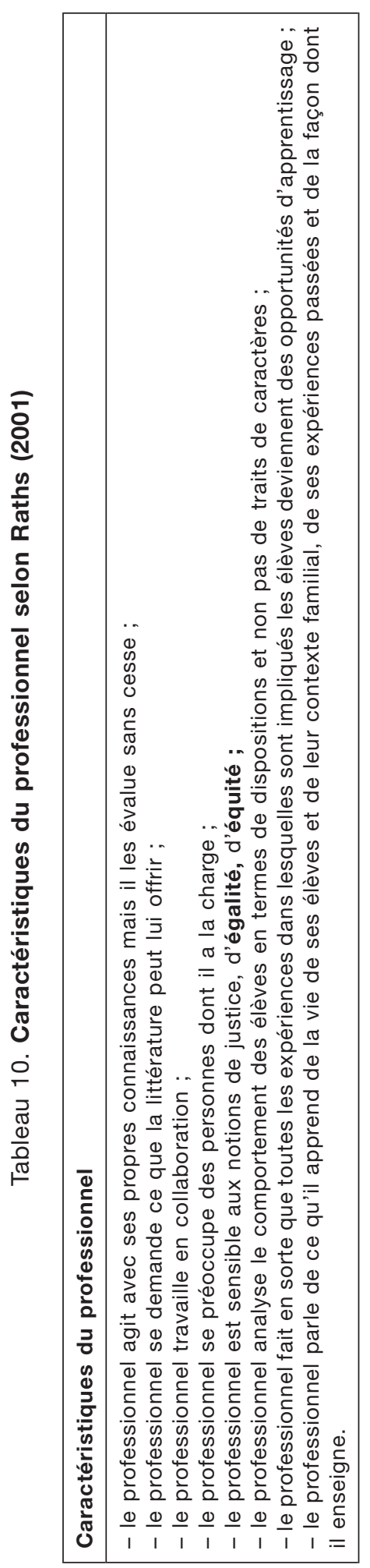

